\title{
PRACTICAL APPROACH TO BUILDING A MID-WAVE REMOTE SENSING SYSTEM
}

\section{By}

\author{
Benjamin Pyke
}

Copyright (C) Benjamin Pyke 2017

A Thesis Submitted to the Faculty of the

COLLEGE OF OPTICAL SCIENCES

In Partial Fulfillment of the Requirements

For the Degree of

MASTER OF SCIENCE

In the Graduate College

THE UNIVERSITY OF ARIZONA

2017 


\section{STATEMENT BY AUTHOR}

The thesis titled Practical Approach to building A Mid-Wave Remote Sensing System prepared by Benjamin Pyke has been submitted in partial fulfillment of requirements for a master's degree at the University of Arizona and is deposited in the University Library to be made available to borrowers under rules of the Library.

Brief quotations from this thesis are allowable without special permission, provided that an accurate acknowledgement of the source is made. Requests for permission for extended quotation from or reproduction of this manuscript in whole or in part may be granted by the head of the major department or the Dean of the Graduate College when in his or her judgment the proposed use of the material is in the interests of scholarship. In all other instances, however, permission must be obtained from the author.

SIGNED: Benjamin Pyke

APPROVAL BY THESIS DIRECTOR

This thesis has been approved on the date shown below:

Dr. John GreivenKamp

Date

Professor of Optical Sciences 


\section{Table of Contents}

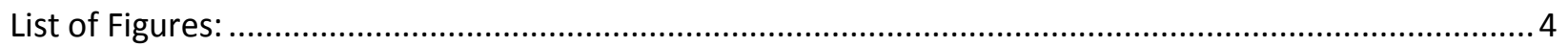

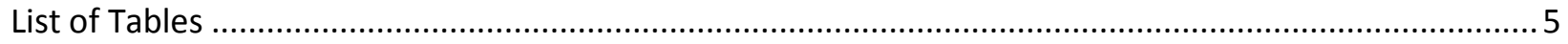

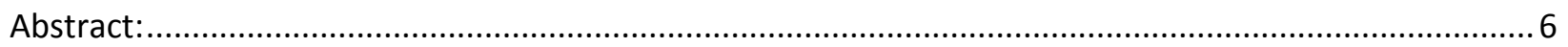

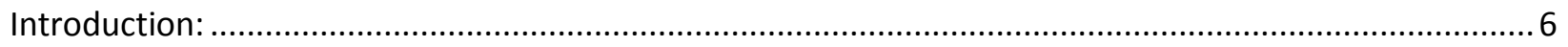

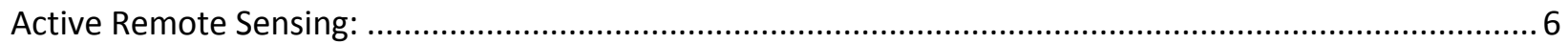

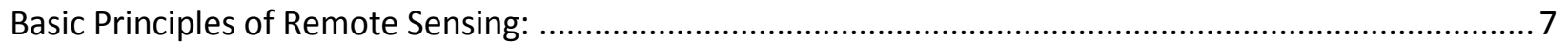

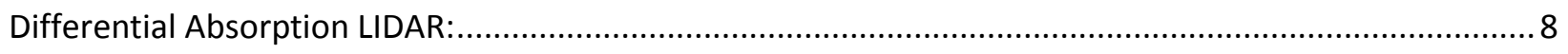

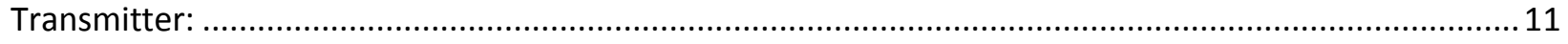

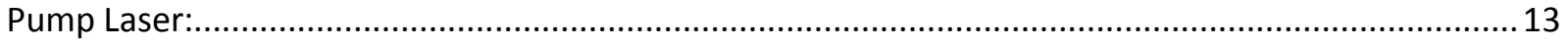

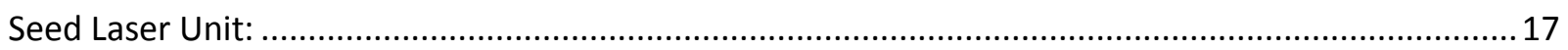

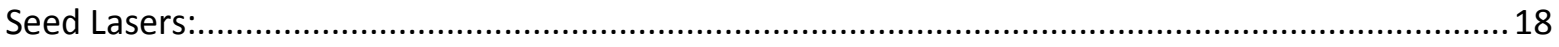

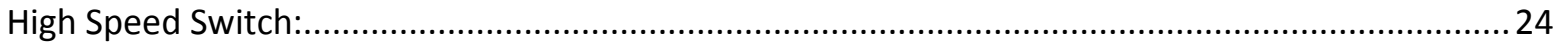

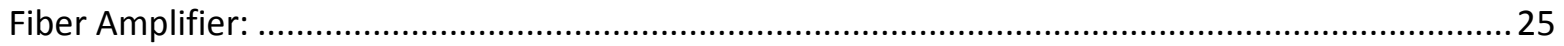

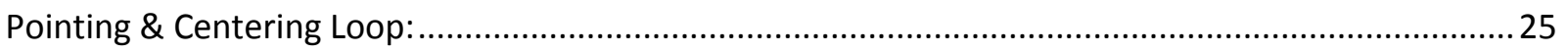

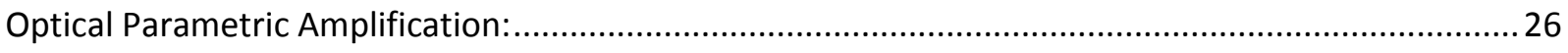

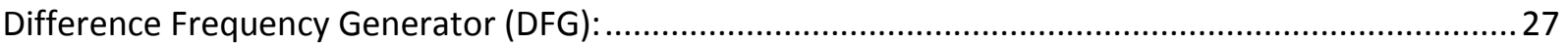

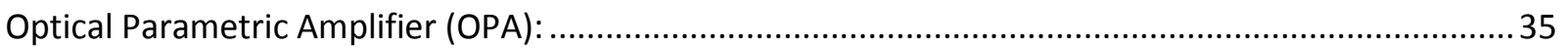

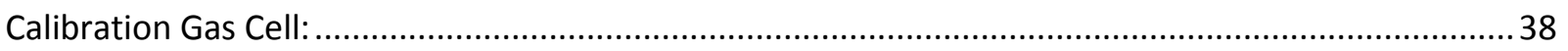

Receiver:

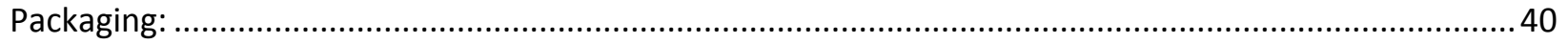

Control System:

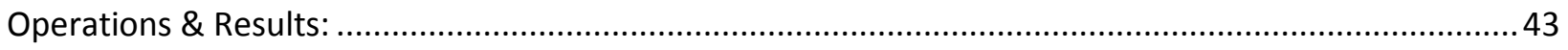

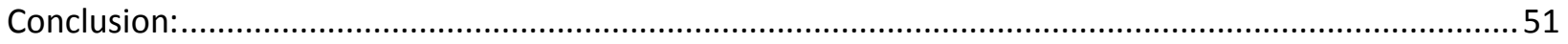

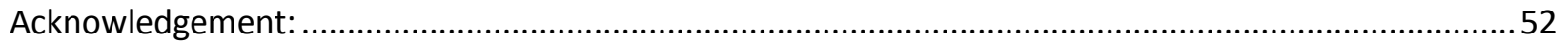

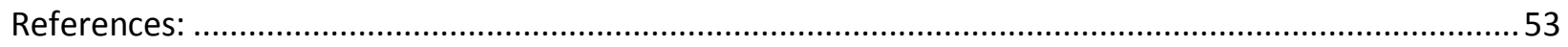




\section{List of Figures:}

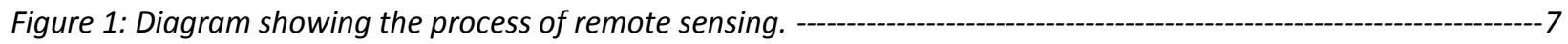

Figure 2: The absorption spectra of HCL from 3.2-3.5 microns. Data is from NIST ${ }^{(2)}$ and PNNL(3).-----

Figure 3: A sketch showing two possible scenarios of a CL measurement of 500ppm-m. -------------------9

Figure 4: Absorption spectra of both Butane and Isobutane. Data is from NIST ${ }^{(2)}$ and PNNL ${ }^{(3)}$. ----10

Figure 5: Block diagram of the elements in the transmitter.

Figure 6: A diagram of the different beam paths on the front $(A)$ side and the back $(B)$ side of the transmitter. ---- 12

Figure 7: Picture of the front side of the transmitter showing the main components. --------- 12

Figure 8: Picture of the back side of the transmitter showing the main components. ------------------13

Figure 9: The sketch of the oscillator for the pump laser. -

Figure 10: The beam shape \& size along with the output specifications of the oscillator. ------- 14

Figure 11: The oscillator linewidth measurement with a 50\%, $1.5 \mathrm{~mm}$ etalon in the cavity. ----------15

Figure 12: A sketch of the layout of three amplifiers in the MOPA. ---------------------15

Figure 13: The beam shape and size along with the output specifications of the MOPA. ----------16

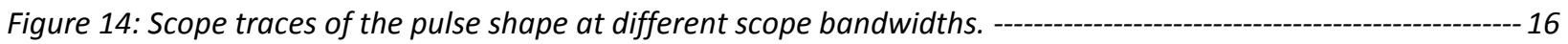

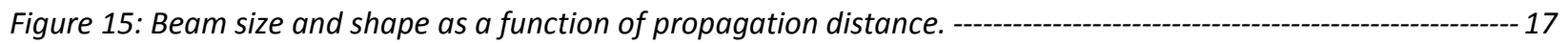

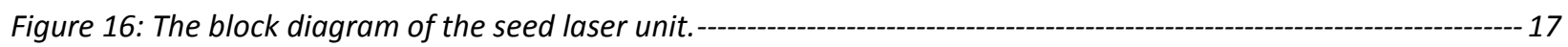

Figure 17: Picture of the Pirelli seed diode mounted with the LLNL designed control boards. --------18

Figure 18: Diode tuning data from the manufacturer. (Little $\left.{ }^{(4)}\right)$ -

Figure 19: Measurement of the wavelength as a function of tuning mirror voltage. ---------- 20

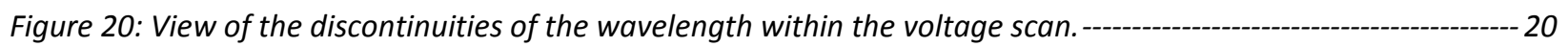

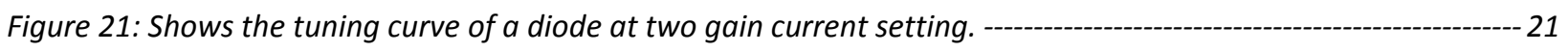

Figure 22: Variation of step center wavelength with current and curve fit with a simple relation to Igain2 (Little $\left.{ }^{(4)}\right)$

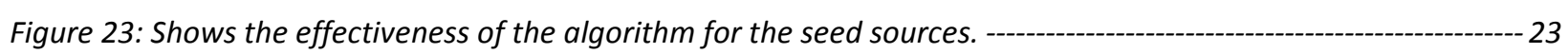

Figure 24: The picture and schematic of the EOSpace 1x8 switch. ----------------------------------------------------------24

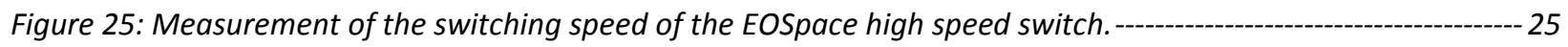

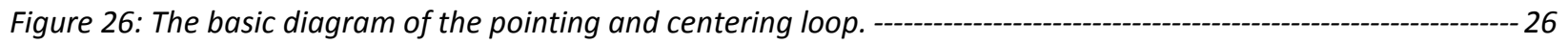

Figure 27: Diagram of the difference frequency generation technique used. ------------------------------------------- 27

Figure 28: Graph showing how the poling period changes as a function of the idler wavelength with the crystal at room temperature and at $180^{\circ} \mathrm{C}$.

Figure 29: The external input angle of the pump and signal beam to phase-match with a poling period of 29.3

microns.

Figure 30: Sketch of the PPLN crystal used in the DFG.

Figure 31: The external input angle of the pump and signal beam to phase-match with a poling period of 23.9

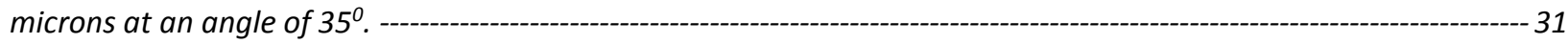

Figure 32: Shows the split ratio of the pump power to the DFG and OPA. -------------------------------- 32

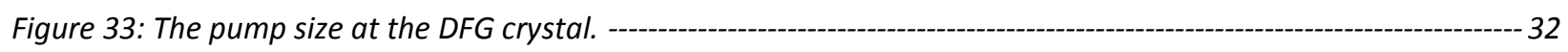

Figure 34: The plot of the single pass gain of the DFG as a function of crystal length. -------------------- 33

Figure 35: The power out of the DFG as a function of the pump power with a $50 \mathrm{~mm}$ long crystal. -------------------- 33

Figure 36: Temporal measurements of the galvo motors. -

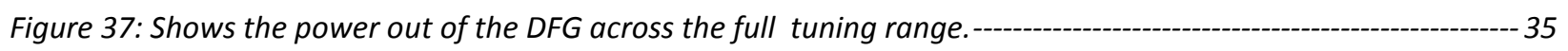

Figure 38: The diagram of the amplification process used. --------------------------------------------------------- 35

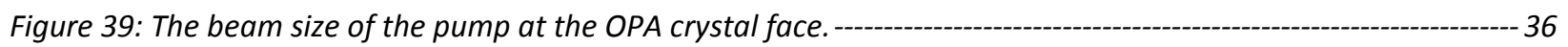

Figure 40: The gain calculation of the OPA as a function of crystal length. 
Figure 41: The power out of the OPA as a function of pump power.

Figure 42: Shows the power out of the OPA (at low pump power) and the galvo 2 counts over the full tuning range.

Figure 43: (a) Picture of the Coherent software used to get the galvo 3 (b) settings. -------------- 38

Figure 44: A diagram of the calibration gas cell system.

Figure 45: The calibration gas cell fit to $\mathrm{HCl}$ absorption peak. ------------------------------------------------------ 39

Figure 46: $A$ sketch of the basic concept of the receiver. ---------------------------- 40

Figure 47: The mechanical layout of the ground based remote sensing system. ------- 41

Figure 48: The Zemax model of the launch and receive optics. ------------------------------- 42

Figure 49: The first image of the transmitted beam collected by the receiver. --------------------------- 42

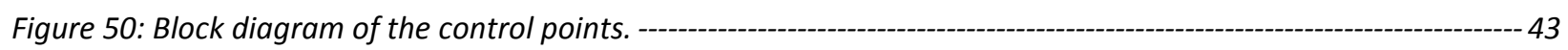

Figure 51: The absorption spectrum of butane with the test locations. ------------ 44

Figure 52: The program used to create the Line Sequencing Description and Table. ------------------------------------- 44

Figure 53: Plot showing two complete LSDs of the photodiode which indicate the flagged pulses and the source that

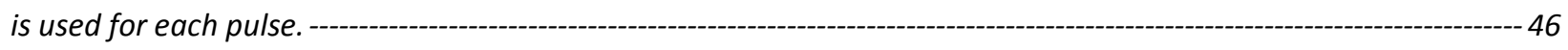

Figure 54: The average returns for each source over an entire $L S D$. ----------------------------------------------------------- 46

Figure 55: The spatial and temporal averaged amplitude for each of the test wavelengths. ------------ 47

Figure 56: The averaged return for each source when butane gas is present. ----------------------------------- 48

Figure 57: The concentration path-length calculation averaged both spatially and temporally. ---------------48

Figure 58: A plot showing the absorption of butane along with the atmospheric transmission with the test locations

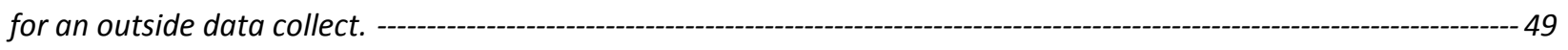

Figure 59: Diagram of the outdoor data collect. --------------------------------------------------------------------49

Figure 60: The spatial and temporal averaged amplitude for each of the sampled wavelengths. -------------- 50

Figure 61: The concentration path-length calculation of butane for the second example. ------------------ 50

\section{List of Tables}

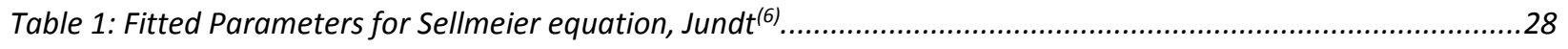

Table 2: The absorption of butane at the location of the test wavelengths. .......................................................47 


\begin{abstract}
:
The purpose of this project, Laser Active Transmitter \& Receiver (LATR), was to build a mobile ground based remote sensing system that can detect, identify and quantify a specific gaseous species using Differential Absorption LIDAR (DIAL). This thesis project is concerned with the development and field testing of a mid-wave infrared active remote sensing system, capable of identifying and quantifying emissions in the 3.2-3.5 micron range. The goal is to give a brief description of what remote sensing is about and the specific technique used to analyze the collected data. The thesis will discuss the transmitter and the associated subsystems used to create the required wavelength, and the receiver used to collect the returns. And finally, the thesis will discuss the process of collecting the data and some of the results from field and lab collections.
\end{abstract}

\title{
Introduction:
}

What is remote sensing? Remote sensing is the science of obtaining information about an object or feature without physically coming in contact with the object or feature. The human body is a good example of a remote sensing instrument. It is equipped with multiple sensors that are used to collect data remotely; 1) our eyes through reflected visible light, 2) our ears through mechanical vibrations, 3 ) our nose through smelling aromas, and 4) our skin has the ability to detect radiated heat. There are two classifications of remote sensing sensors; passive and active. Passive devices detect natural (the sun's) energy that is reflected, emitted or absorbed from the object of interest. Passive systems are attractive due to the inherent broad area coverage as well as the relatively simple equipment requirement (only a receiver). In an active remote sensing system, the energy is generated and transmitted from the sensors platform to the object. The energy is reflected back from the object and recorded using the sensor's on the remote sensing platform. A simple example of passive remote sensing is taking a picture with a standard camera, where an active remote sensing example would be taking a picture with a camera using the built-in flash. It is generally agreed upon that if both a passive and active sensor could perform the task to the required sensitivity, the passive system is usually the preferred choice.

\section{Active Remote Sensing:}

An active sensor emits radiation which is directed toward the object or area to be investigated. The radiation that is reflected from the object is detected and measured by the sensor. Some advantages for active sensors are:

- The ability to obtain measurements anytime, regardless of the time of day, season, or weather.

- To examine wavelengths that are not sufficiently provided by the sun

- To better control the way that the object or area is illuminated.

Some disadvantages are: 
- It requires the generation of a large amount of energy to adequately illuminate the object or area, which generally means the instrument is larger, heavier, and requires more energy to power it.

- In most cases the transmitter/receiver must be scanned to cover an equivalent area.

- In general, the cost is higher because the instrument is both a transmitter and a receiver.

\section{Basic Principles of Remote Sensing:}

Detection and discrimination of objects or materials means detecting and recording the radiant energy that is reflected or emitted by the target. Different objects reflect, absorb, or emit different amounts of energy in different bands of the electromagnetic spectrum. The amount of energy that is reflected, absorbed, or emitted depends on the characteristics of both the material (structural, chemical, and physical) and the incident energy (incident angle, intensity, and wavelength). There are a number of stages in the process of remote sensing. The main stages are the following:

1. Emission of electromagnetic radiation

2. Propagation of energy from the source to the target

3. Interaction of the electromagnetic radiation with the target (reflection, absorption, emission)

4. Propagation of the energy from the target to the sensor

5. Recording of the energy by the sensor

6. Processing the data

7. Analysis of the data

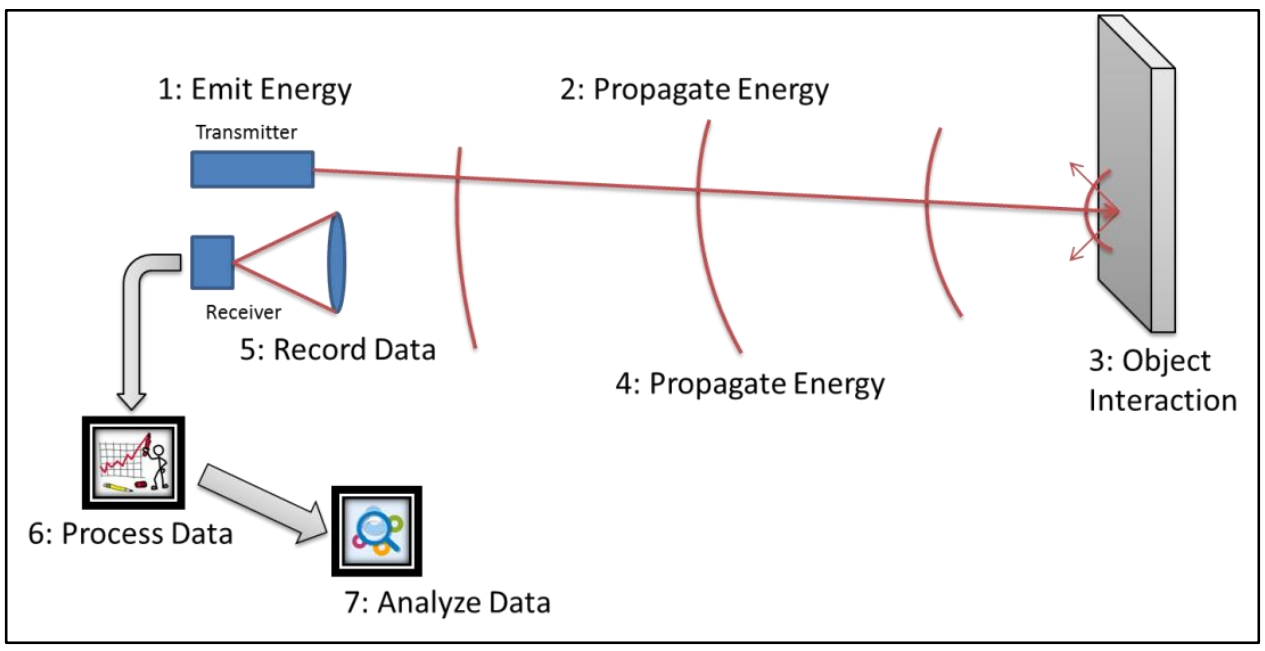

Figure 1: Diagram showing the process of remote sensing. 


\section{Differential Absorption LIDAR:}

This system processes the data using the Differential Absorption LIDAR (DIAL) technique to determine if a target material, gas in this case, is present or not, and to what concentration. This technique uses two or more wavelengths, depending on the target material, and compares the return of the first wavelength with the return of the second. The idea is to have one wavelength tuned to an absorption feature (On Wavelength) of the target material and the other wavelength tuned away from the absorption feature. For example, hydrogen chloride $(\mathrm{HCl})$ has many unique narrowband absorption features in the Mid-Wave Infrared (MWIR) range of this system. The absorption features for $\mathrm{HCl}$ are shown in Figure 2 below.

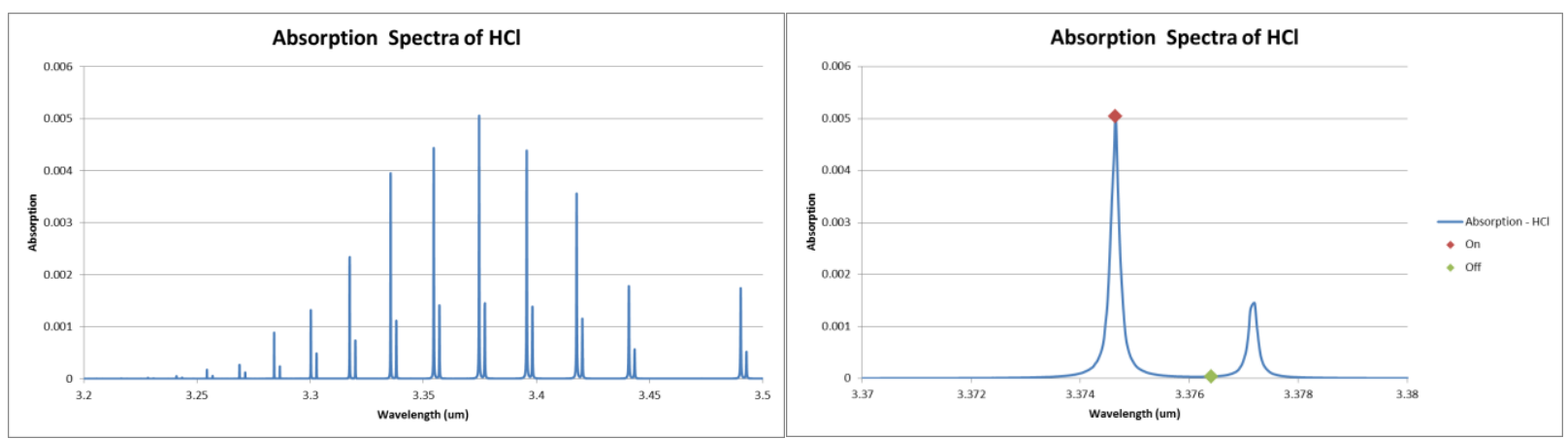

Figure 2: The absorption spectra of HCL from 3.2-3.5 microns. Data is from NIST ${ }^{(2)}$ and PNNL(3).

If two narrowband laser wavelengths are emitted from the transmitter, one centered on the $\mathrm{HCl}$ absorption feature (red diamond at 3.3746um, Figure $2 \mathrm{~b}$ ) and the other close-by but off the absorption feature (green diamond at 3.3764um, Figure $2 \mathrm{~b}$ ) then this two line DIAL system would be able to detect the presence of $\mathrm{HCL}$.

In the above example with a single material and two wavelengths, the equation to calculate the concentration-path length is:

$$
C L=\frac{1}{2\left(\alpha_{1}-\alpha_{2}\right)} \ln \left(\frac{\rho_{2}}{\rho_{1}}\right)
$$

where $\alpha_{i}$ is the absorptivity of the material at wavelength $\lambda_{i}$ and $\rho_{i}$ is the LIDAR return at wavelength $\lambda_{i}$. So what is concentration-path length (CL)? Gas concentration is measured in parts per million (ppm) by volume. If a space measuring one million cubic meters has 50 cubic meters of air replaced by a gas, then the gas concentration in that space is said to be $50 \mathrm{ppm}$. Concentration-path length is the measurement of the total amount of a specific gas in the path of the laser beam between the transmitter and the receiver. 

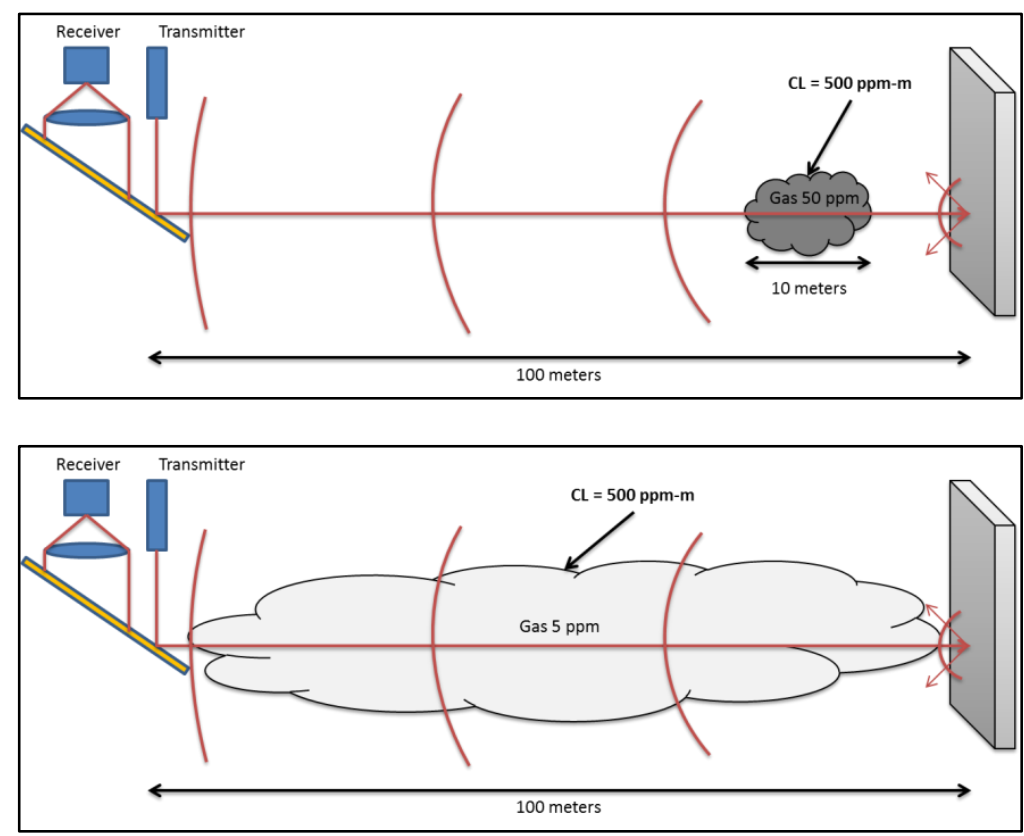

Figure 3: A sketch showing two possible scenarios of a CL measurement of 500ppm-m.

Since $\mathrm{HCl}$ has unique narrow absorption features, a two line DIAL system is sufficient to detect the presence of the material. But what happens if the absorption feature of the target material is broad and similar to other materials? For example, butane and iso-butane (the absorption spectra of both materials) shown in Figure 4, are similar except for some very sharp features. In order to distinguish between these materials, more wavelengths are needed to be emitted from the transmitter. The more wavelengths that are emitted from the transmitter, the easier it is to distinguish between the materials. By placing the selected wavelengths on a specific absorption feature, the material of interest can be detected and identified. 


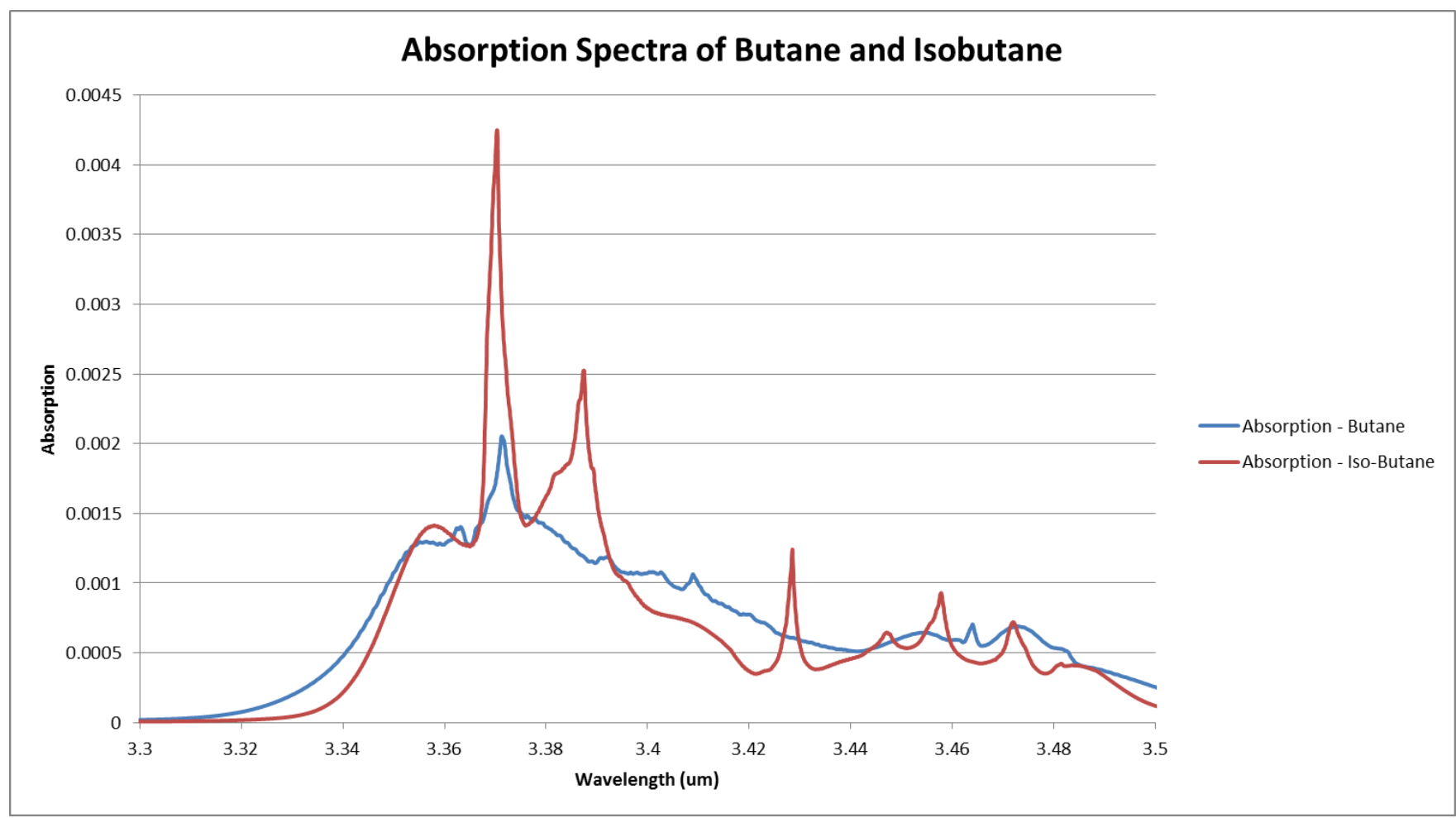

Figure 4: Absorption spectra of both Butane and Isobutane. Data is from NIST ${ }^{(2)}$ and PNNL(3).

The calculation for the concentration-path length is a little more complicated when more than two wavelengths are used. As defined by Warren ${ }^{(1)}$, the observation vector is

$$
\vec{S}_{i}=\frac{1}{2} \ln \left(\frac{\rho_{i+1}}{\rho_{i}}\right)
$$

where $i$ is the wavelength lines used. The absorption matrix $\mathrm{K}$ is defined by

$$
K_{i, l}=\alpha_{i}^{l}-\alpha_{i+1}^{l}
$$

where $\alpha_{i}^{l}$ is the absorption of material / at wavelength $\lambda_{i}$. The concentration-path length is then calculated by

$$
C L=\frac{K^{T} \vec{s}}{K^{T} \mathrm{~K}}
$$

Another criterion when selecting the lines that must be taken into consideration is the atmospheric transmission of each particular wavelength. The selected wavelengths must avoid the absorption of the water vapor lines, which will be discussed later. 


\section{Transmitter:}

This project reactivated and upgraded a Mid-Wave Infrared (MWIR) transmitter that was previously developed by Lawrence Livermore National Lab (LLNL), and integrated it and the co-located optical receiver into a mobile trailer system. The transmitter uses multi-element laser systems to achieve the desired wavelengths necessary to detect the target material. It consists of the following elements:

1. The Master Oscillator Power Amplifier (MOPA) that was designed and built by LLNL. The MOPA is roughly a 100 Watt, neodymium-doped vanadate pump laser running at a rep-rate of $10 \mathrm{kHz}$.

2. The Seed Laser Unit contains six tunable C-band external cavity laser diodes and an Erbiumdoped fiber amplifier that can produce an output up to 2 watts.

3. The pointing and centering loop uses two USB CCD cameras and two motorized mirror mounts to keep the pump laser aligned.

4. The splitter splits the pump light so that approximately $1 / 4$ the power goes to pump the DFG and $3 / 4$ goes to pump the OPA.

5. The Difference Frequency Generation (DFG) is a non-linear optical process where the generation of the mid-wave frequency is the difference between the pump and seed frequencies.

6. The Optical Parametric Amplifier (OPA) amplifies the light that is generated in the DFG by a non-linear optical process.

7. The expansion telescope expands the size of the beam by roughly 15 times before the light is transmitted to the target.

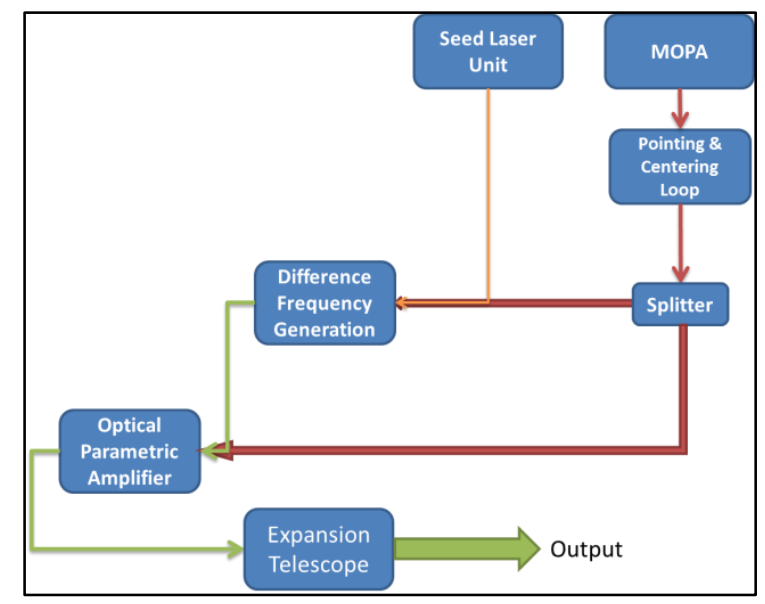

Figure 5: Block diagram of the elements in the transmitter. 


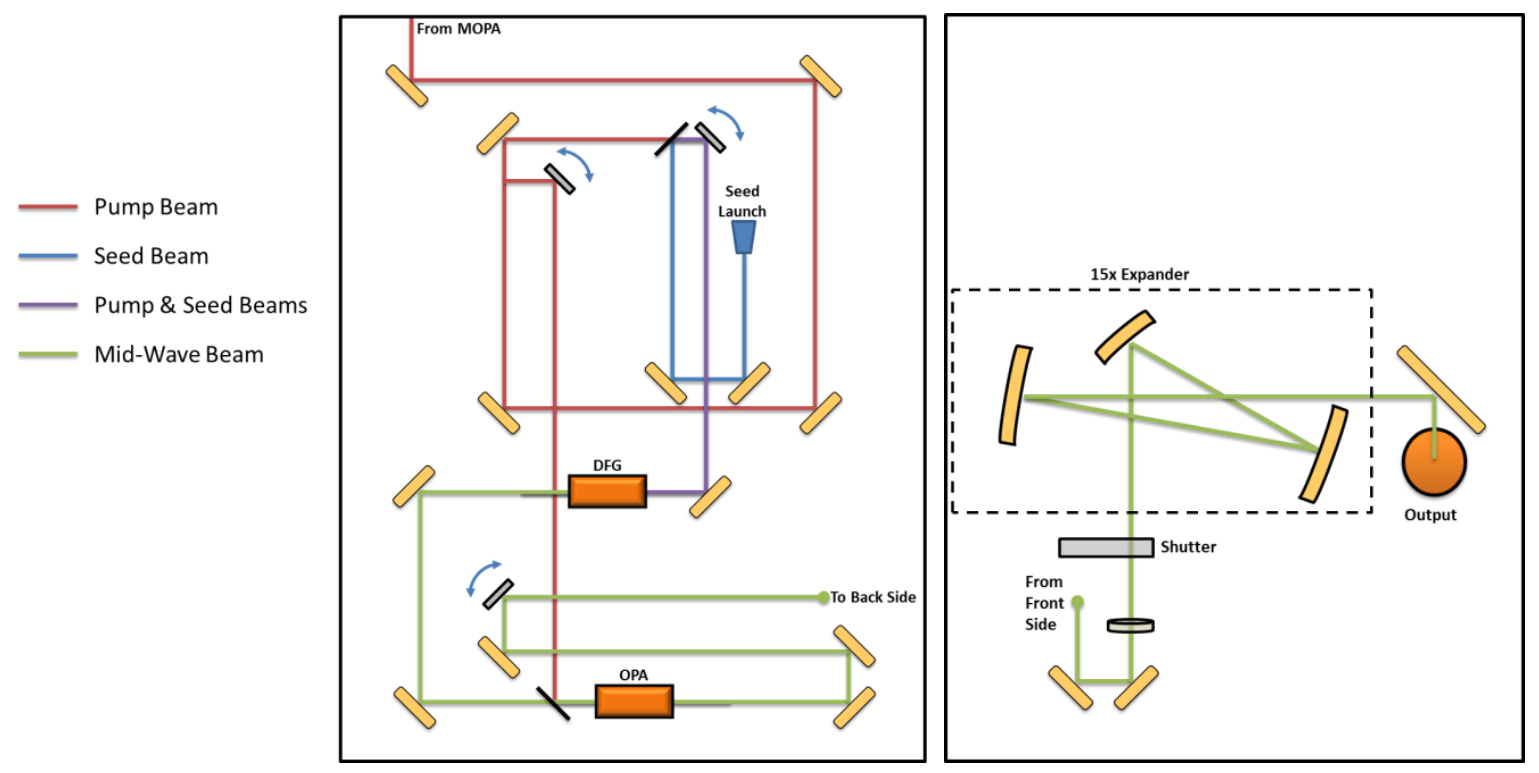

Figure 6: A diagram of the different beam paths on the front (A) side and the back (B) side of the transmitter.

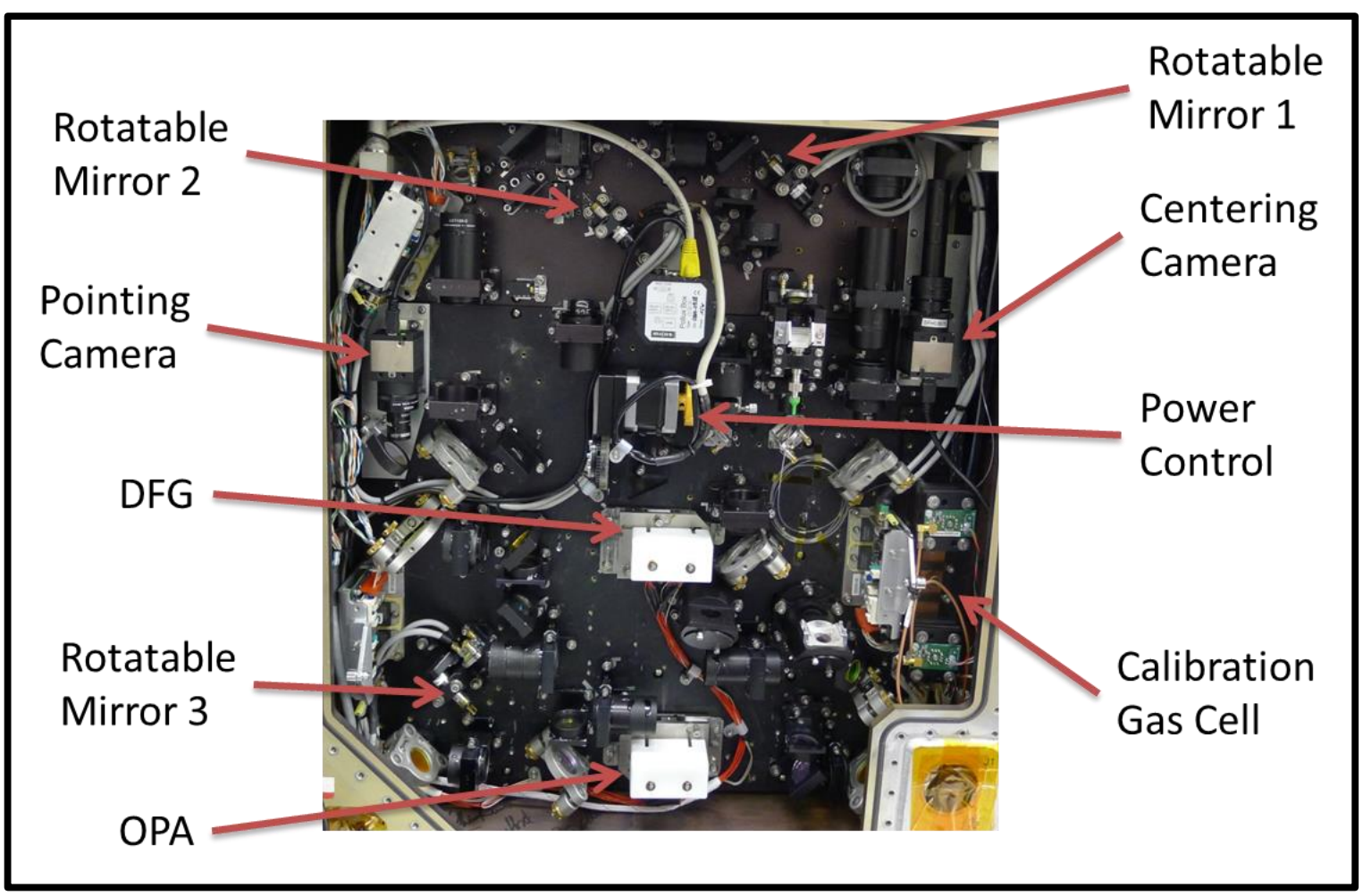

Figure 7: Picture of the front side of the transmitter showing the main components. 


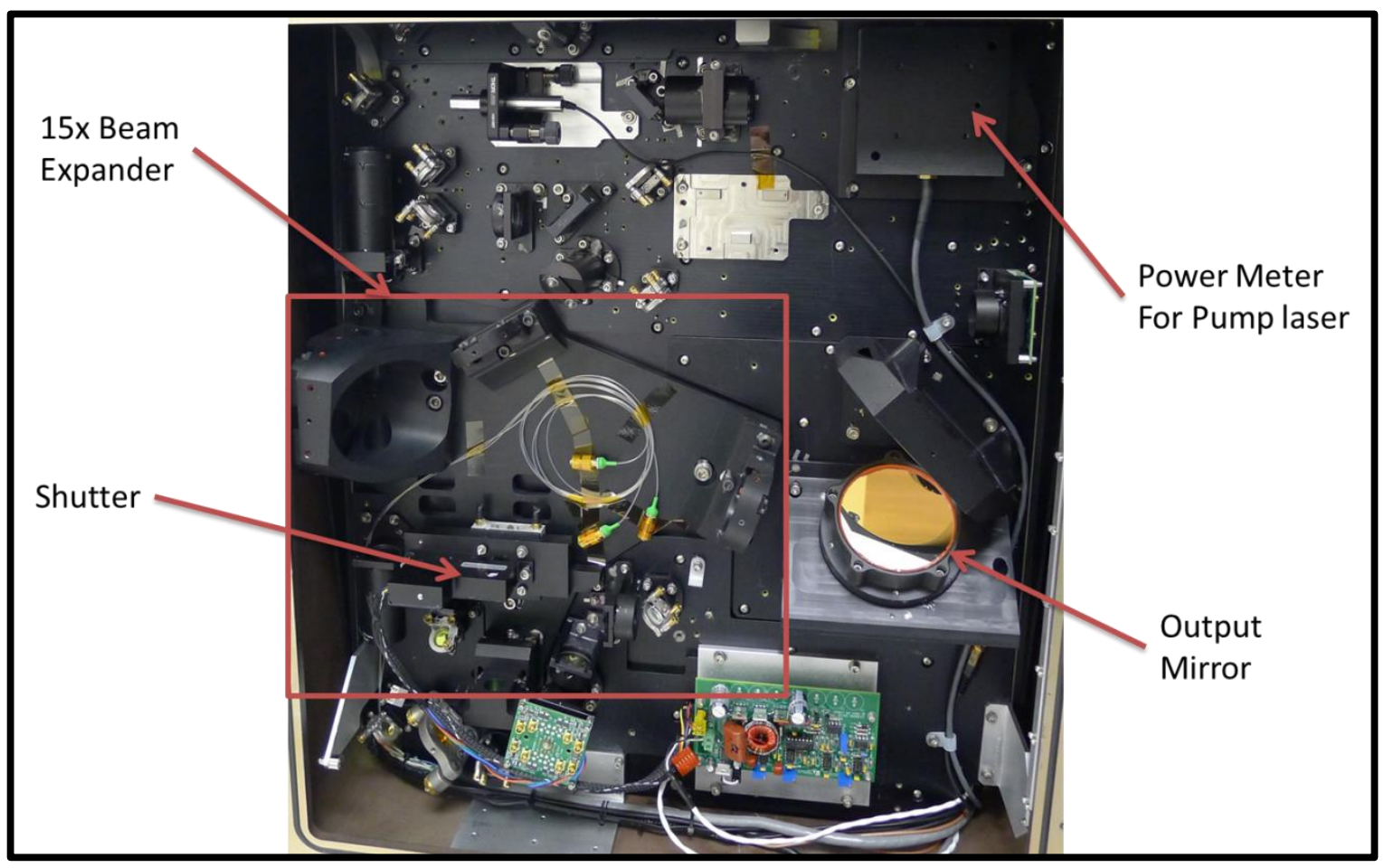

Figure 8: Picture of the back side of the transmitter showing the main components.

\section{Pump Laser:}

The pump laser is in a Master Oscillator Power Amplifier (MOPA) configuration which consists of a Qswitched oscillator and 12 single pass amplifiers. The term Master Oscillator Power Amplifier refers to the configuration consisting of a master laser (or seed laser) and an optical amplifier in order to boost the output power. Although this configuration can be more complex than a laser which produces the required output power, the MOPA concept has some advantages ${ }^{(5)}$. First, with a MOPA it can be easier to reach the required performance specifications in parameters like linewidth, wavelength, beam quality and pulsewidth, if the required output power is very high. This is true since the laser parameters are decoupled from the generation of high powers. Secondly, this configuration allows the use an etalon to tune the pump wavelength in the relatively low intensity of the oscillator cavity. Finally, the optical intensities are lower in the amplifier compared with the inter-cavity intensities in a laser.

The oscillator in our MOPA design (shown below) is configured in a $Z$ cavity with the laser rod pumped through one end. The laser rod is a $4 \times 4 \times 27 \mathrm{~mm}$ neodymium-doped vanadate crystal which is pumped by a Coherent 50 watt, 808nm fiber-coupled laser diode (FAP). 


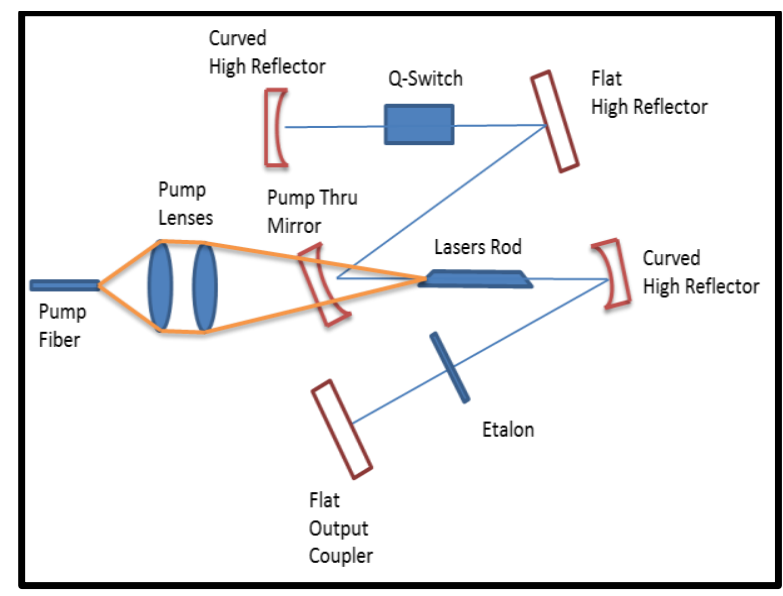

Figure 9: The sketch of the oscillator for the pump laser.

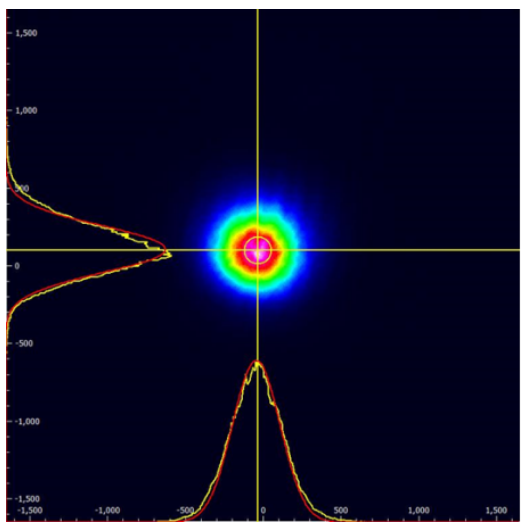

- Output=5.1W @ 46A \& $15^{\circ} \mathrm{C}$

- Pulsewidth 16.3ns (1GHz)

- Beamsize @ Output Coupler

- Effective diameter $=0.68 \mathrm{~mm}$

- Gaussian X $=0.64 \mathrm{~mm}$

- Gaussian $Y=0.63 \mathrm{~mm}$

$-X / Y=101 \%$

Figure 10: The beam shape $\&$ size along with the output specifications of the oscillator. 


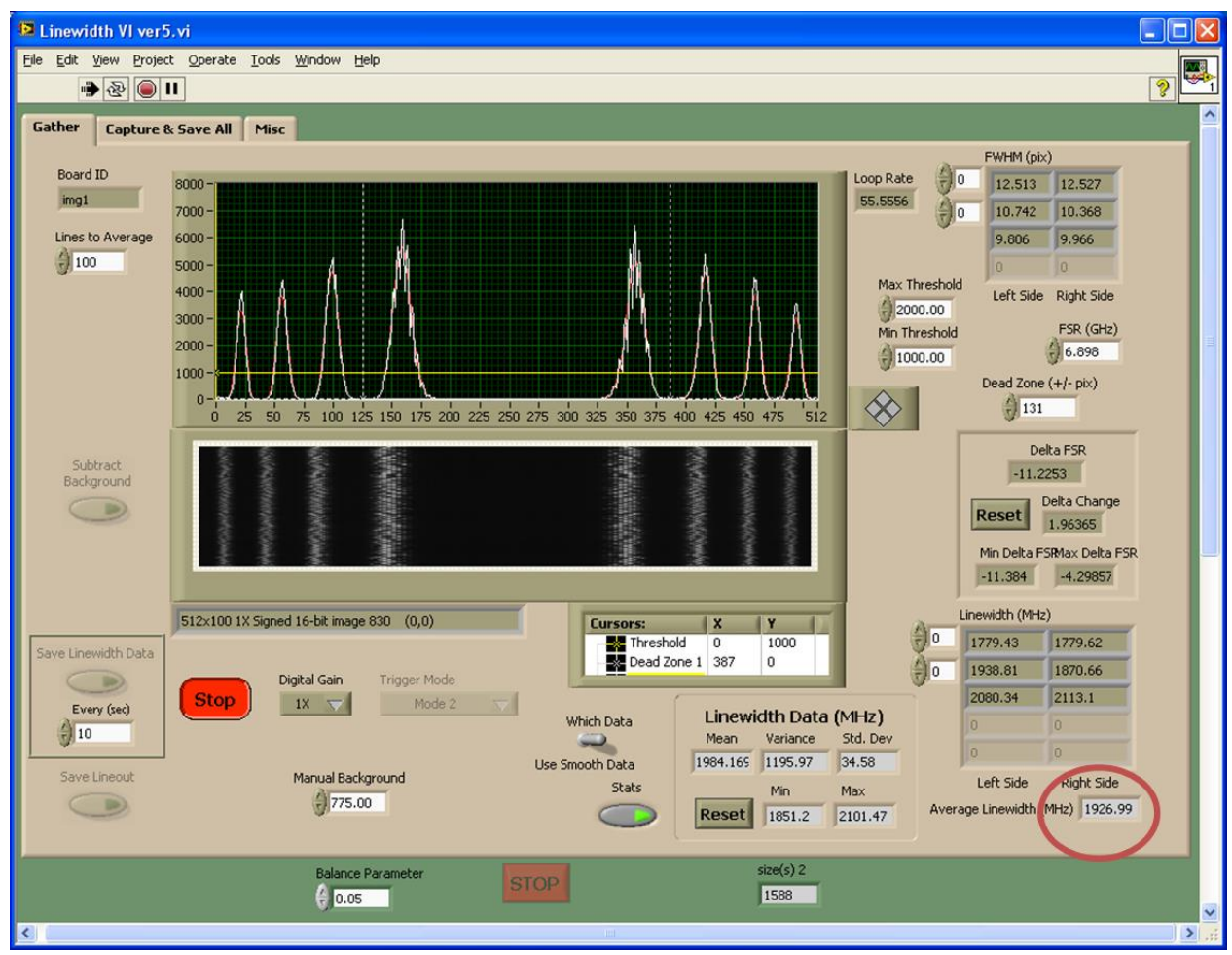

Figure 11: The oscillator linewidth measurement with a $50 \%, 1.5 \mathrm{~mm}$ etalon in the cavity.

The power amplifier in the MOPA design consists of 12 single pass, end pumped vanadate amplifiers. Each amplifier is pumped by a 50 watt Coherent fiber-coupler laser diode that has been individually temperature-tuned to $808 \mathrm{~nm}$. The MOPA "box" is a two sided design with the cooling channels for each rod in the center. The oscillator and the first four amplifiers are on one side while the final eight amplifiers are on the other side.

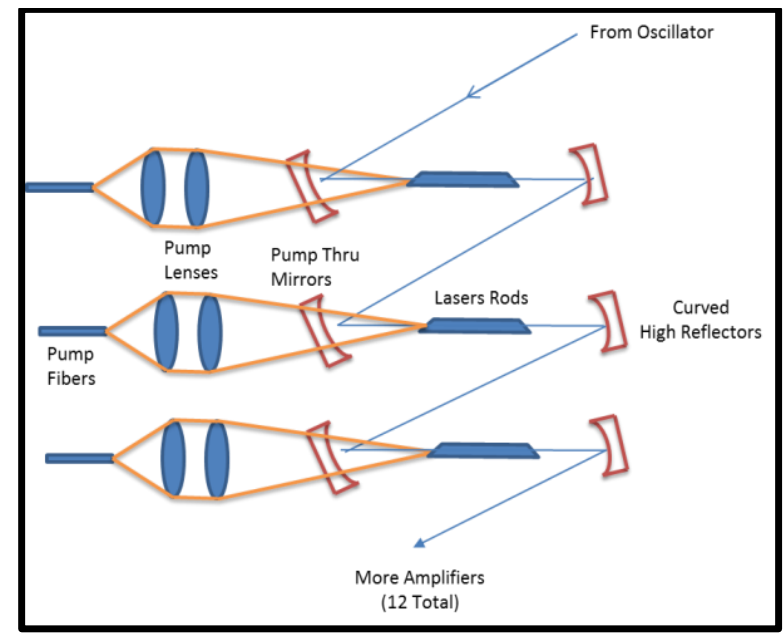

Figure 12: A sketch of the layout of three amplifiers in the MOPA. 


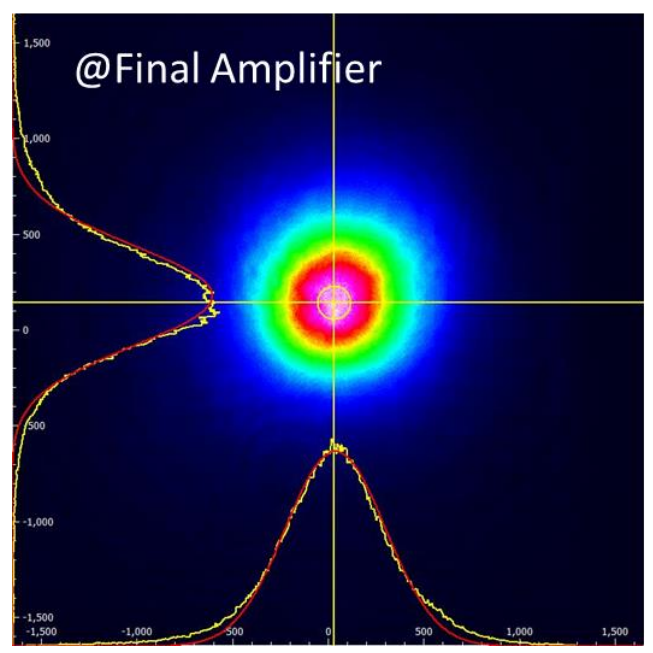

- Output $=108.3 \mathrm{~W}$

- Pulsewidth 20.5ns (1GhZ)

- Output Beamsize

- Effective diameter $=1.21 \mathrm{~mm}$

- Gaussian X $=1.09 \mathrm{~mm}$

- Gaussian $Y=1.09 \mathrm{~mm}$

- $X / Y=100 \%$

- $\quad$ Far field $=91.6 \mathrm{~W} / 109.2 \mathrm{~W}=83.9 \%$

Figure 13: The beam shape and size along with the output specifications of the MOPA.

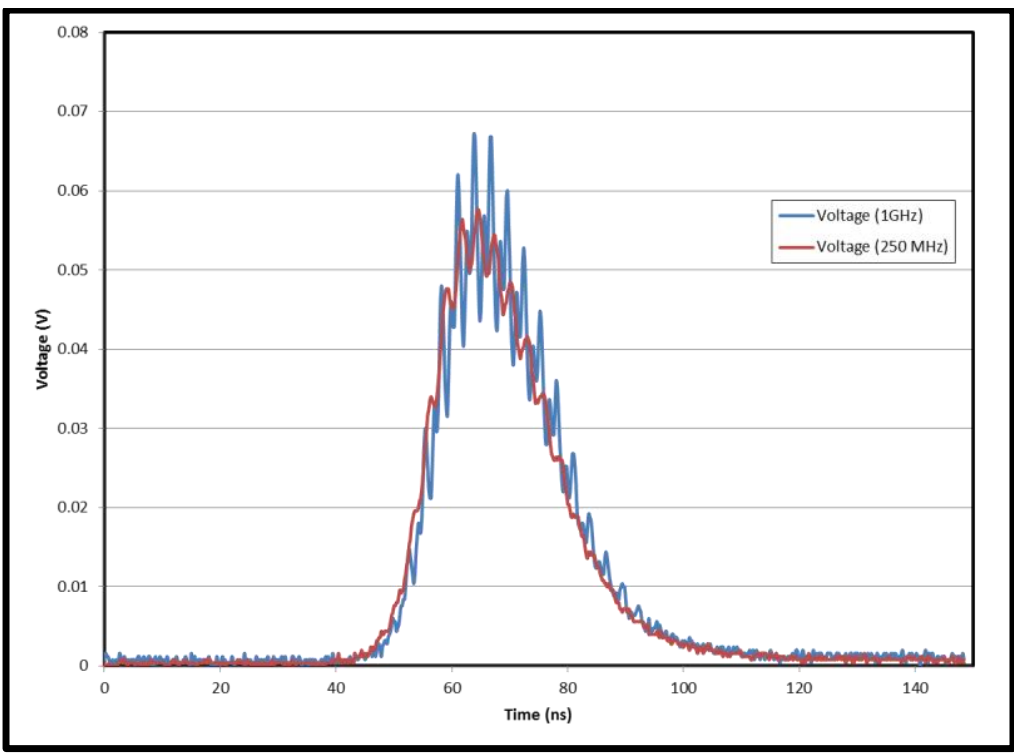

Figure 14: Scope traces of the pulse shape at different scope bandwidths. 

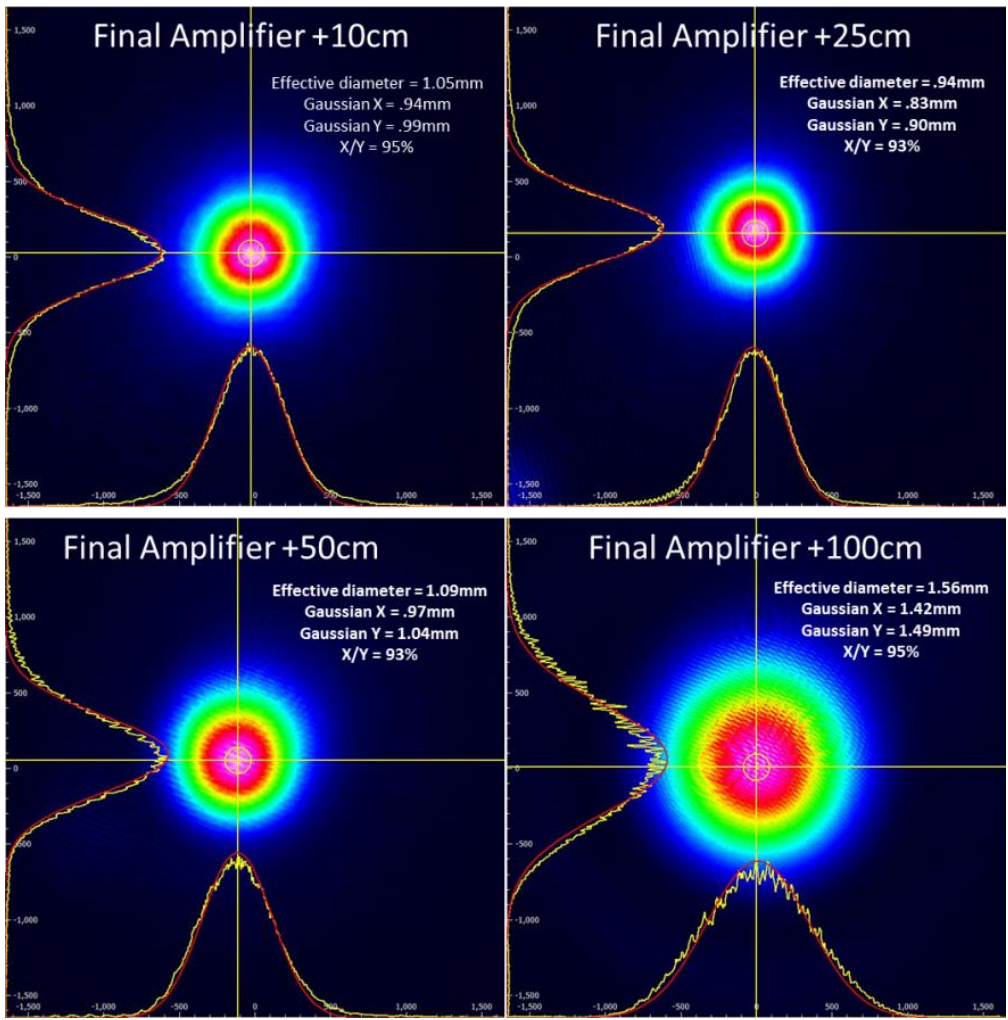

Figure 15: Beam size and shape as a function of propagation distance.

\section{Seed Laser Unit:}

The seed laser unit (SLU) supplies the transmitter with the seed wavelength for the DFG. The SLU contains six tunable diodes, a high speed switch, 99/1 fiber splitter, a fiber amplifier, and an isolator.

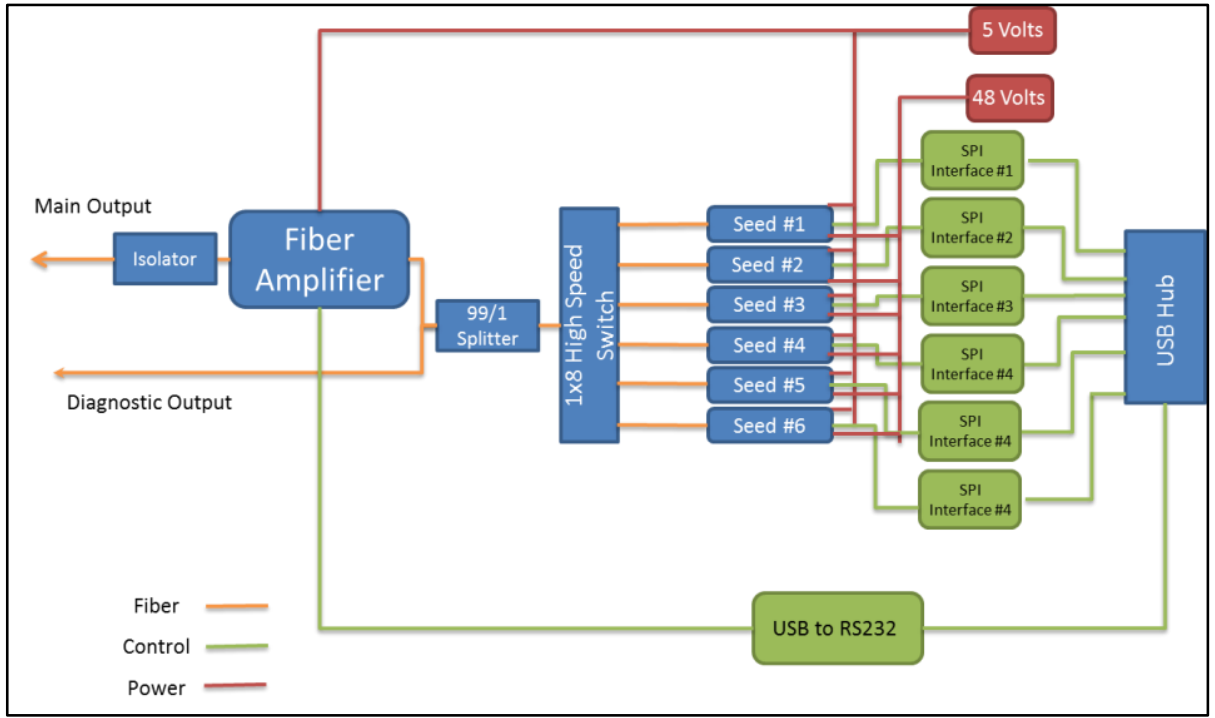

Figure 16: The block diagram of the seed laser unit. 


\section{Seed Lasers:}

The seed sources from Pirelli Broadband Solutions are mounted in an ILX Lightwave diode mount, shown in Figure 17. They are a continuous wave, dynamically tunable across the entire C-band (1530 to 1570 nanometers) in a 26 pin butterfly package. The external cavity mirror for these diodes is a liquid crystal device. The tuning is achieved by changing the voltage amplitude ( 2.5 - 45 Volts) on a square wave used to control the liquid crystal mirror. The original data from the manufacturer, Figure 18 , states that these diodes have a continuous and repeatable tuning curve as a function of voltage. $A 7^{\text {th }}$ order polynomial would be sufficient for the tuning requirements. As a result, LLNL designed and built our own controller boards that provide and monitor the operating current and temperature for each diode. These boards also provide the high resolution voltages to the square wave for the tuning mirror.
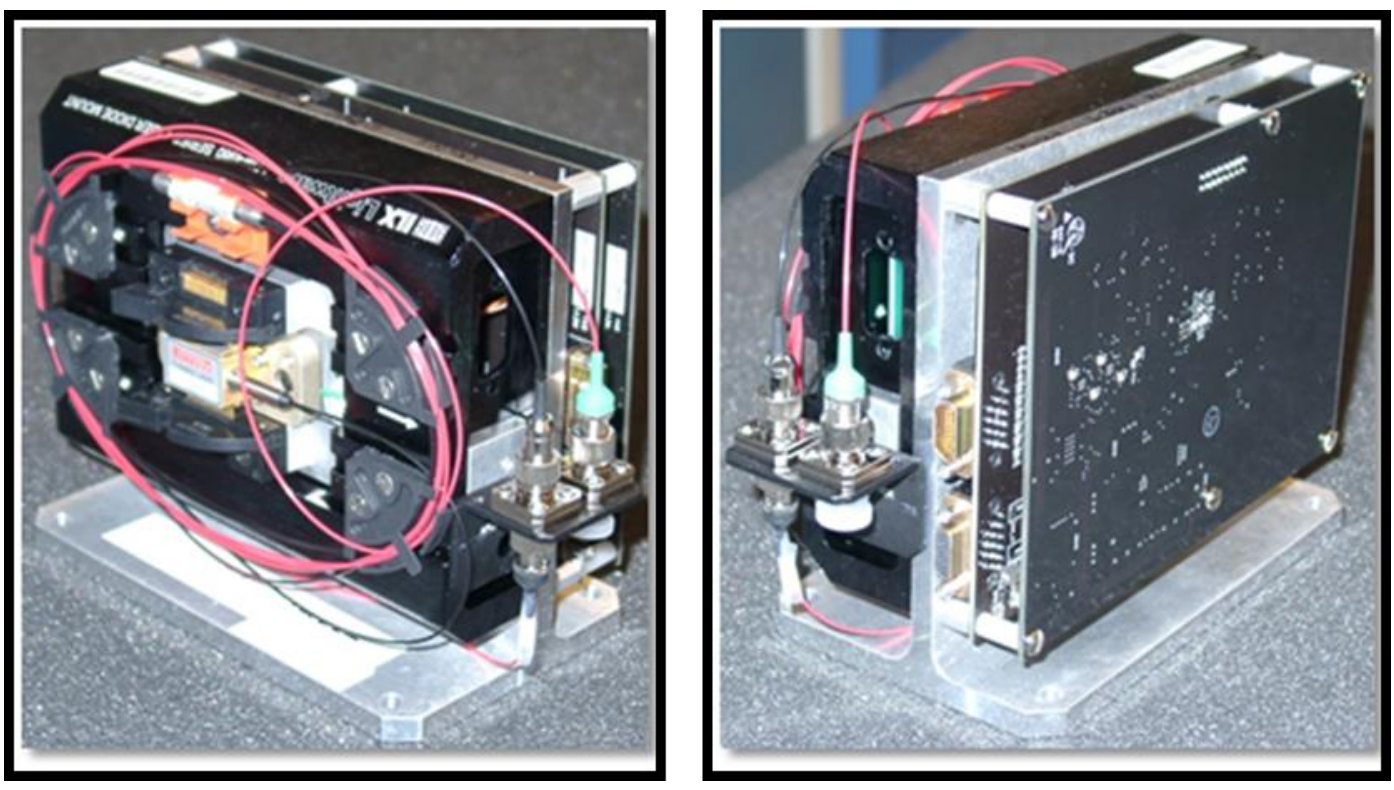

Figure 17: Picture of the Pirelli seed diode mounted with the LLNL designed control boards. 


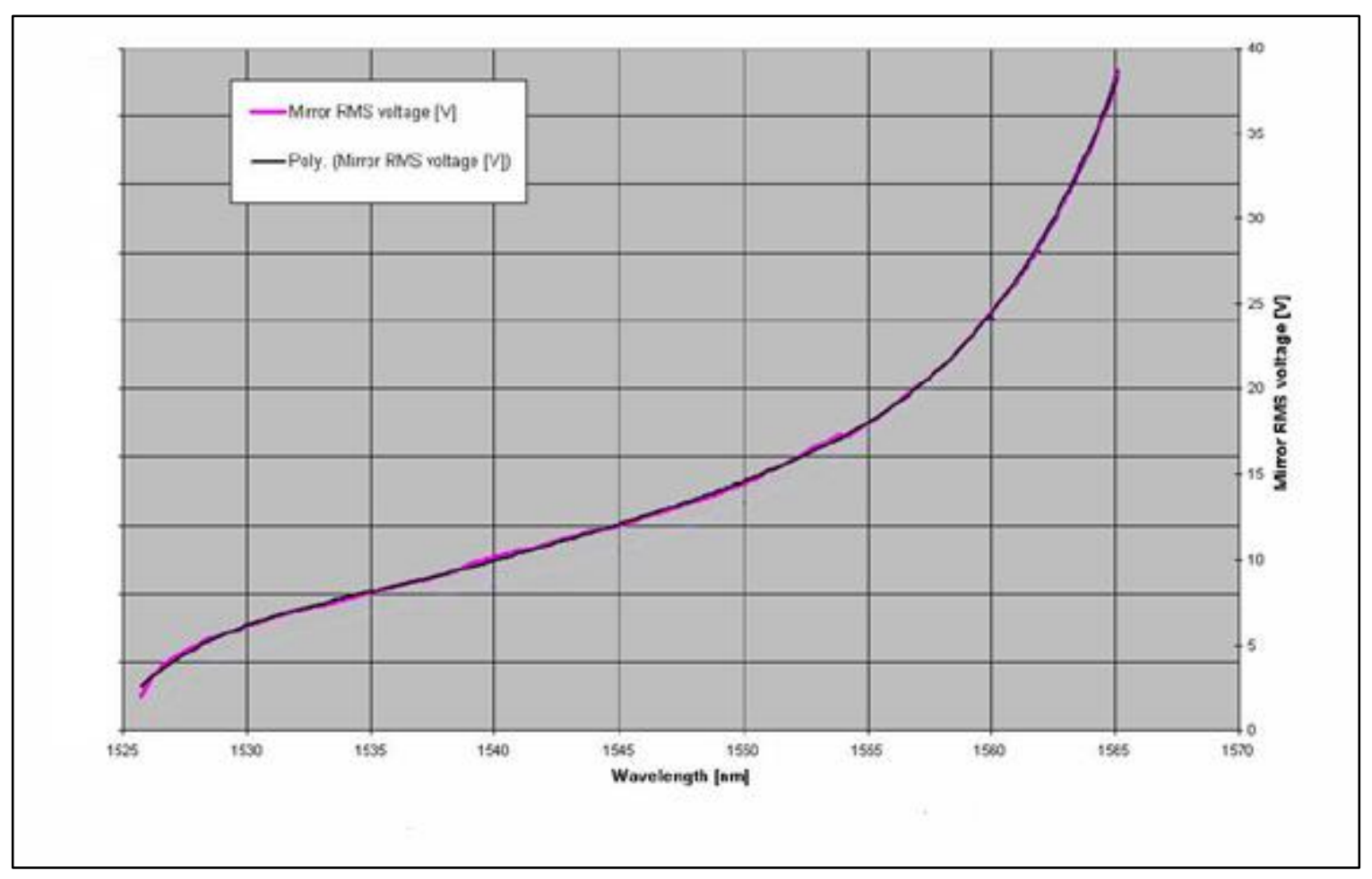

Figure 18: Diode tuning data from the manufacturer. (Little(4))

The control board provided a full tuning range of 16 bits, or 65,536 counts. The following graph is the result of a full tuning curve of one source from 500 counts to 65,634 in 2-step intervals at a gain current of $200 \mathrm{~mA}$ with a temperature of $22^{\circ} \mathrm{C}$ along with the $7^{\text {th }}$ order polynomial curve fit. Upon a closer inspection of the data, there is a clear series of discontinuities which appear to be caused by the laser mode-hopping. These discontinuities are approximately 65 picometers in wavelength or $8.25 \mathrm{GHz}$ in frequency. The original specification was to reach the wavelength to within $2 \mathrm{GHz}$, but because of these discontinuities it was clear that a curve-fit equation won't satisfy the requirements. As a result, another way must be found to tune the diodes to the wavelengths within the discontinuities. The solution $\left(\right.$ Little $\left.^{(4)}\right)$ to this issue is to tune not only the voltage of the tuning mirror, but also the gain current of the diode. 


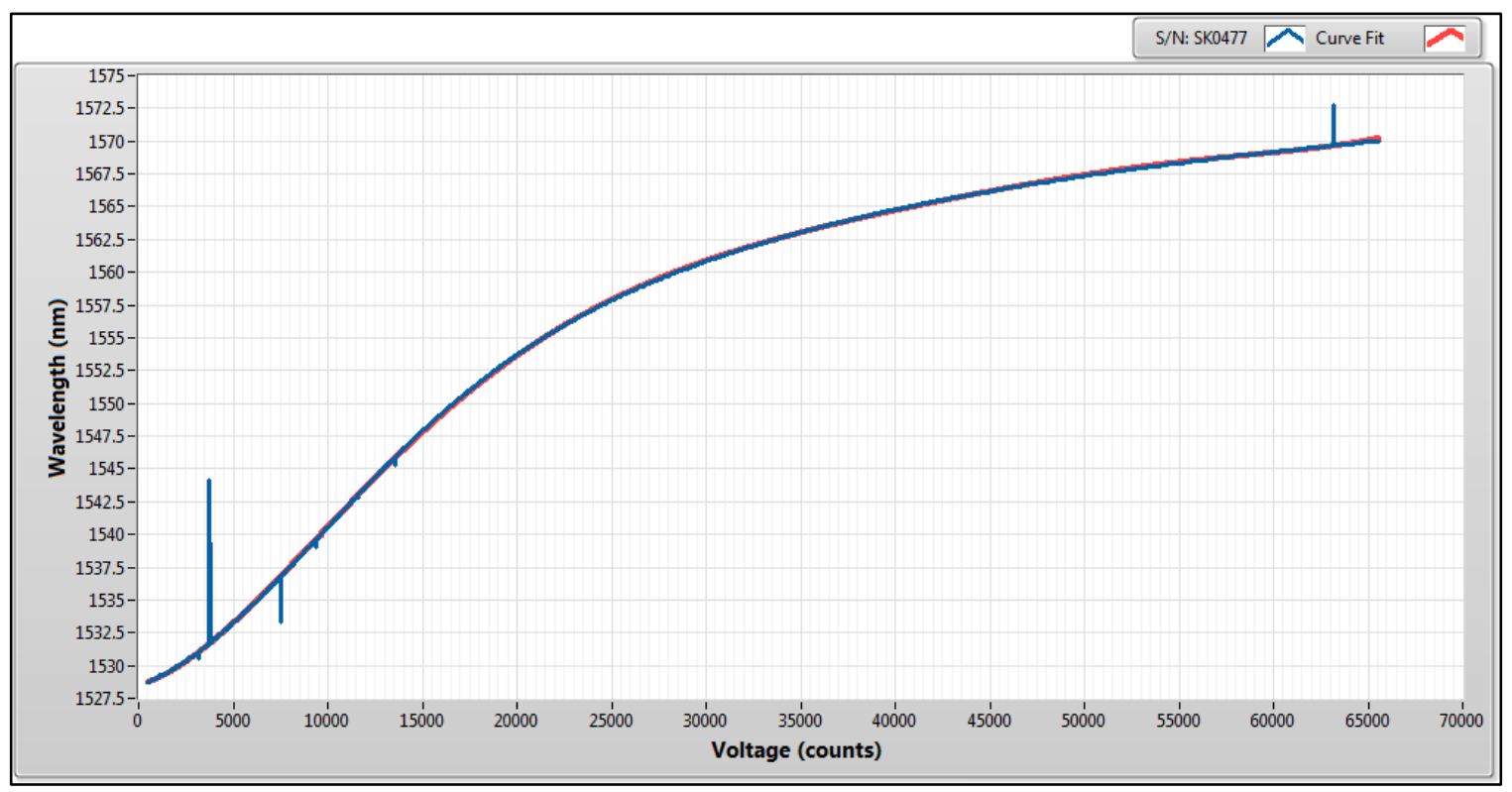

Figure 19: Measurement of the wavelength as a function of tuning mirror voltage.

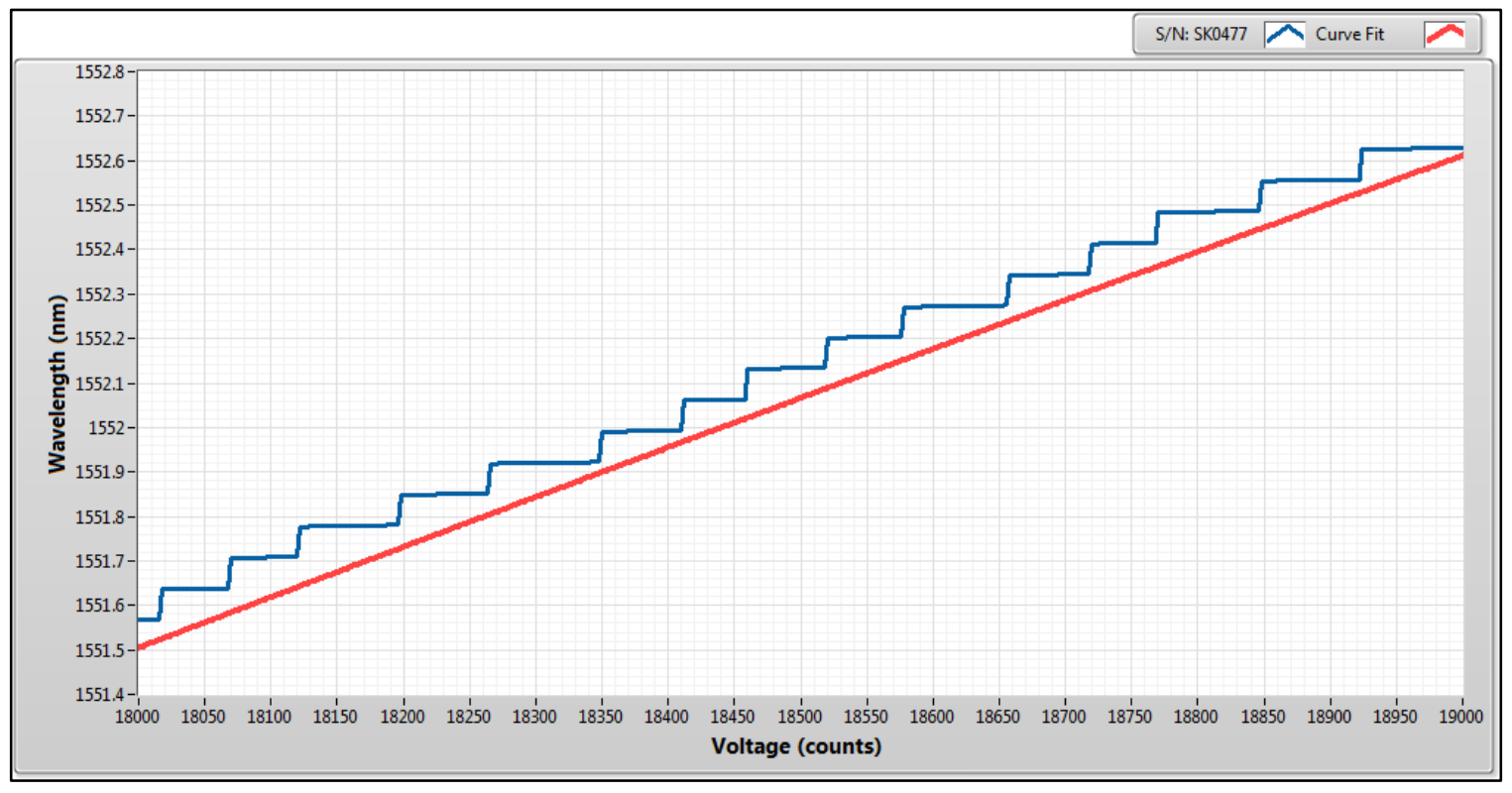

Figure 20: View of the discontinuities of the wavelength within the voltage scan.

It turns out that the increase in laser gain current causes the tuning curve to shift both upward and to the right as shown in the following graph. This means that the center of each step will increase with both the gain current and the tuning mirror voltage. 


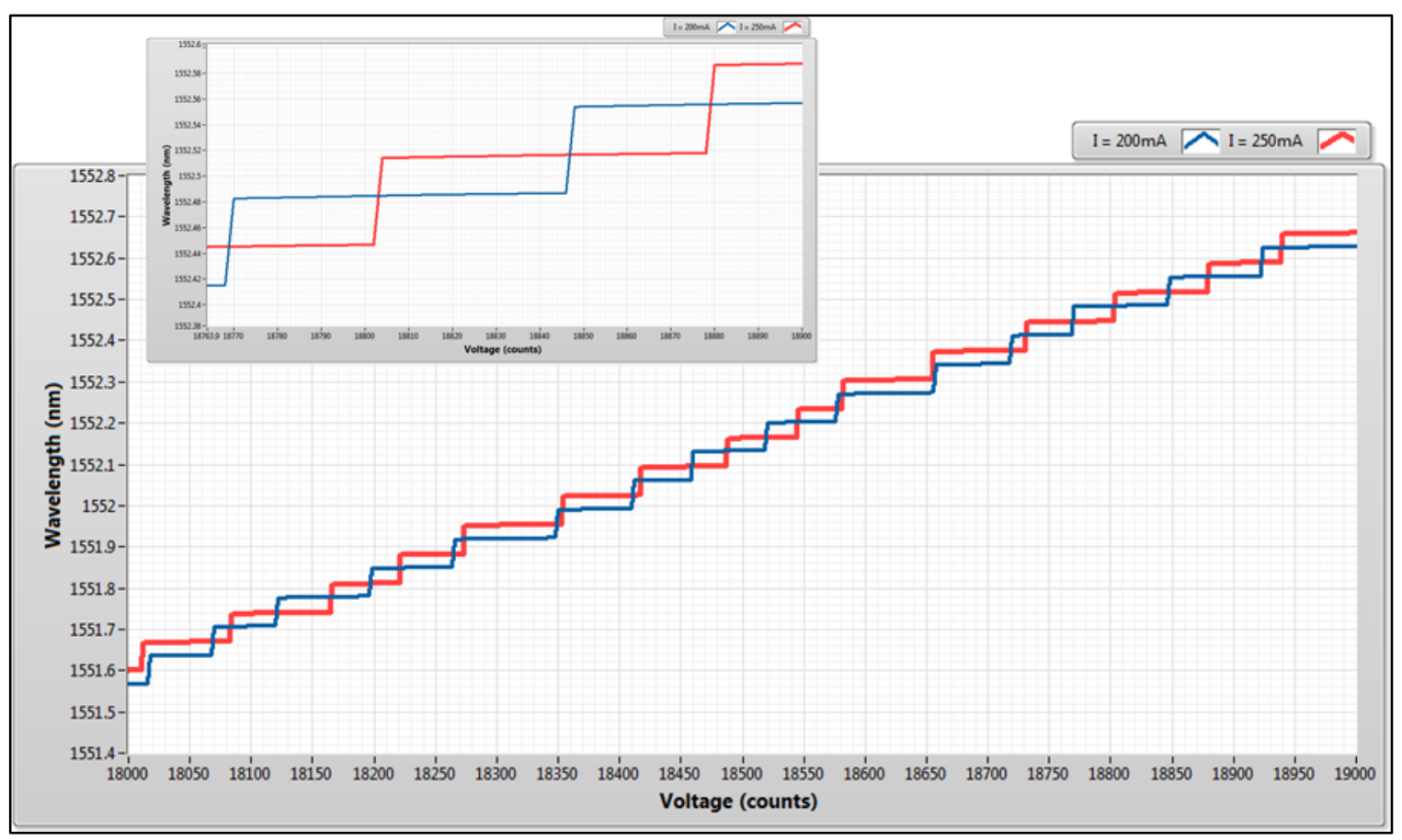

Figure 21: Shows the tuning curve of a diode at two gain current setting.

The difference of the wavelength of each step center as a function of the diode gain current is shown in Figure 22. Three different step centers are shown along with the simple curve fit in the form of $\lambda=$ $a I^{2}+b$, where $l$ is the gain current. The parameters $a$ and $b$ depend on the current and wavelength at the end of the collected data. Now that it is known how the wavelength changes as a function of gain current, the voltage change for the tuning mirror has to be determined. Since the steps are so small, the voltage variation is approximately linear and therefore can be determined by interpolation between adjacent voltages in a lookup table. 


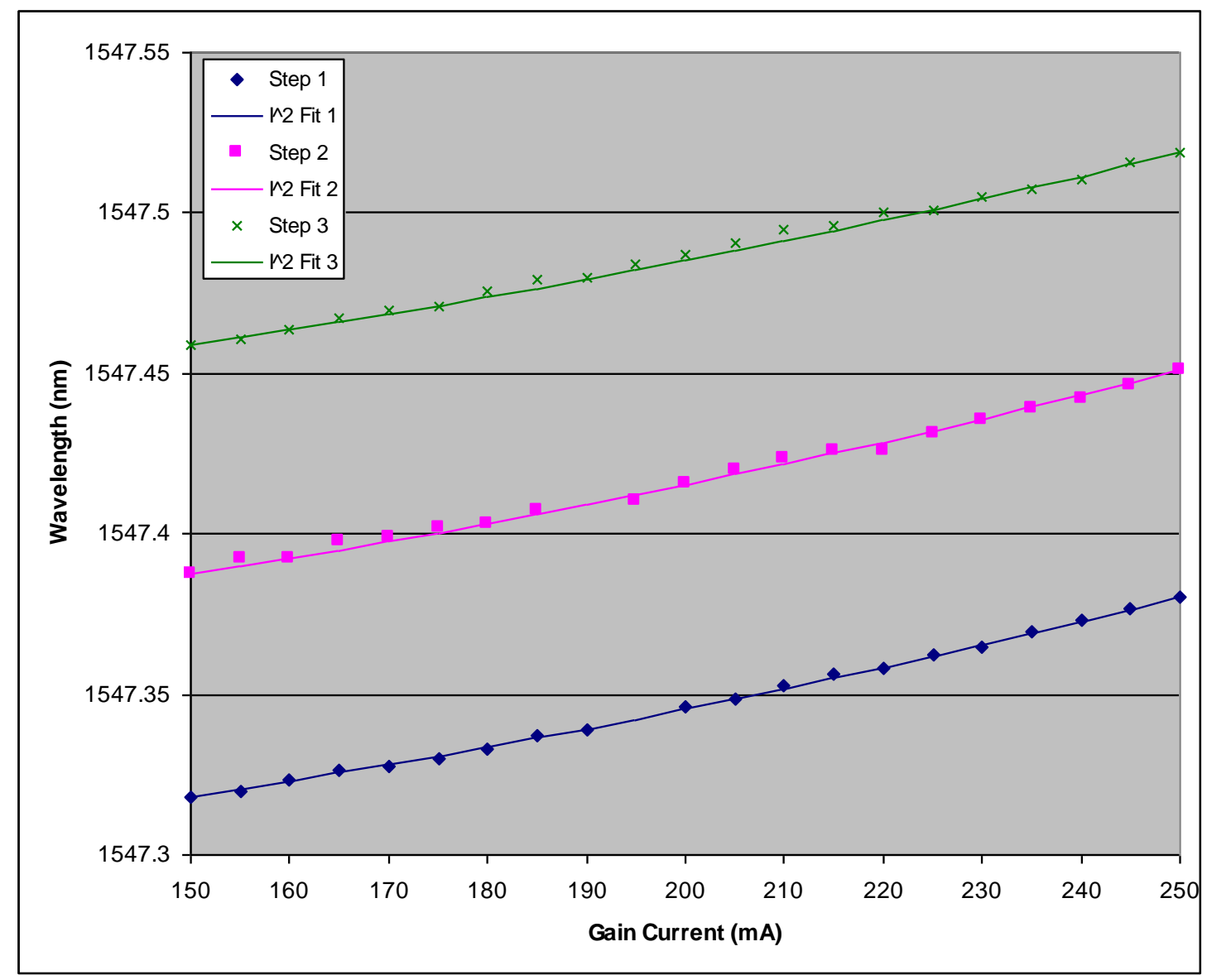

Figure 22: Variation of step center wavelength with current and curve fit with a simple relation to $I_{\text {gain }}^{2}\left(\operatorname{Little}^{(4)}\right)$

Before each of the seed diodes are installed into the system, they must first be characterized. To characterize the diodes, a full tuning scan $(500-65,534$ counts) at a given gain current $(200 \mathrm{~mA})$ is performed. Once completed, a tuning table was created which consists of two columns; one column is the voltage count at the center of each step and the second column is the wavelength at the corresponding step center. A second partial (15,000-30,000 counts) tuning scan was done at a gain current of $250 \mathrm{~mA}$. Again, the step centers were found and the average current slope was calculated over the step samples. Each diode has a configuration file that has the voltage counts and wavelength at each step center (tuning table), the gain current that the scan was performed at, and the calculated current slope.

Once a desired wavelength ( $\left.\lambda_{\text {desired }}\right)$ is identified, the software searches the tuning table to determine the closest wavelength and its associated tuning voltage and which two voltage steps that it lies between. Then, it calculates the new gain current by the following equation.

$$
I_{\text {new }}=\sqrt{\frac{\left(\lambda_{\text {desired }}-\lambda_{0}\right)}{a}+I_{0}^{2}}
$$


Where $\lambda_{0}$ is the closest wavelength in the tuning table, $I_{0}$ is the current at which the table was recorded, and $a$ is the current slope. In order to find the tuning voltage necessary to achieve the desired wavelength, uses the following equation.

$$
V_{\text {desired }}=\left(\frac{\lambda_{\text {desired }}-\lambda_{0}}{\lambda_{1}-\lambda_{0}}\right)\left(V_{1}-V_{0}\right)+V_{0}
$$

Where $V_{0}$ is the voltage at the closest step in the tuning table and $\lambda_{0}$ is it's corresponding wavelength; $V_{1}$ is the next closest voltage and $\lambda_{1}$ is it's corresponding wavelength; and $\lambda_{\text {desired }}$ is the desired wavelength.

In order to test the effectiveness of the algorithm, a LabVIEW program was written to scan the desired wavelength from $1535.000 \mathrm{~nm}$ to $1555.000 \mathrm{~nm}$ in increments of $0.005 \mathrm{~nm}$, which is approximately $0.62 \mathrm{GHz}$. This program followed the steps above by searching the diode configuration file and calculating the new gain current and voltage counts. The program would then change the parameters to the new calculated values and measure the wavelength using the Toptica Wavemeter. This process was repeated for every desired wavelength. The following graph shows the results of one of the tests for one diode.

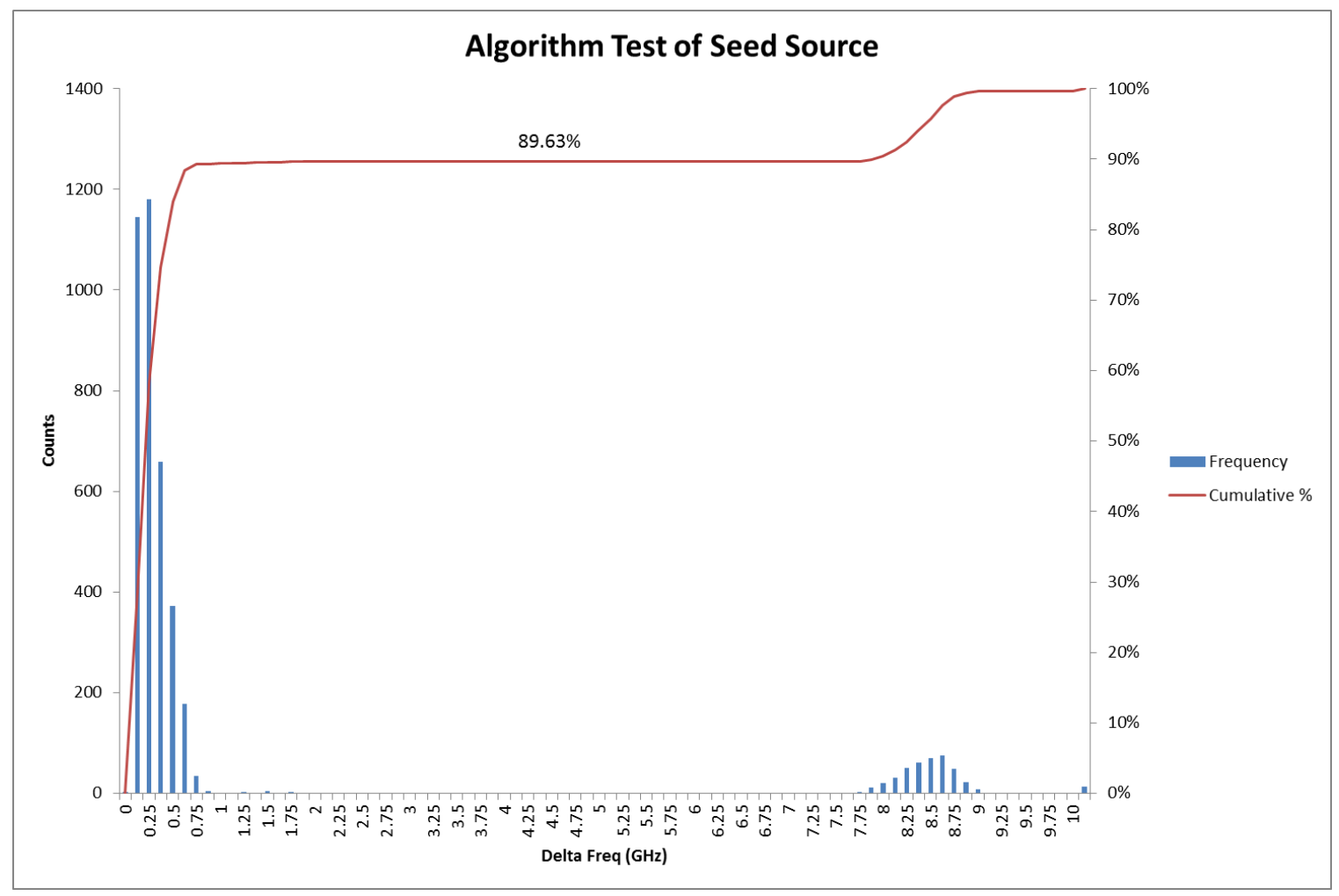

Figure 23: Shows the effectiveness of the algorithm for the seed sources.

As shown in the graph, approximately $90 \%$ of the measured wavelengths were within $2 \mathrm{GHz}$ of the desired wavelength. Another feature that came out from running this algorithm test was the location of the "bad zones" of each source. If time permitted, these "bad zone" locations could be added to each 
diode's configuration file. Therefore, the software could then choose which source to use for each desired wavelength.

\section{High Speed Switch:}

The purpose of the high speed fiber switch is to switch between the six diode seed sources within the period of the pump laser, which is 100 usec. The switch that was used was a Low-Insertion-Loss polarization maintaining $1 \times 8$ lithium niobate $\left(\mathrm{LiNbO}_{3}\right)$ Electro-Optical switch from EOSpace (shown below). To control the switch, three voltages are applied to $V_{1}, V_{2}$ and $V_{3}$, which were supplied by a National Instruments analog output module (NI 9264).

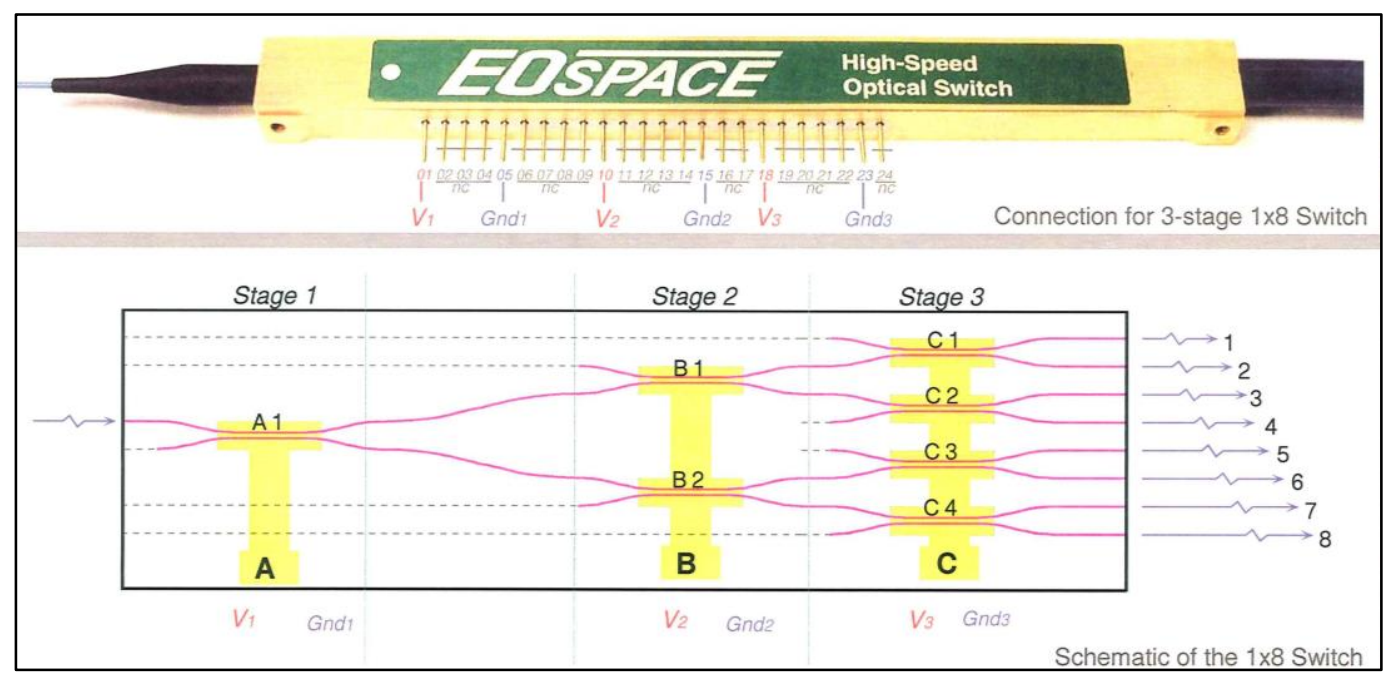

Figure 24: The picture and schematic of the EOSpace $1 \times 8$ switch.

To configure the voltages for each voltage channel, a diode source was coupled to the single side of the switch and two photodiodes were connected to the corresponding channels that were being tested. For example, if voltage channel $2\left(V_{2}\right)$ was being configured, meaning $V_{1}$ and $V_{3}$ were held constant, then the photodiodes were connected to fiber channels 1 and 4 . The voltage on $V_{2}$ was then adjusted until the photodiode on fiber channel 1 was maximized, this voltage is the $V_{2}$ cross voltage. The voltage on $V_{2}$ was then adjusted until the photodiode on fiber channel 4 was maximized; this voltage is called $V_{2}$ through voltage. The same procedure was done for $V_{1}$ and $V_{3}$, and the voltages were then saved in a switch voltage configuration file.

Now that the voltages for each channel have been configured correctly, the speed of the switch needed to be tested. To verify that the switching speed of the switch would meet the requirements, a diode source again was connected to the single side of the switch and two photodiodes connected to the two corresponding fiber channels. A LabVIEW program was written that would switch the voltages to the channels when a trigger signal was detected. Again, the voltages were supplied by the National Instruments analog output module and the trigger input was monitored by a National Instruments high speed digital I/O module (NI 9402). 


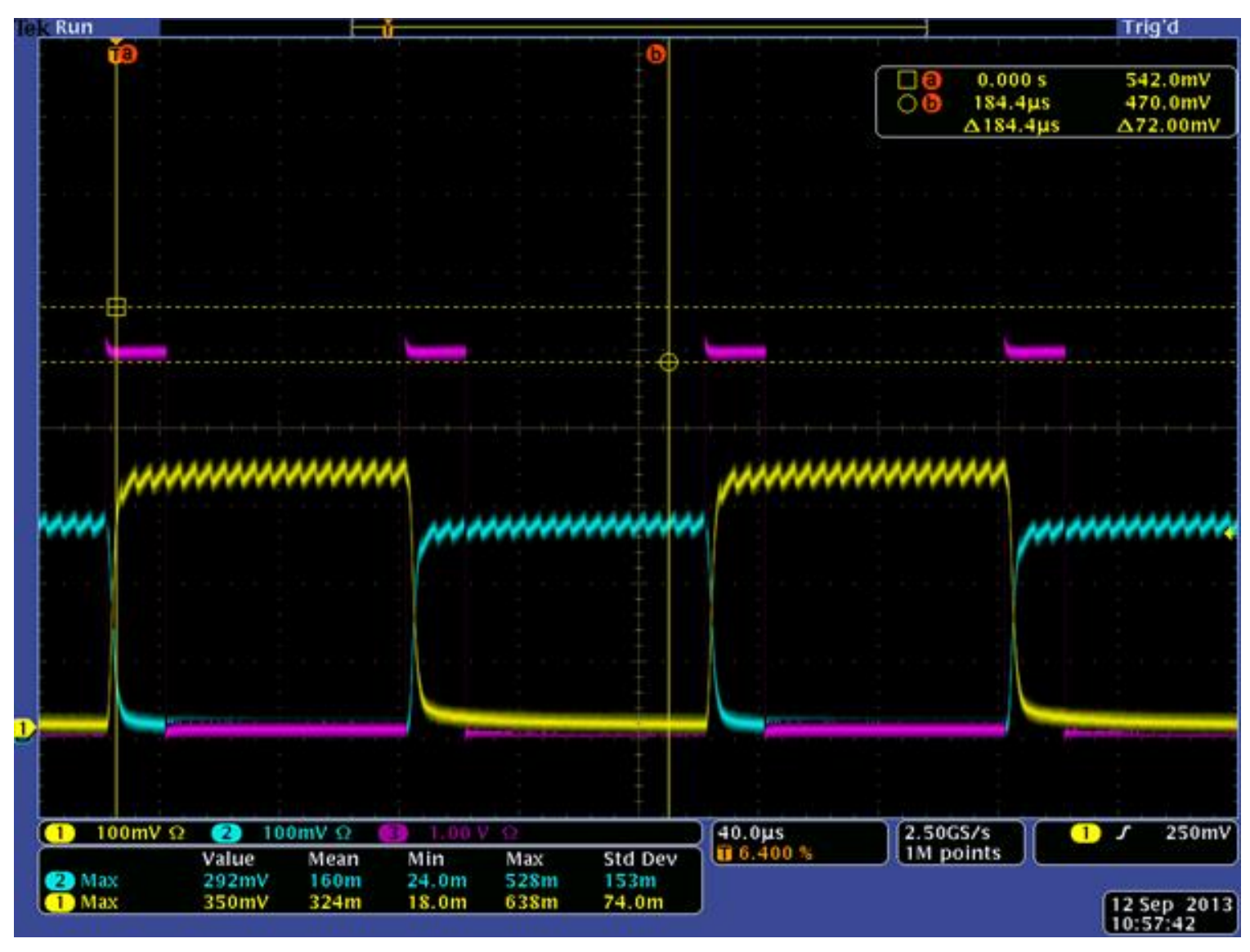

Figure 25: Measurement of the switching speed of the EOSpace high speed switch.

In the figure above, channel 3 (pink) is the trigger input running at $10 \mathrm{KHz}$. Channels 1 (yellow) and 2 (blue) are the voltages from the photodetectors on two fiber channels of the high speed switch. It takes approximately 16 microseconds for one channel to switch off and the other to switch on, which means it is plenty fast enough for this application.

\section{Fiber Amplifier:}

The final major component in the Seed Laser Unit is an erbium doped fiber amplifier (EDFA). The EDFA is a commercially produced unit from IPG Photonics. The EAM module was chosen because of its compact and ready-to-use form. It is driven by an external DC power supply, and the operational parameters can be externally set through an RS232 serial port. This amplifier is a continuous wave polarization-maintaining amplifier that boosts the power from the seeds from approximately 20 milliwatts to an output up to 2 watts with a spectral range from 1532 to 1567 nanometers.

\section{Pointing \& Centering Loop:}

The pointing \& centering loop is an automatic alignment system that is designed to maintain the pump laser's alignment through the transmitter. The system consists of two motorized mirror mounts with tip/tilt control and two position-sensing detectors; the basic design is shown in the diagram below. The original transmitter used two quad cells to determine the position of the laser. One of the upgrades done to the transmitter was to replace the two quad cells with two $1280 \times 1024$ USB CCD cameras from 
ThorLabs. This upgrade had two advantages; first the cameras provided a higher resolution image when calculating the position of the laser, and second, they provided a way to monitor the spatial profile of the pump beam.

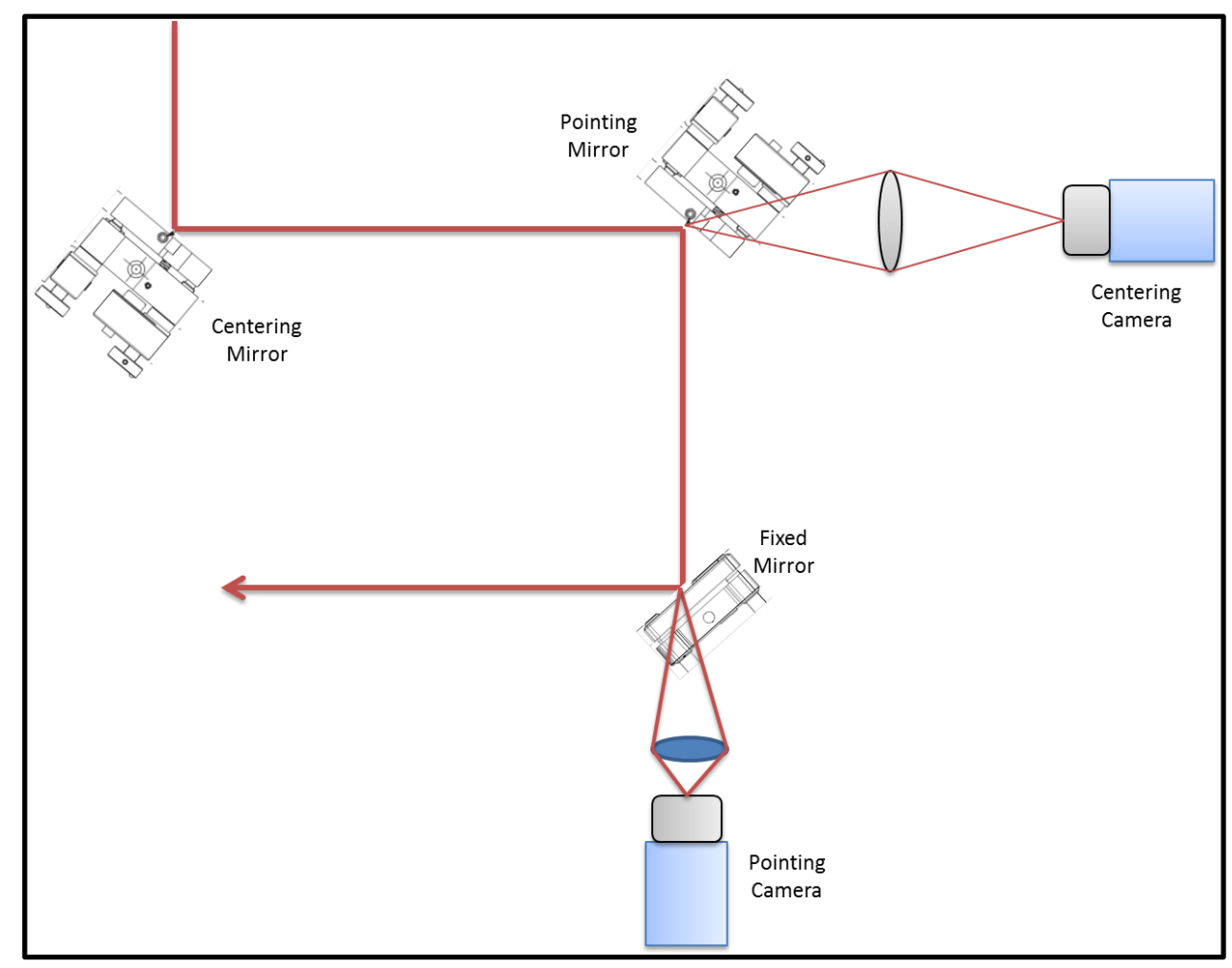

Figure 26: The basic diagram of the pointing and centering loop.

The basic concept of the pointing \& centering loop is this; the centering camera is used to monitor the near field while the pointing camera monitors the far field. Using the leak through of the pointing mirror, the face of the pointing mirror is imaged onto the centering camera. For the pointing mirror, the leak through the fixed mirror is focused onto the pointing camera. Once the cameras are aligned, the control system calculates the centroid of each image to determine the beam position. If the beam position is off from the setpoints, the control system adjusts the pointing and centering mirrors to align the centroids.

\section{Optical Parametric Amplification:}

(Note: This was not intended to be a nonlinear optics study; therefore the equations here were used to select the parameters of the crystals.)

Optical Parametric Amplification is based on the lowest-order nonlinear susceptibility $\chi^{(2)}$ of a nonlinear crystal material. The susceptibility is responsible for the three-wave mixing processes that are used to generate the wavelength necessary for this application. These processes are known as difference frequency generation (DFG) and optical parametric amplification (OPA). These processes use an 
interaction between three different wavelengths which have angular frequencies of $\omega_{1}, \omega_{2}, \omega_{3}$ (the indices indicate the order of the frequency). The pump frequency is $\omega_{3}$, the signal frequency is $\omega_{2}$, and $\omega_{1}$ is called the idler frequency, which is generated by the interaction of the other two frequencies.

The three wave-mixing process can be described by the interaction of the frequencies in the nonlinear crystal by a set of nonlinear coupled differential equations. The three coupled wave equations described by Träger ${ }^{(9)}$ are:

$$
\begin{aligned}
& \frac{\partial E_{1}(z)}{\partial z}=i \kappa_{1} E_{3}(z) E_{2}^{*}(z) e^{i \Delta k z} \\
& \frac{\partial E_{2}(z)}{\partial z}=i \kappa_{2} E_{3}(z) E_{1}^{*}(z) e^{i \Delta k z} \\
& \frac{\partial E_{3}(z)}{\partial z}=i \kappa_{3} E_{1}(z) E_{2}(z) e^{-i \Delta k z}
\end{aligned}
$$

with the coefficients $\kappa_{i}=\omega_{i} d_{e f f} / n_{i} c_{0}(i=1,2,3)$, $\mathrm{d}_{\mathrm{eff}}$ is the nonlinear coefficient, $\mathrm{z}$ is the propagation direction and the phase mismatch

$$
\Delta k=k_{3}-k_{2}-k_{1}
$$

Depending on the initial conditions of the three waves, these three equations can describe the various types of three wave-mixing processes generated by the single pass propagation of the light through the crystal.

\section{Difference Frequency Generator (DFG):}

Difference frequency generation is a nonlinear process that involves combining two photons of different energies to produce a third photon whose energy equals the difference between the two incident photons. In this case, the light from the MOPA is mixed with the light from the SLU in a periodically poled lithium niobate (PPLN) crystal. The output wavelength, known as the idler, is related to the two incident wavelengths by a conservation of energy equation.

$$
\begin{gathered}
\hbar \omega_{p}-\hbar \omega_{s}=\hbar \omega_{i} \rightarrow \omega_{i}=\omega_{p}-\omega_{s} \\
\text { or } \\
\frac{1}{\lambda_{i}}=\frac{1}{\lambda_{p}}-\frac{1}{\lambda_{s}}
\end{gathered}
$$

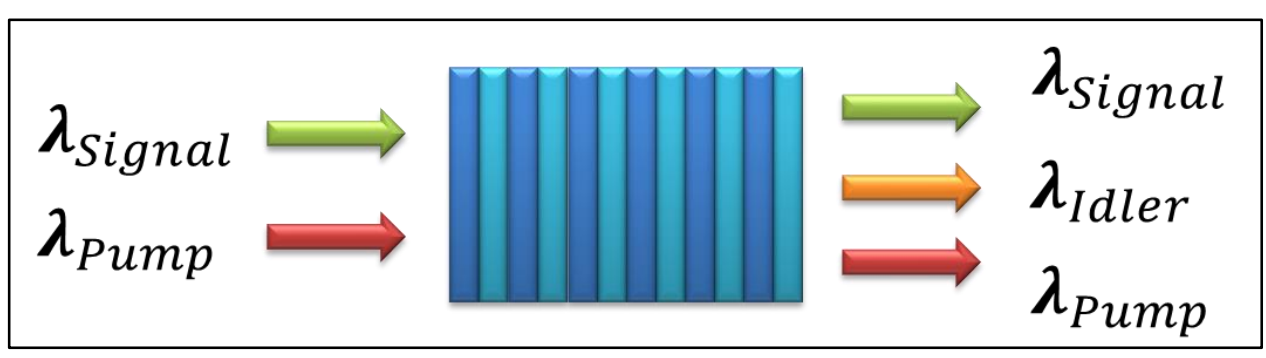

Figure 27: Diagram of the difference frequency generation technique used. 
Where $\lambda_{p}$ and $\lambda_{s}$ are the pump wavelength and the signal wavelength respectively. The highest conversion occurs at the center of the phase matching peak. This peak occurs when the conservation of momentum condition is met.

$$
\Delta k=k_{p}-k_{s}-k_{i}=0
$$

Where $\Delta k$ is the phase mismatch, and $k_{p}, k_{s}$, and $k_{i}$ are the wavevectors of the pump, signal, and idler beams, respectively. Since the wave propagation is collinear, the wavevectors can be replaced by $|k|=$ $n c / \lambda$, and therefore changed to:

$$
\frac{1}{\Lambda}=\frac{n_{p}}{\lambda_{p}}-\frac{n_{s}}{\lambda_{s}}-\frac{n_{i}}{\lambda_{i}}
$$

Where $n_{p}, n_{s}$, and $n_{i}$ are the extraordinary refractive indexes of PPLN at the pump, signal and idler wavelengths. The poling period, also known as the grating period, is given by $\Lambda$. The index of refraction is calculated using Sellmeier equation that is described by Jundt ${ }^{(6)}$.

$$
n_{e}{ }^{2}=a_{1}+b_{1} f+\frac{a_{2}+b_{2} f}{\lambda^{2}-\left(a_{3}+b_{3} f\right)^{2}}+\frac{a_{4}+b_{4} f}{\lambda^{2}-a_{5}{ }^{2}}-a_{6} \lambda^{2}
$$

The temperature parameter $f$ is the square of the absolute temperature in degrees Kelvin, with an added offset to make it vanish at the reference temperature of $T_{o}$. For temperatures $T$ expressed in degrees Celsius, $f$ is given by the follow equation ${ }^{(6)}$ :

$$
\begin{gathered}
f=\left(T-T_{o}\right)\left(T+T_{o}+2 * 273.16\right) \\
f=\left(T-24.5^{\circ} C\right)(T+570.82)
\end{gathered}
$$

The parameters for calculating the index of refraction using Sellmeier equation are giving in the following table.

\begin{tabular}{|c|c|}
\hline Parameter & Value \\
\hline $\mathrm{a}_{1}$ & 5.35583 \\
\hline $\mathrm{a}_{2}$ & 0.100473 \\
\hline $\mathrm{a}_{3}$ & 0.20692 \\
\hline $\mathrm{a}_{4}$ & 100 \\
\hline $\mathrm{a}_{5}$ & 11.34927 \\
\hline $\mathrm{a}_{6}$ & $1.5334 \times 10^{-2}$ \\
\hline $\mathrm{b}_{1}$ & $4.629 \times 10^{-7}$ \\
\hline $\mathrm{b}_{2}$ & $3.862 \times 10^{-8}$ \\
\hline $\mathrm{b}_{3}$ & $-0.89 \times 10^{-8}$ \\
\hline $\mathrm{b}_{4}$ & $2.657 \times 10^{-5}$ \\
\hline
\end{tabular}

Table 1: Fitted Parameters for Sellmeier equation, Jundt(6).

When choosing the poling period and the temperature of the crystal, the thermal expansion of the crystal must be taken under consideration. Under elevated temperatures, the crystal expands along the propagation direction and therefore increases the poling period. The thermal expansion coefficients ${ }^{(6)}$ 
$\alpha$ and $\beta$ describe the PPLN crystal length at temperature $T$ normalized to the length of the crystal at $25^{\circ} \mathrm{C}$

$$
l=l_{25^{0} C}\left[1+\alpha\left(T-25^{0} C\right)+\beta\left(T-25^{0} C\right)^{2}\right]
$$

where $\alpha=1.54 \times 10^{-5} K^{-1}$ and $\beta=5.3 \times 10^{-9} K^{-2}$.

Using the above equations with a pump wavelength of 1064.3848 nanometers, the signal wavelength range from 1.53 micron to 1.57 microns, and a crystal temperature of $180^{\circ} \mathrm{C}$, the following figure shows how the poling period changes in order to satisfy the phase-matching criteria. The vertical bars at 3.3 microns and 3.5 microns represent the range of the idler wavelength produced with the given signal wavelengths.

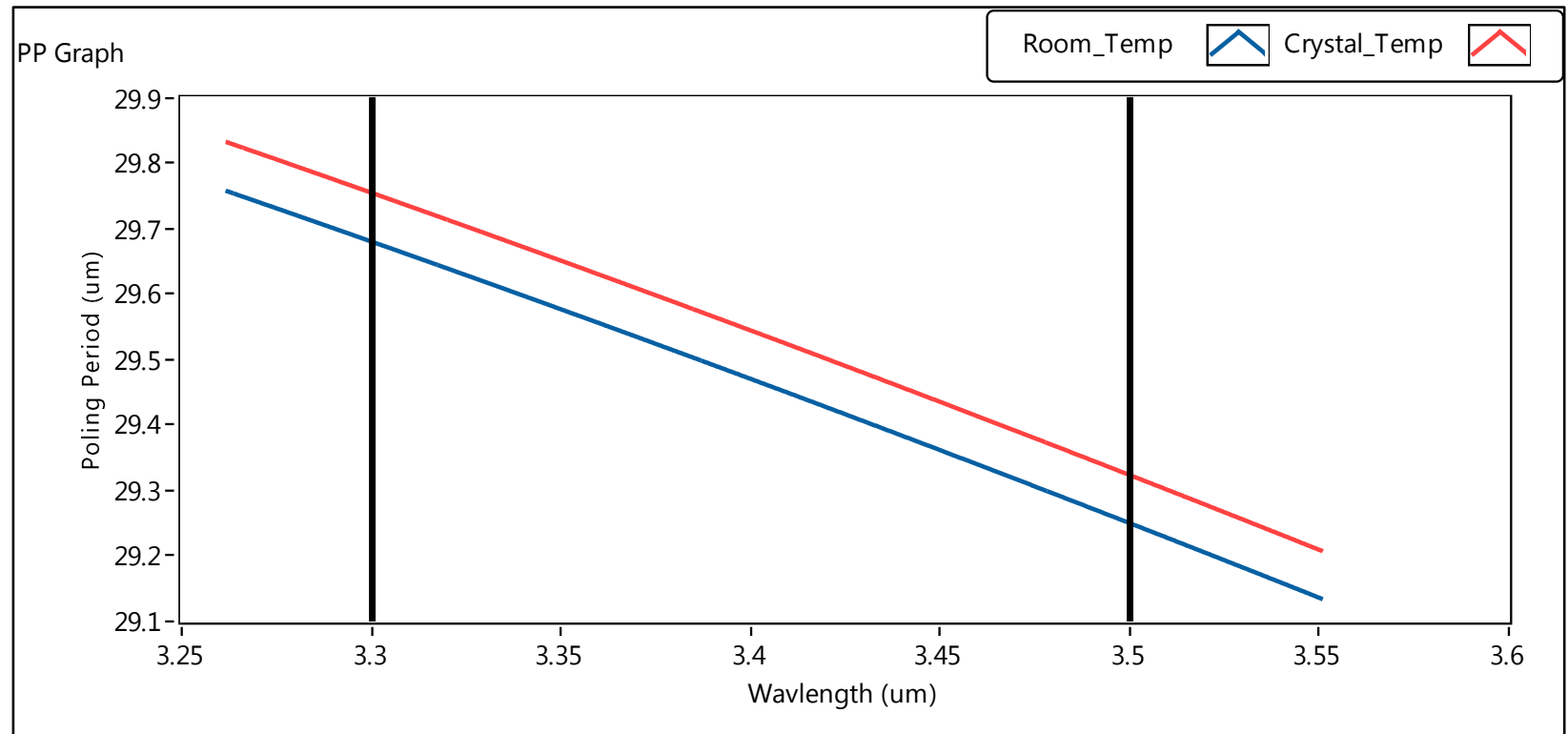

Figure 28: Graph showing how the poling period changes as a function of the idler wavelength with the crystal at room temperature and at $180^{\circ} \mathrm{C}$.

Now that the poling period has been calculated, we need to look at the input angles of the pump and signal beams to make sure they are reasonable. The figure above shows that a poling period of 29.3um will meet the phase matching criteria for the full wavelength range. To achieve an output wavelength of 3.5 micron the angle of the pump and signal is near zero. To achieve an output of 3.3 microns the poling period is approximately $29.7 \mathrm{um}$. The angle to achieve this effective poling period is given by $\cos \theta=$ $\Lambda / \Lambda_{\text {eff }}$ and using Snell's law to determine the external angle. As shown in the following figure, the external angle necessary to achieve phase-matching over the full range is a little excessive. 


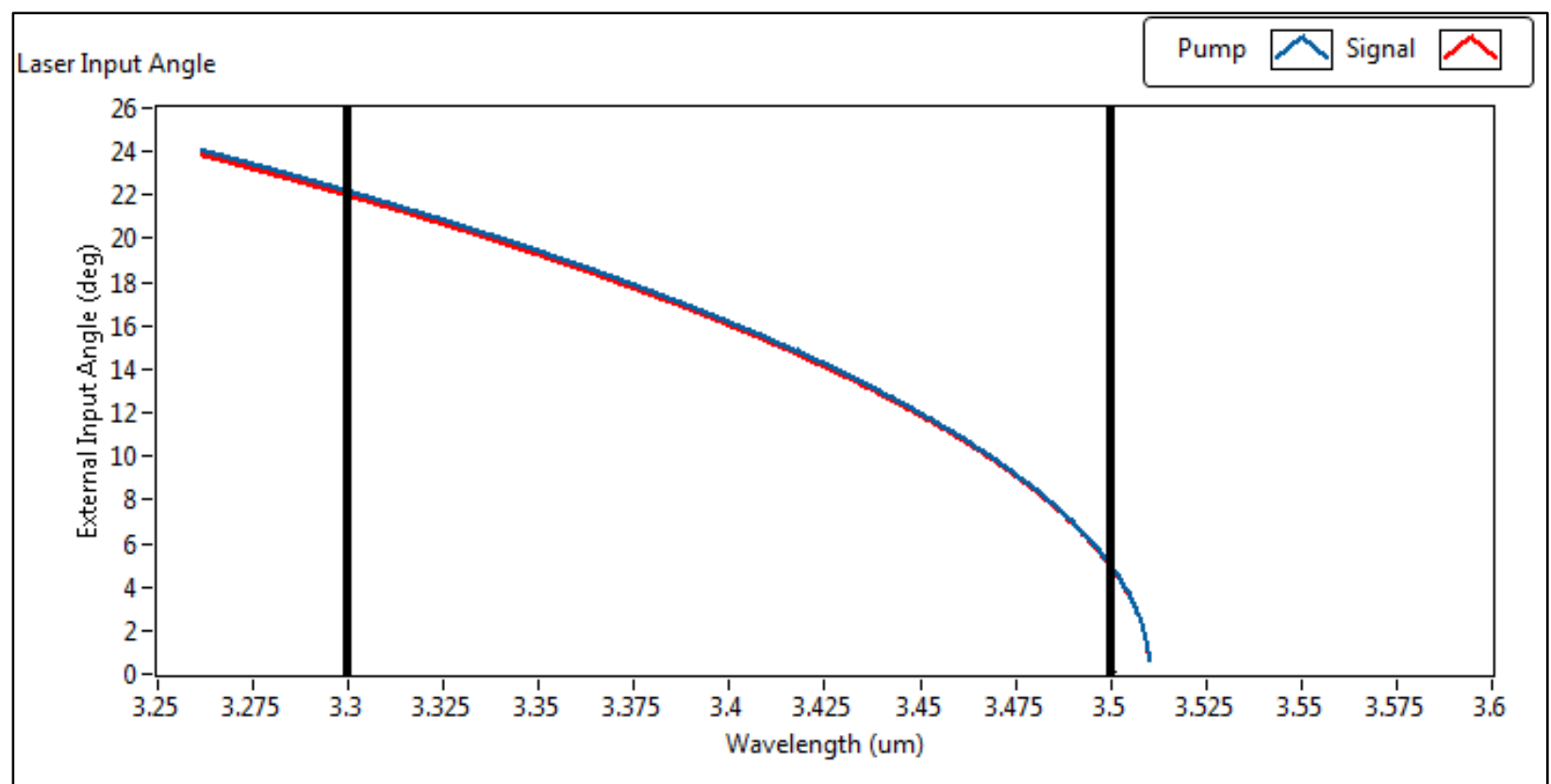

Figure 29: The external input angle of the pump and signal beam to phase-match with a poling period of 29.3 microns.

To reduce the external input angle, the angle of the poling period was increased along with reducing the poling period. After some careful consideration, a poling angle of $35^{\circ}$ with a poling period of 23.9 micron was chosen.

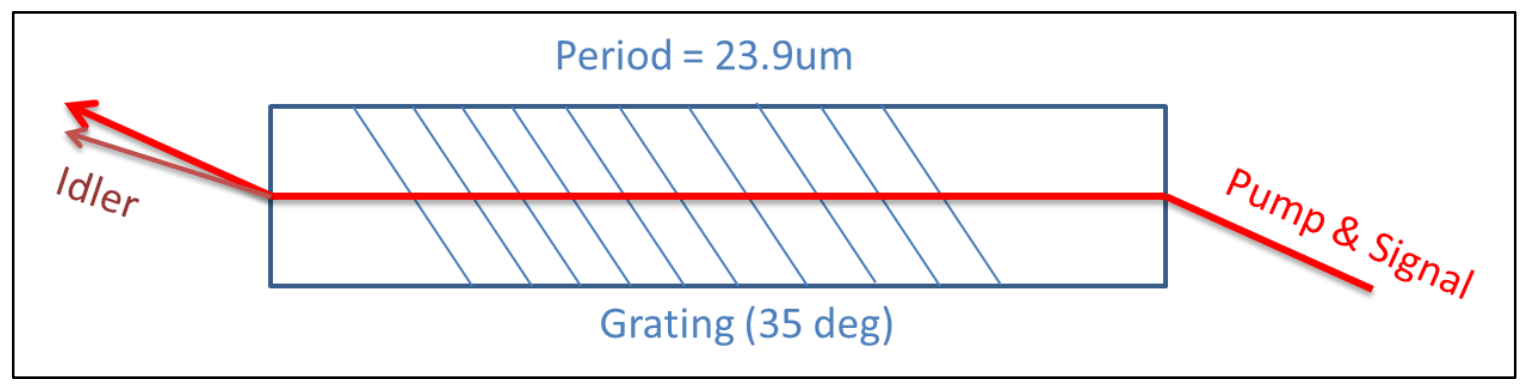

Figure 30: Sketch of the PPLN crystal used in the DFG. 


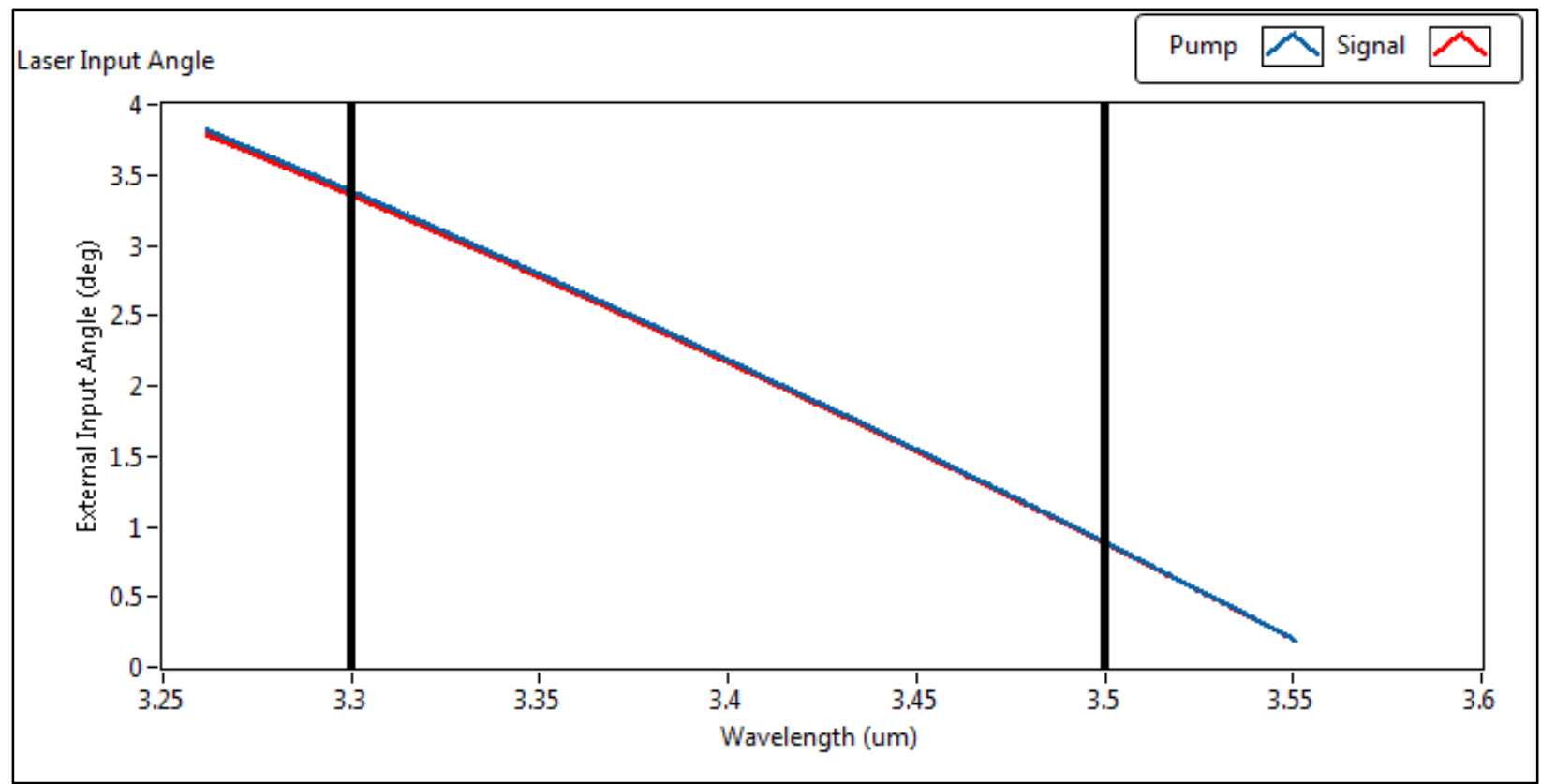

Figure 31: The external input angle of the pump and signal beam to phase-match with a poling period of 23.9 microns at an angle of $35^{\circ}$.

The last specification that needs to be determined is the length of the crystal. This can get very complicated due to the laser parameters, such as intensity, beam profile, and pulse shape, as they propagate through the crystal. Then, there are the crystal parameters, such as the group velocities, group delay dispersion, effective nonlinearity, and its coupling and threshold power that must be taken into account to accurately model the performance of the PPLN crystal. To the first order, the single pass gain of the PPLN crystal determines the length needed. Neglecting any pump depletion $\left(d E_{p} / d z=0\right)$ and assuming that only the pump and signal beams (meaning $E_{i}=0$ ) are present at the crystal face $(z=0)$, then the coupled wave equations can be solved analytically (Träger $\left.{ }^{(9)}\right)$. The single pass fractional gain is obtained by

$$
G_{i}(l)=\Gamma^{2} l^{2} \frac{\sinh ^{2}(g l)}{(g l)^{2}}
$$

where $/$ is the length of the nonlinear material and $g$ is the total gain factor.

$$
g=\sqrt{\Gamma^{2}-\left(\frac{\Delta k}{2}\right)^{2}}
$$

The parametric gain factor $\Gamma$ is

$$
\Gamma^{2}=\frac{2 \omega_{s} \omega_{i} d_{e f f}{ }^{2} I_{p}}{n_{p} n_{s} n_{i} \varepsilon_{0} c^{3}}
$$

where $d_{\text {eff }}$ is the effective nonlinear coefficient of PPLN and $I_{p}$ is the intensity of the pump beam. 
The design goal for the DFG was 1 watt of output power with 45-50 watts of pump power going to the OPA. To achieve this, the pump was split with roughly $20-25 \%$ of the power going to the DFG. The following graph shows the power of the pump to both the DFG and OPA as a function of the power control waveplate.

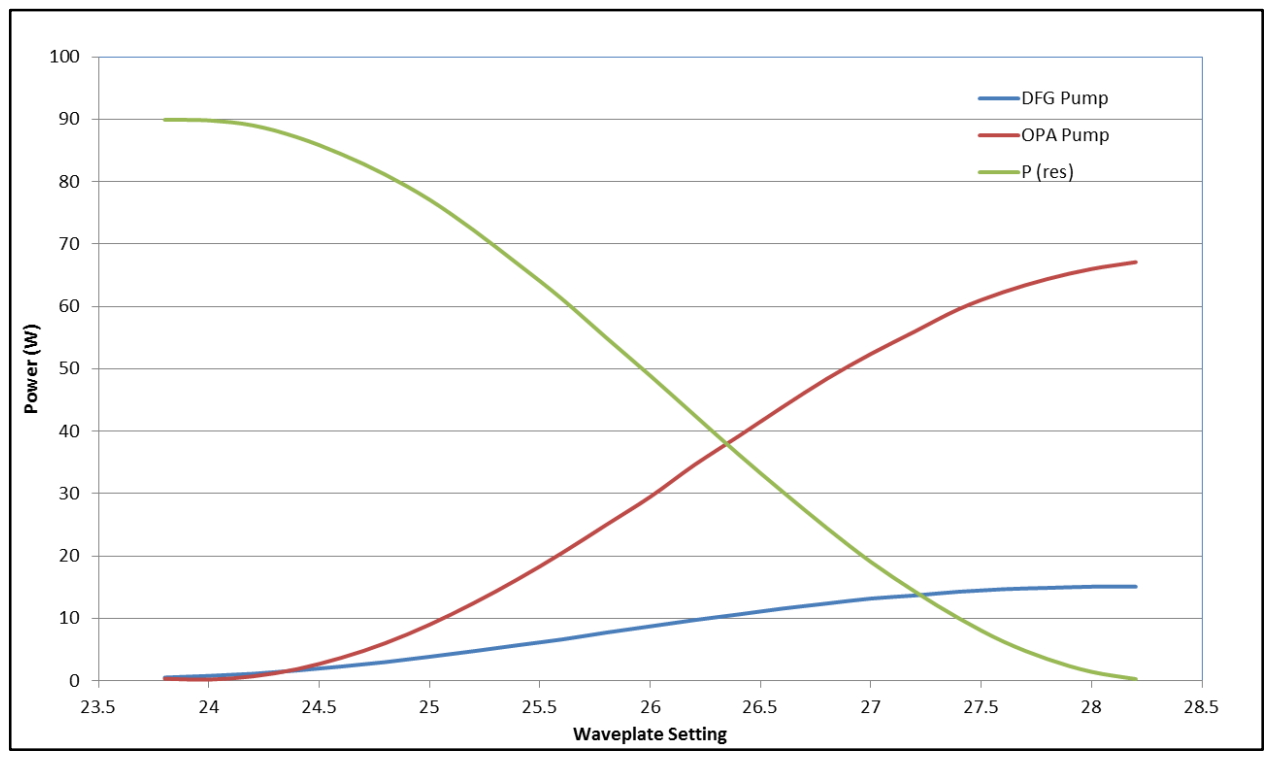

Figure 32: Shows the split ratio of the pump power to the DFG and OPA.

With 15 watts of pump power going to the DFG crystal, a pulse-width of 20 nanoseconds, and a beam diameter of roughly 600 microns (shown below), this results in a pump intensity of around $265 \mathrm{GW} / \mathrm{m}^{2}$.

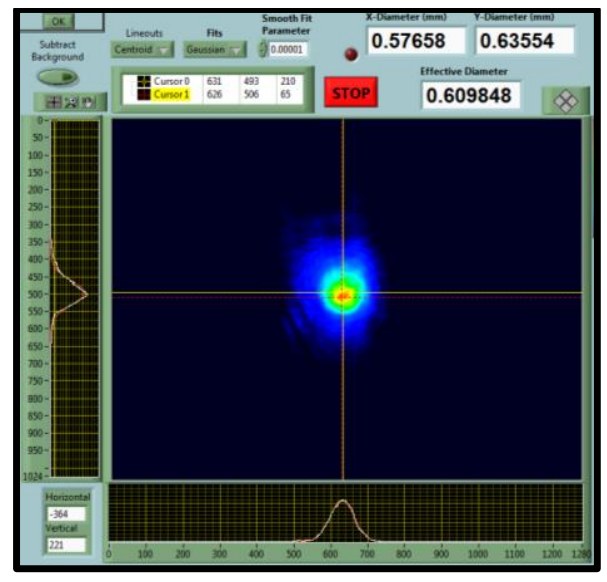

Figure 33: The pump size at the DFG crystal.

Inserting the pump intensity into the parametric gain factor equation from above, along with a $d_{\text {eff }}$ of $14.8 \mathrm{pm} / \mathrm{V}\left(\mathrm{Smith}^{(8)}\right)$ for the PPLN crystal, the single pass gain for the DFG is shown in the graph below. The different plots represent the gain at the ends of the required wavelength range. 


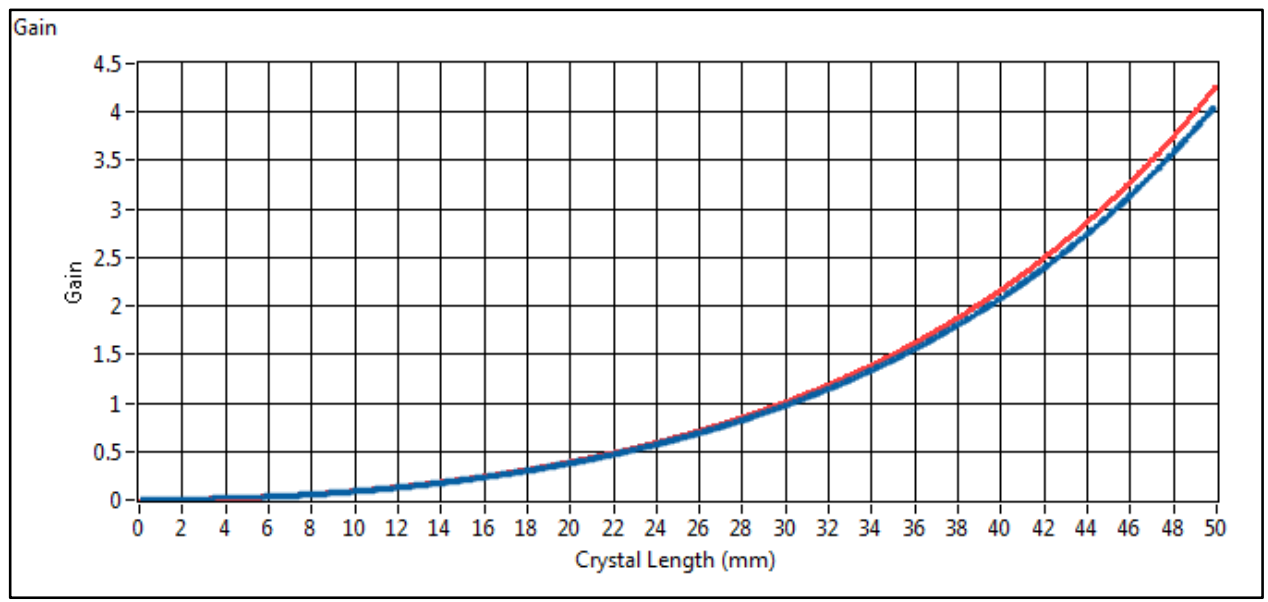

Figure 34: The plot of the single pass gain of the DFG as a function of crystal length.

In reality, the crystal length was chosen empirically. Multiple crystals were purchased with a poling period of 23.9 micron at an angle of $35^{\circ}$ in three different lengths; $30 \mathrm{~mm}, 40 \mathrm{~mm}$, and $50 \mathrm{~mm}$. The dimensions of the PPLN crystals are $1 \mathrm{~mm}$ thick by $10 \mathrm{~mm}$ wide by $30 / 40 / 50 \mathrm{~mm}$ long. The goal was to get out the highest gain possible without damaging the crystal. Therefore, the $50 \mathrm{~mm}$ length crystal was first evaluated. The idea was to try the longest crystal and measure the power out as a function of pump power, and if the crystal damaged (on the output side by the pump being back-converted), then try the $40 \mathrm{~mm}$ long crystal. The graph below shows the DFG output power as a function of the input pump power. Since the crystal didn't damage, the tuning had to be evaluated over the full range of wavelengths.

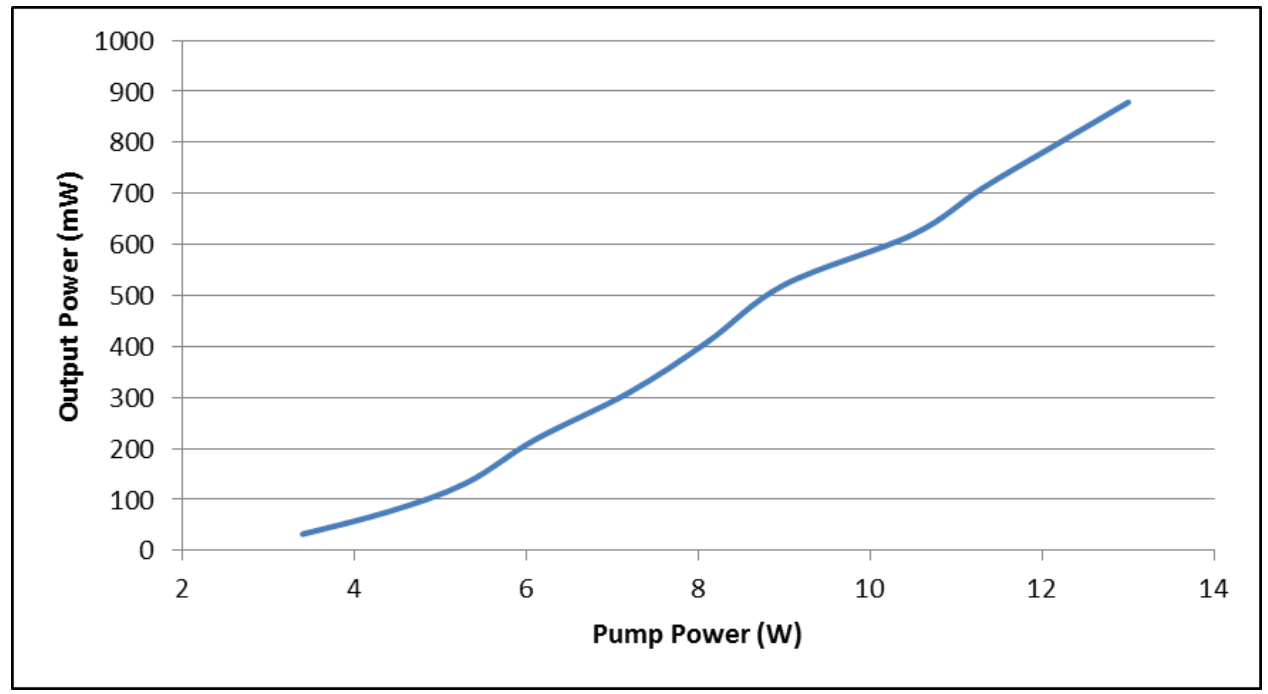

Figure 35: The power out of the DFG as a function of the pump power with a $50 \mathrm{~mm}$ long crystal.

Tuning is achieved by simultaneousley changing the seed wavelength and the input angle to the DFG crystal. Changing the seed wavelength is done by the high speed switch, as discussed earlier, while the input angle to the DFG is done by a galvonometer (galvo). The mirror mounted to the galvo motor is a custom optic. It is a fused silica substrate that is $3 \times 4 \times 1 \mathrm{~mm}$ thick with a dual high-reflecting coating for 
both $1064 \mathrm{~nm}$ and the seed range of $1530-1570 \mathrm{~nm}$. In order to tune through the full wavelength range, the face of the galvo mirror is relay-imaged to the center of the DFG crystal. The galvo was controlled by a National Instrument analog output device, which supplies the necessary input voltages to the galvo controller. For simplicity, the control voltage $-1.5 \mathrm{~V}-1.5 \mathrm{~V}$ was converted into galvo counts $(0-65535)$.

One of the requirements of this system is that it must tune within ten pulses (one milliseconds), which means the galvos must move and settle within that time frame. To measure this, a HeNe laser was reflected off the galvo mirror to a line array camera running at 45,000 frames per second. The galvo mirror was rotated while recording the location of the beam on the camera. The results of five galvo motors are shown below. As shown below, two motors (656 \& 658) didn't meet the requirement; fortunately, there are some adjustments on the controllers to optimize their performance.

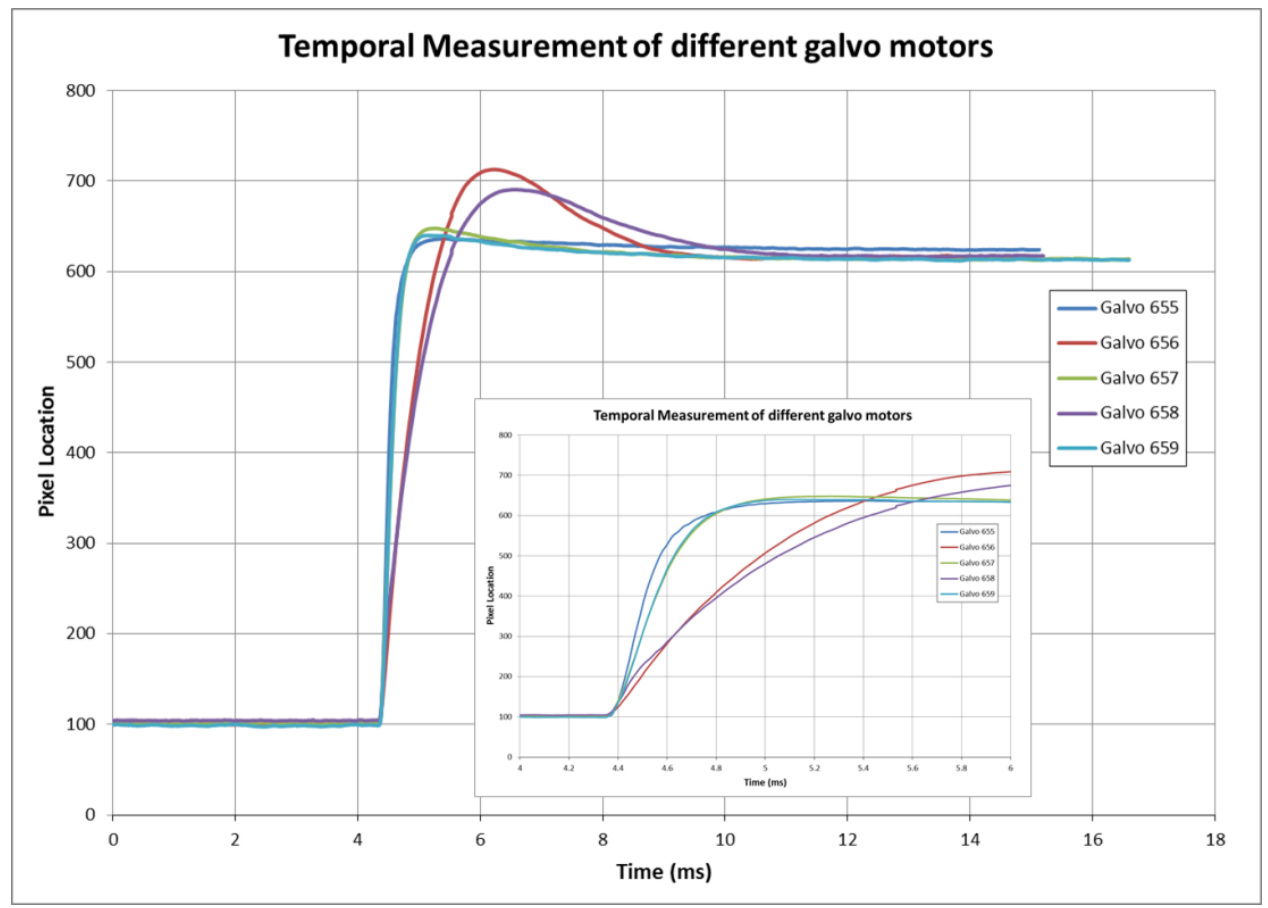

Figure 36: Temporal measurements of the galvo motors.

The following graph shows the power out of the DFG as a function of the seed wavelength, along with the galvo setting to optimize the phase matching. This data was collected with approximately 11 watts of pump power and a half watt of seed power. The seed wavelength was increased in $5 \mathrm{~nm}$ increments and the galvo setting was manually adjusted for maximum output power. The equation for the linear fit of the galvo setting (also plotted below) was used to determine the position of the galvo for the automated control system. 


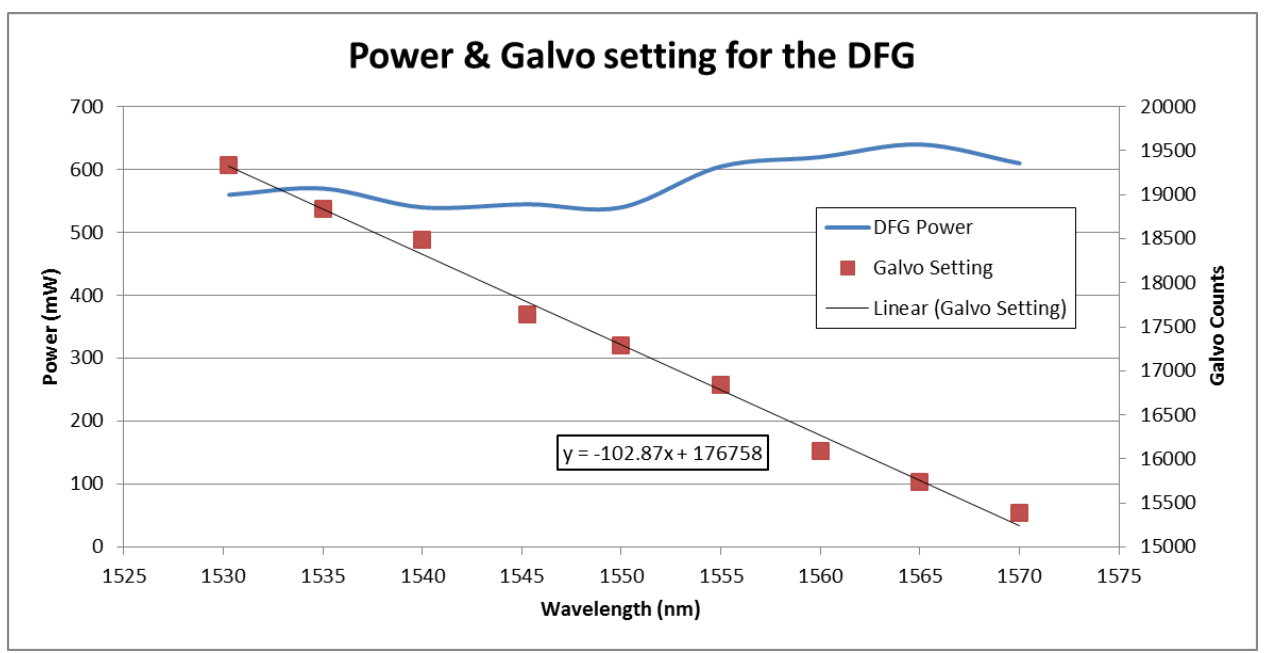

Figure 37: Shows the power out of the DFG across the full tuning range.

\section{Optical Parametric Amplifier (OPA):}

This is a process in which a weak laser beam at wavelength $\lambda_{s}$, the signal, is amplified by a nonlinear interaction involving a strong laser beam at a wavelength $\lambda_{p}$, the pump. To conserve energy, additional photons at a wavelength $\lambda_{i}$, the idler, is also simultaneously generated.

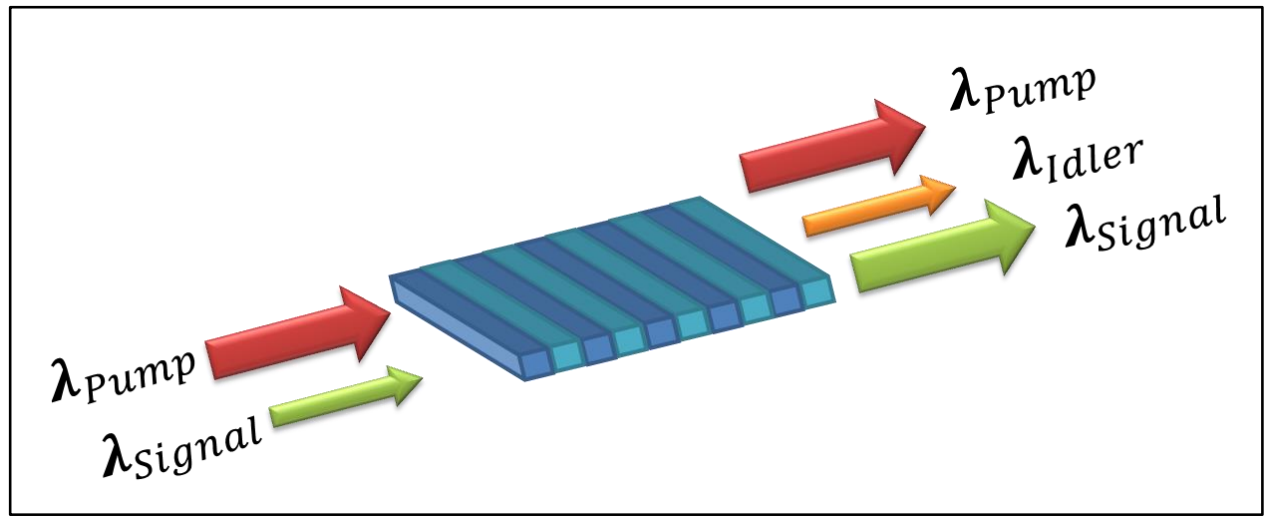

Figure 38: The diagram of the amplification process used.

In this case, the signal wavelength is produced in the DFG crystal which was then relay-imaged to the OPA crystal where it was combined with the rest of the pump beam and amplified. Since both the pump and the signal wavelengths are the same as in the DFG, the PPLN parameter (poling period \& angle) are also identical. The only change could possibly be in the length of the crystal, which is a function of the gain. The size of the pump beam at the OPA crystal is roughly 750 um (shown below). With about 65 watts of power and 20 nanosecond pulsewidth, the pump intensity is approximately $735 \mathrm{GW} / \mathrm{m}^{2}$. 


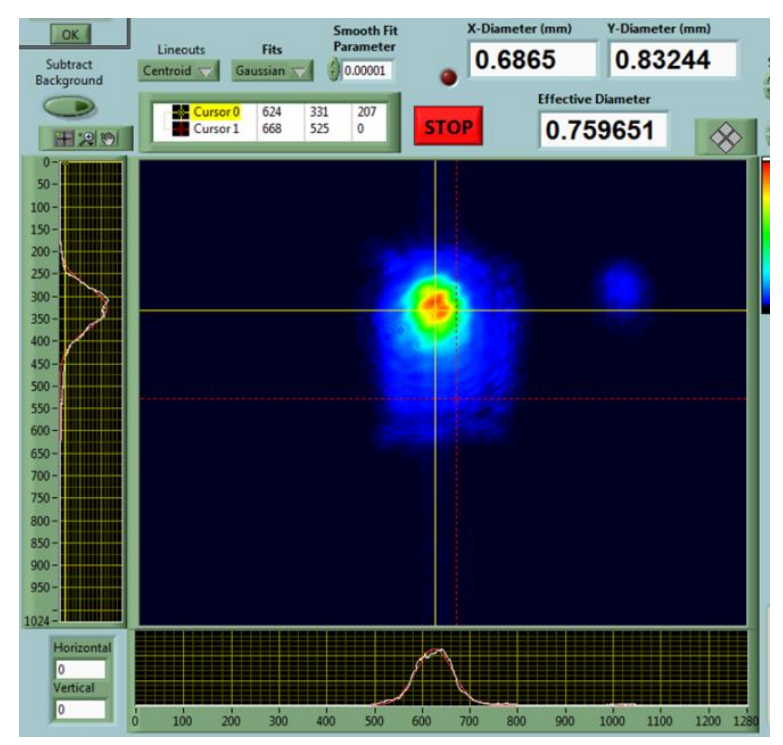

Figure 39: The beam size of the pump at the OPA crystal face.

Inserting this pump intensity into the parametric gain factor equation from the DFG section, the single pass gain for the OPA is shown in the graph below. Again, the different plots represent the gain at the ends of the required wavelength range.

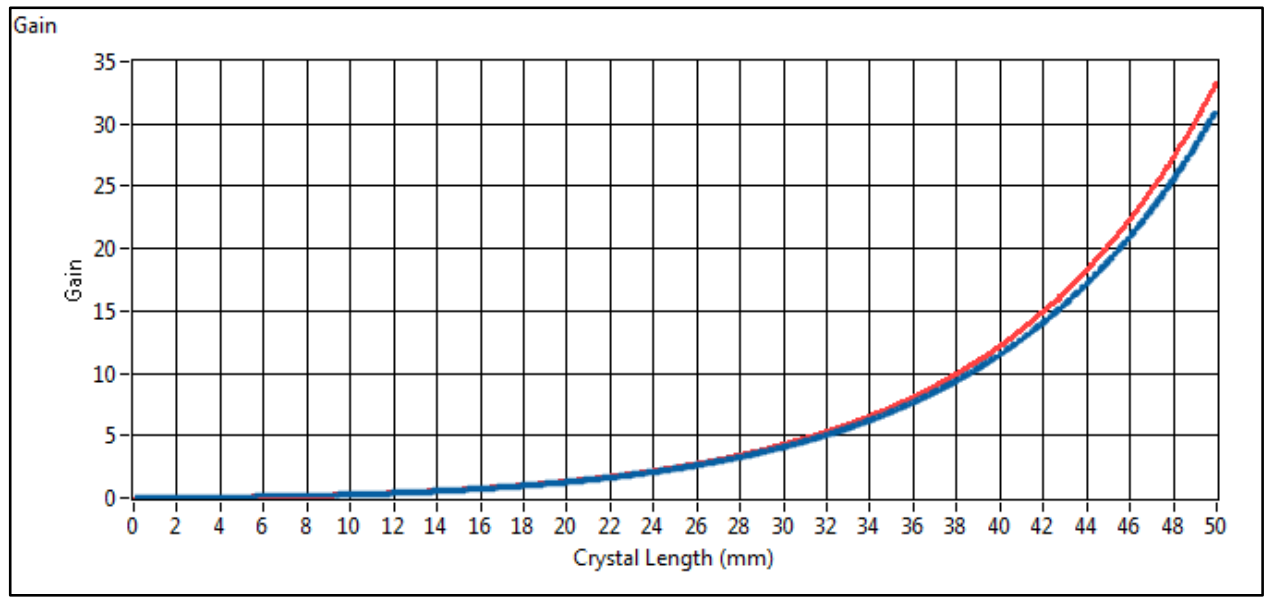

Figure 40: The gain calculation of the OPA as a function of crystal length.

Again, in reality, the crystal length for the OPA, like the DFG, was chosen empirically. The $50 \mathrm{~mm}$ long crystal was first tried with a reasonable pump diameter and measured the output. If the crystal was damaged, the $40 \mathrm{~mm}$ crystal would be tested. Fortunately the $50 \mathrm{~mm}$ crystal survived. The plot below shows the output power of the OPA versus the pump power at a single frequency. 


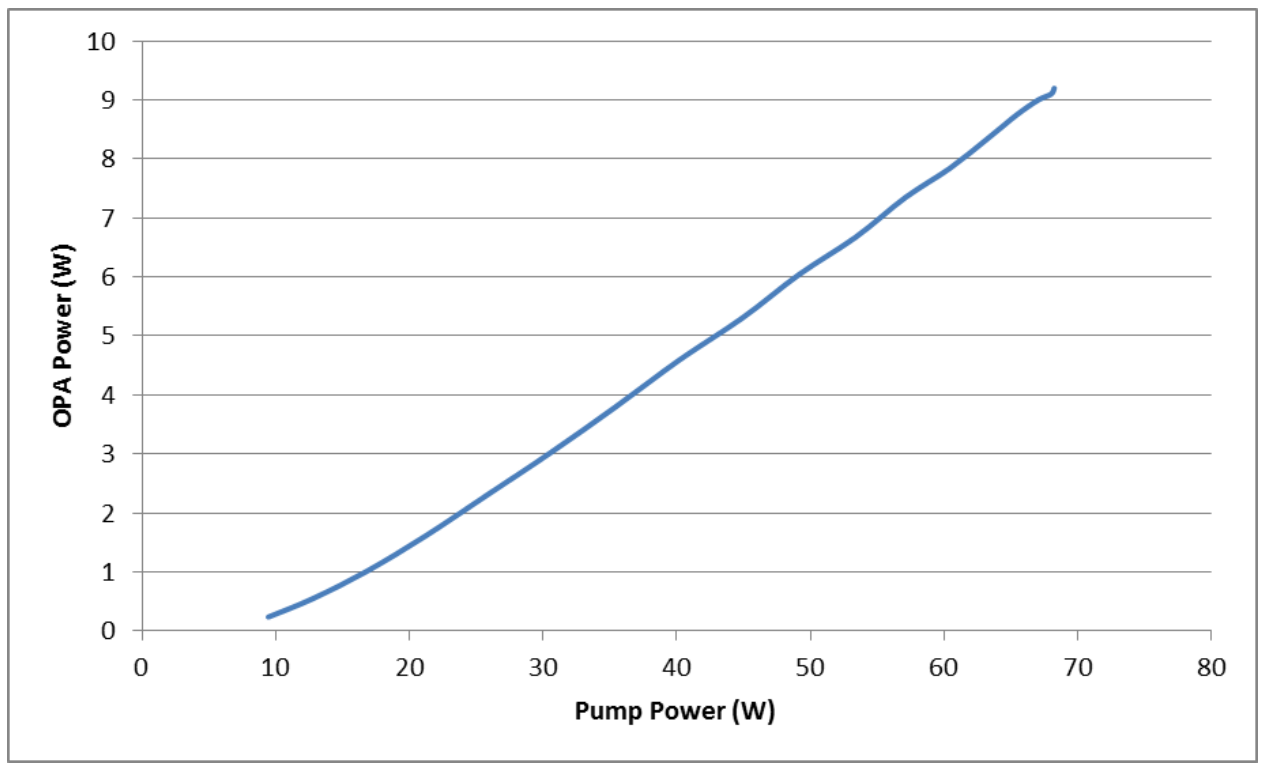

Figure 41: The power out of the OPA as a function of pump power.

Now that the crystal length has been determined, the tuning across the full range was verified. Tuning occurs by changing the angle of the pump beam, which is achieved by changing the angle of Galvo \#2. The mirror face on Galvo \#2 is relay-imaged to the center of the OPA crystal. If the power rolls off one edge of the range more than the other, it means that the pivot point (image plane) isn't centered in the crystal. The following graph shows the output power as a function of the DFG seed wavelength along with the galvo \#2 counts. The equation (shown on the graph) of the linear fit of the galvo count was used to determine the position of the galvo in the automated control system. The tuning was performed at low pump power as a precautionary measure to avoid damaging the crystal if phase matching was lost.

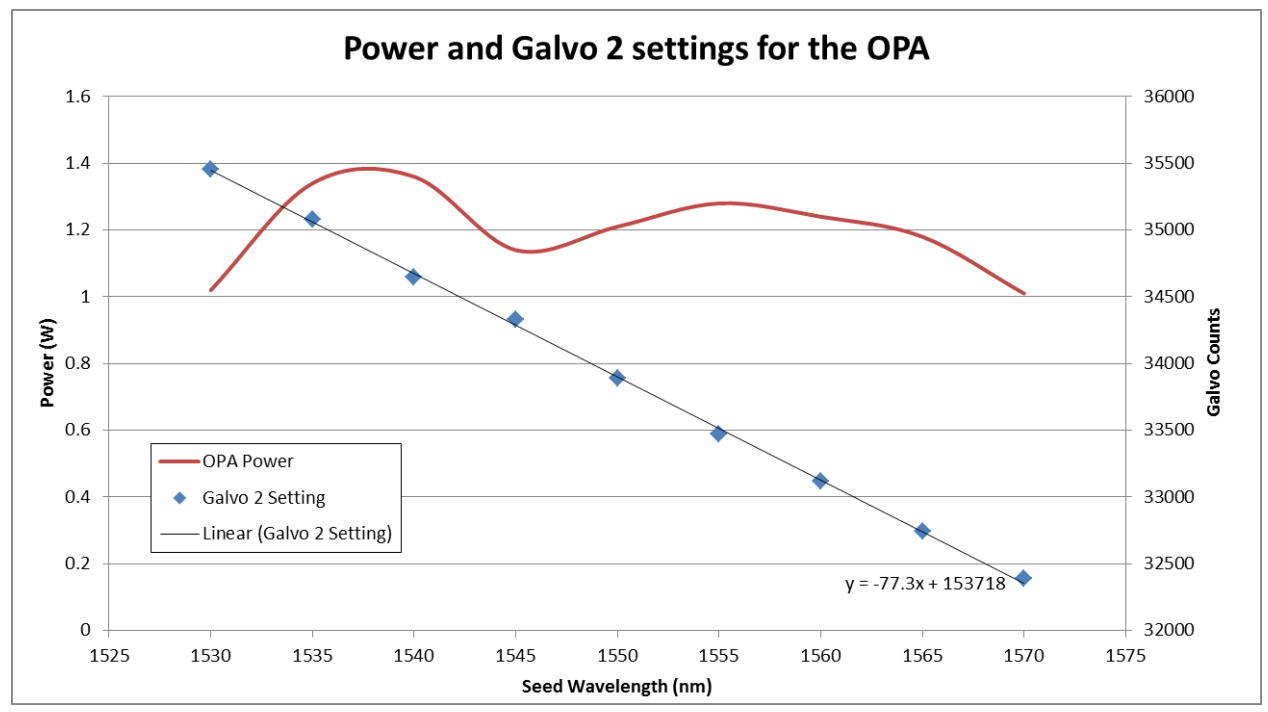

Figure 42: Shows the power out of the OPA (at low pump power) and the galvo 2 counts over the full tuning range. 
Galvo 3, which is located after the OPA, is the last actively controlled mirror used to correct any alignment error due to walk-off in the OPA crystal (in one axis only). In order to set this alignment, the laser was propagated several meters to a Coherent power meter while using the Coherent Labmax software (which contains Bulls eye view), which has the ability to measure the location of the beam relative to the center of the sensor. The seed wavelength was first set to $1530 \mathrm{~nm}$ and the laser was then aligned to the center of the sensor using mirrors after galvo 3 . Once set, the mirrors after galvo 3 were not touched. The seed wavelength was then changed to the next setting (along with galvo 1 and 2 to achieve phase-matching) and galvo 3 was manually adjusted until the beam was centered in the sensor. The graph below shows the galvo 3 counts as a function of the seed wavelength and the linear fit. This equation was entered into a configuration file that is used in the control system.
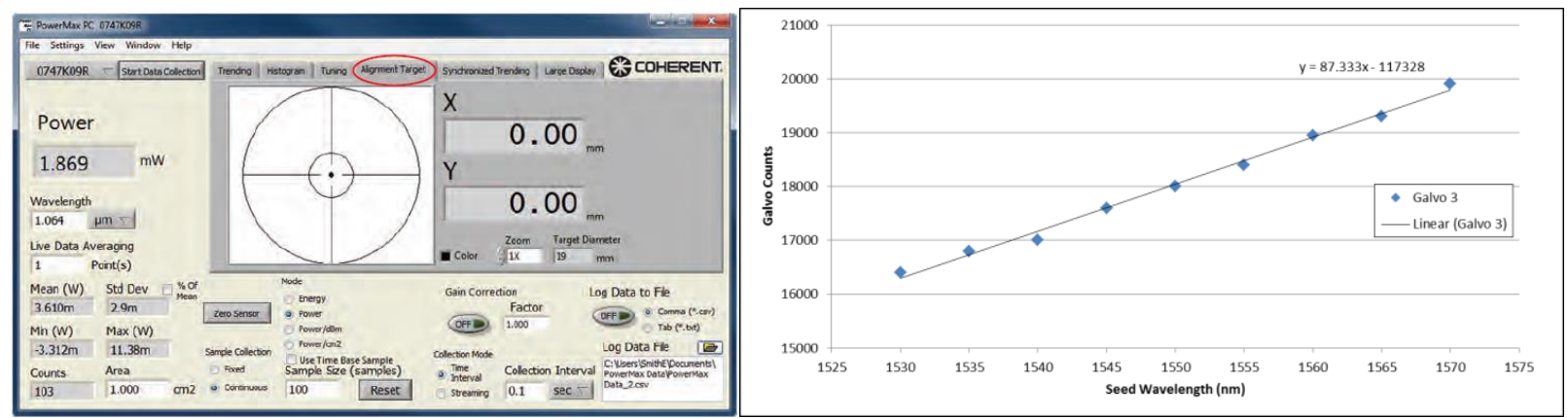

Figure 43: (a) Picture of the Coherent software used to get the galvo 3 (b) settings.

\section{Calibration Gas Cell:}

In order to accurately determine the output wavelength, both the seed wavelength and the pump wavelength need to be known. The seed wavelength can be easily measured using the Toptica wavelength meter, model WS7, IR-II. The manufacturers stated accuracy for this equipment is $40 \mathrm{MHz}$. To accurately determine the pump wavelength, the leakage light from a turning mirror of the output beam was aligned to the calibration gas cell system. A diagram of the gas cell system is shown below and the equation to the absorption is:

$$
\alpha=-\ln \left(\frac{T}{I}\right)=-\ln \left(\frac{P D_{\text {sig }}}{P D_{\text {ref }}}\right)
$$

where $P D_{\text {sig }}$ and $P D_{\text {ref }}$ are the voltages measured from the signal and reference photodiodes. 


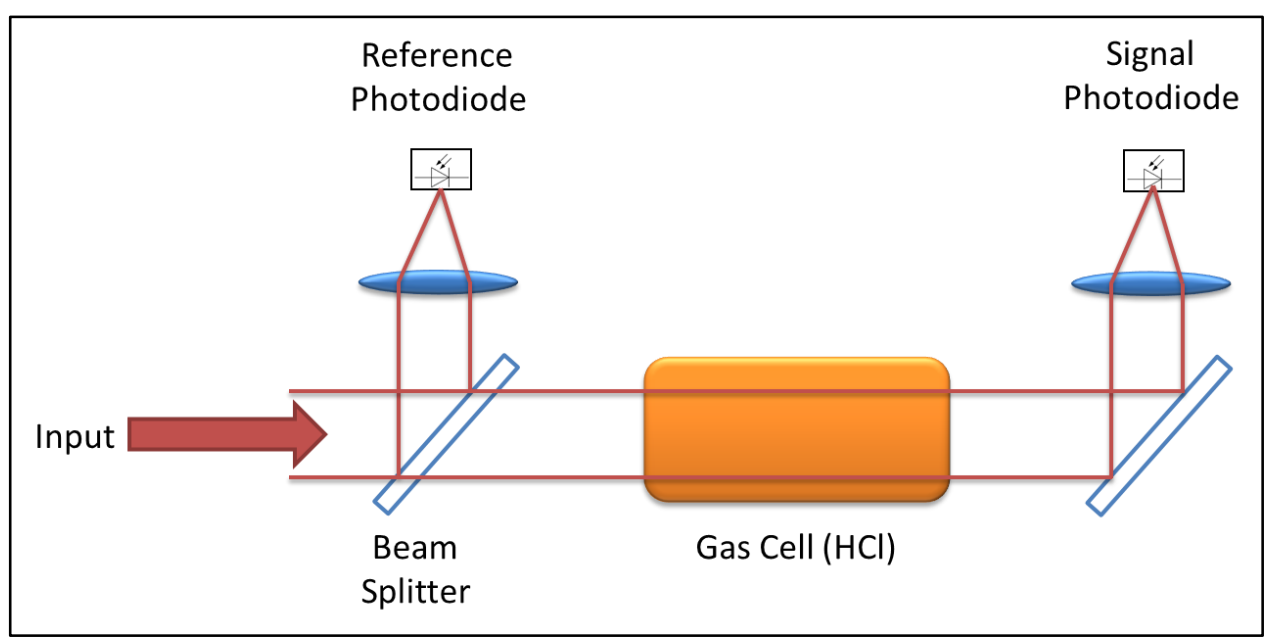

Figure 44: A diagram of the calibration gas cell system.

The gas used in the calibration gas cell is hydrogen chloride $(\mathrm{HCl})$, which has a very well-known absorption spectrum in the mid-wave range. To determine the pump wavelength, a seed source was scanned over two HCL features, 3.3746 and 3.3772 micron, as the voltage from both the reference and signal photodiodes were measured. The seed wavelength was accurately measured using the Toptica wavelength meter and the peaks were aligned using the equation $\frac{1}{\lambda_{i}}=\frac{1}{\lambda_{p}}-\frac{1}{\lambda_{s}}$. Using a pump wavelength of 1064.3848 nanometers, the measured absorption peaks line up with the $\mathrm{HCl}$ absorption peaks.

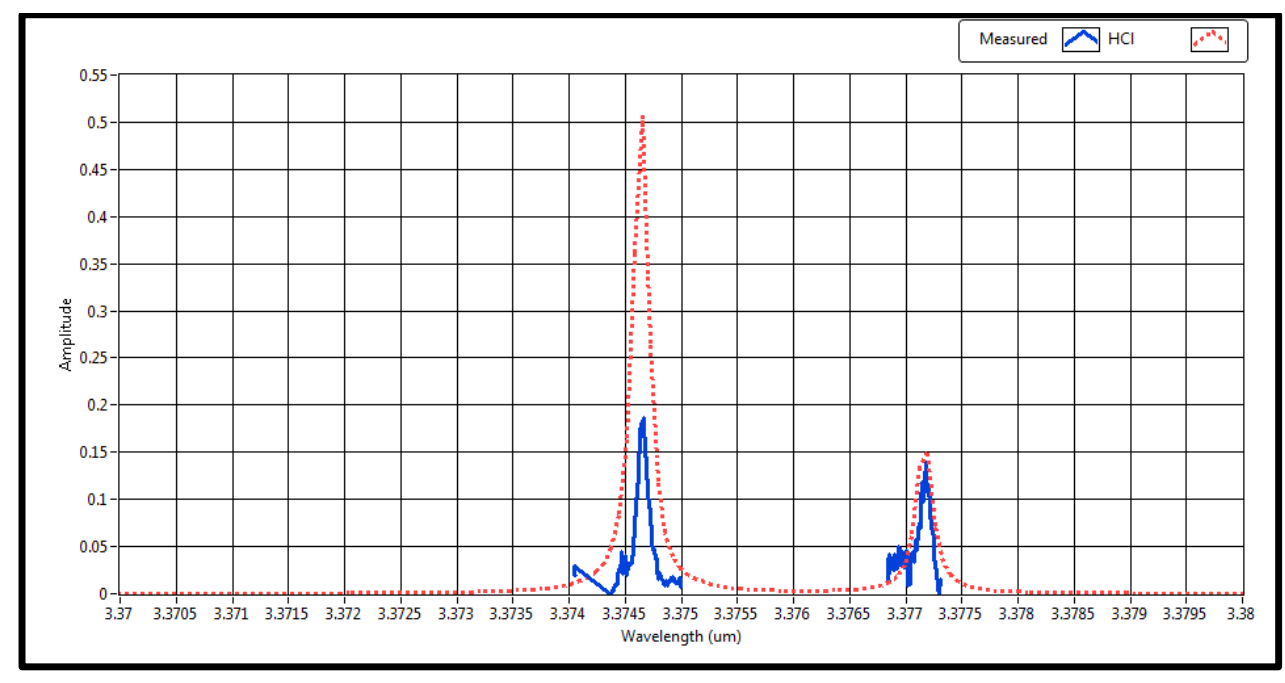

Figure 45: The calibration gas cell fit to $\mathrm{HCl}$ absorption peak. 


\section{Receiver:}

The receiver incorporates a cryogenically-cooled 8x8 Mercury Cadmium Telluride (HgCdTe) avalanche photo-diode (APD). A sketch of the basic concept is shown in the figure below.

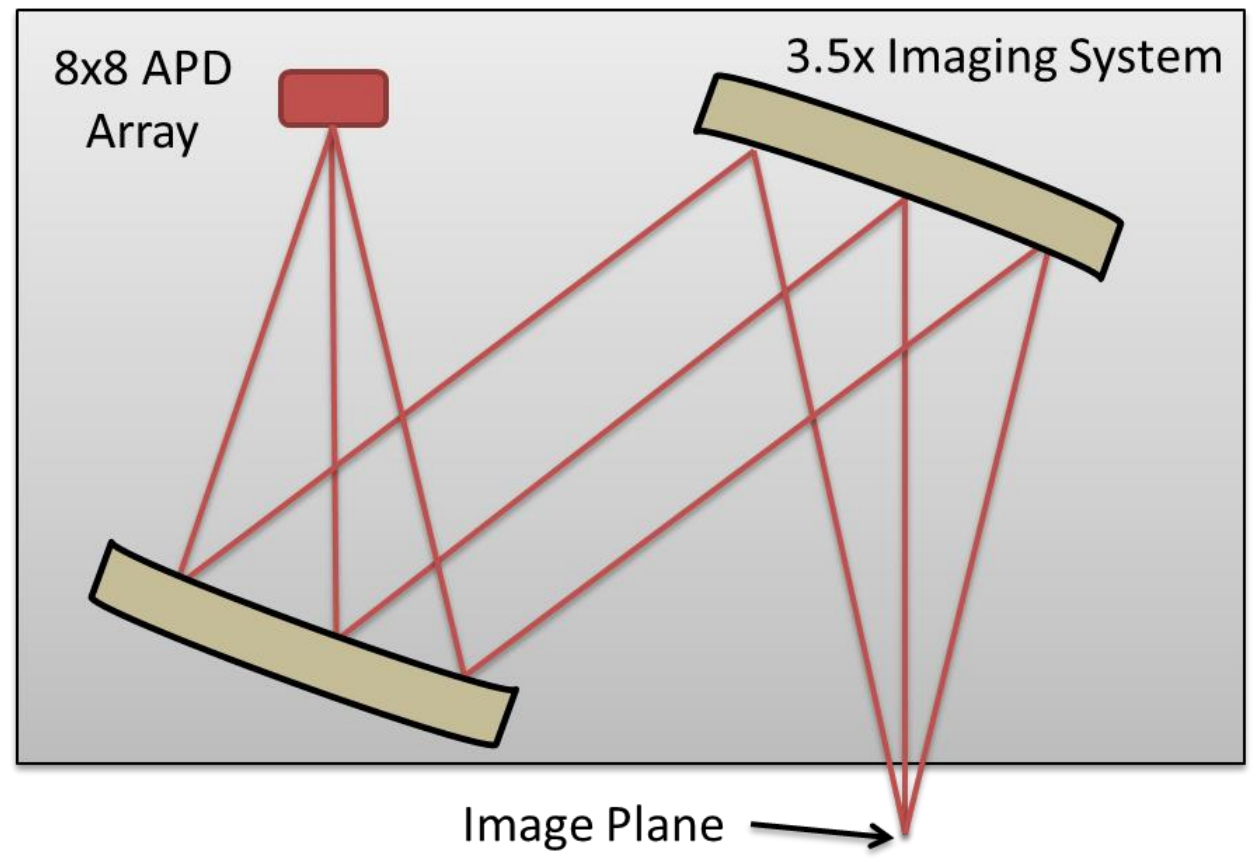

Figure 46: A sketch of the basic concept of the receiver.

The detector pixel size is 70 microns square with an internal magnification of 3.5x, therefore making the effective pixel size at the image place $\mathbf{2 4 0}$ microns. The receiving optical system (external to the receiver) consists of the 16 inch gimbal head mirror, a 10 inch parabolic mirror with a focal length of 60 inches, and a 6 inch flat turning mirror. This creates an effective pixel size of $0.16 \mathrm{mRad}(160 \mathrm{~mm}$ at $1 \mathrm{~km}$ range). The receiver operates in a peak-detection mode and is capable of running at $20 \mathrm{kHz}$. Since the transmitter is running at $10 \mathrm{kHz}$, it means that every laser frame also has a background frame that can easily be subtracted from the signal pulse.

The communication to and from the receiver was done through a serial Front Panel Data Port (sFPDP) PCle card from Curtiss-Wright. The Application Program Interface (API) and software were also purchased with this card. This gave us the ability to write our own software to interface directly with the card. In order to do this, a C-code interface was developed and complied into a DLL which was then called from LabVIEW.

\section{Packaging:}

The components were mounted to a 4 foot by 5 foot enclosed breadboard that was mounted to a hydraulic lift table. The lift table has a $\mathbf{3 0 0 0}$ pound maximum lift capacity with maximum lift height of approximately 34 inches. This allowed the system to be raised above any obstructions. The lift table 
was mounted onto a 4 by 6 foot trailer which made it possible to be moved by two people. Camper jacks were installed on the enclosure to provide stability and safety when the system is lifted.

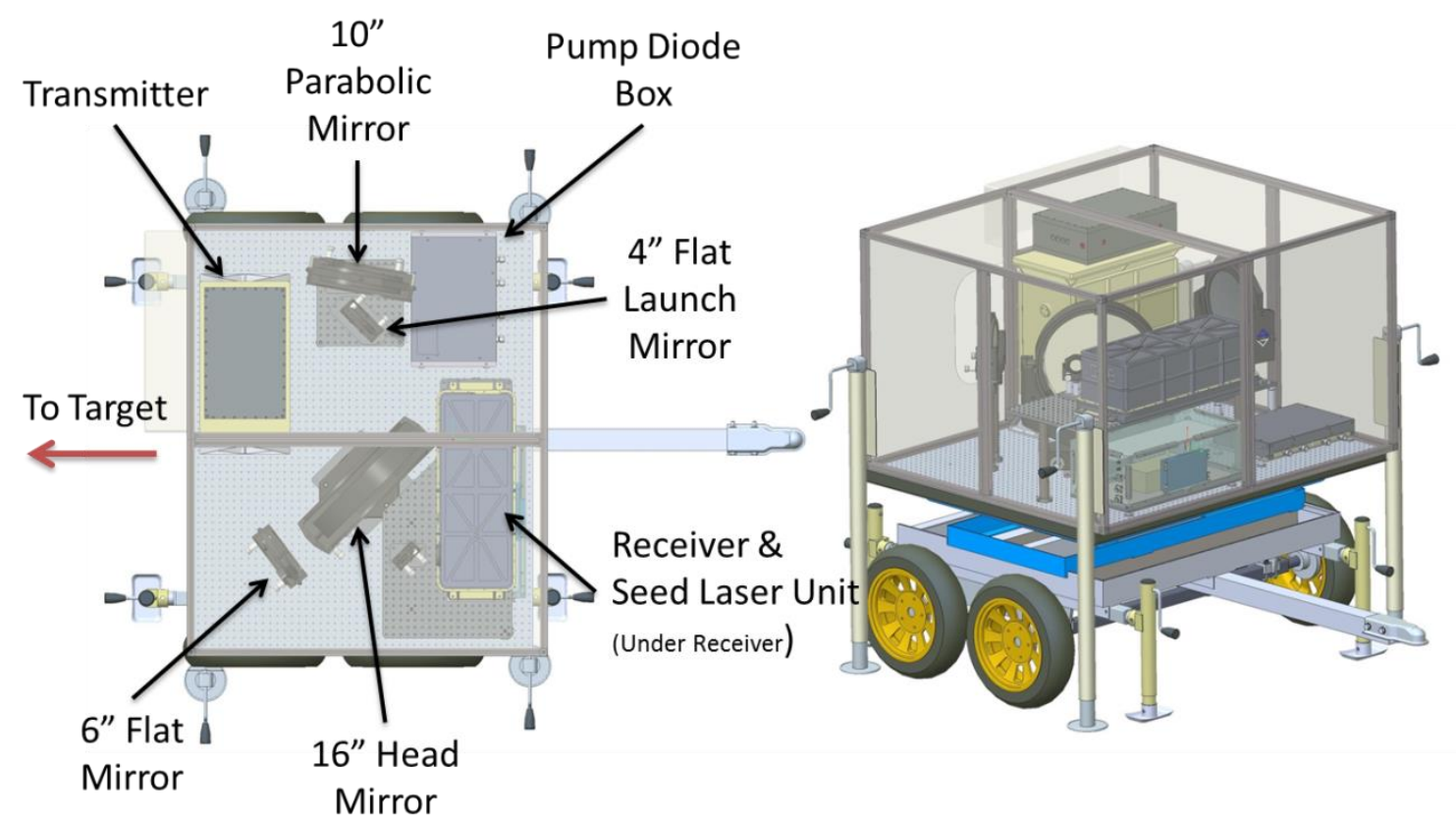

Figure 47: The mechanical layout of the ground based remote sensing system.

With the system assembled, the alignment of the transmitter to the receiver was necessary. To do this, an $\mathrm{F}^{\#} 4$ collimator with a 6" aperture was placed approximately two meters from the output of the ground cart. At low power, the transmitter was aligned to the collimator using the 4" flat launch mirror and the 16" head mirror. Temperature sensitive liquid crystal paper was used to make the alignment of the transmitter easier. The receiver was aligned to the collimator by placing a halogen lamp at the pinhole location of the collimator. The light from the lamp was then aligned to the receiver window by adjusting the 10 " parabolic mirror and the 6 " flat mirror. The focus was also set by adjusting the distance from the 6" mirror to the receiver window. The figure below shows the model of the transmitting and receiving optics using the optical design software Zemax. Since the halogen lamp produces a lot of heat, the receiver can easily see the lamp when it is properly aligned. 


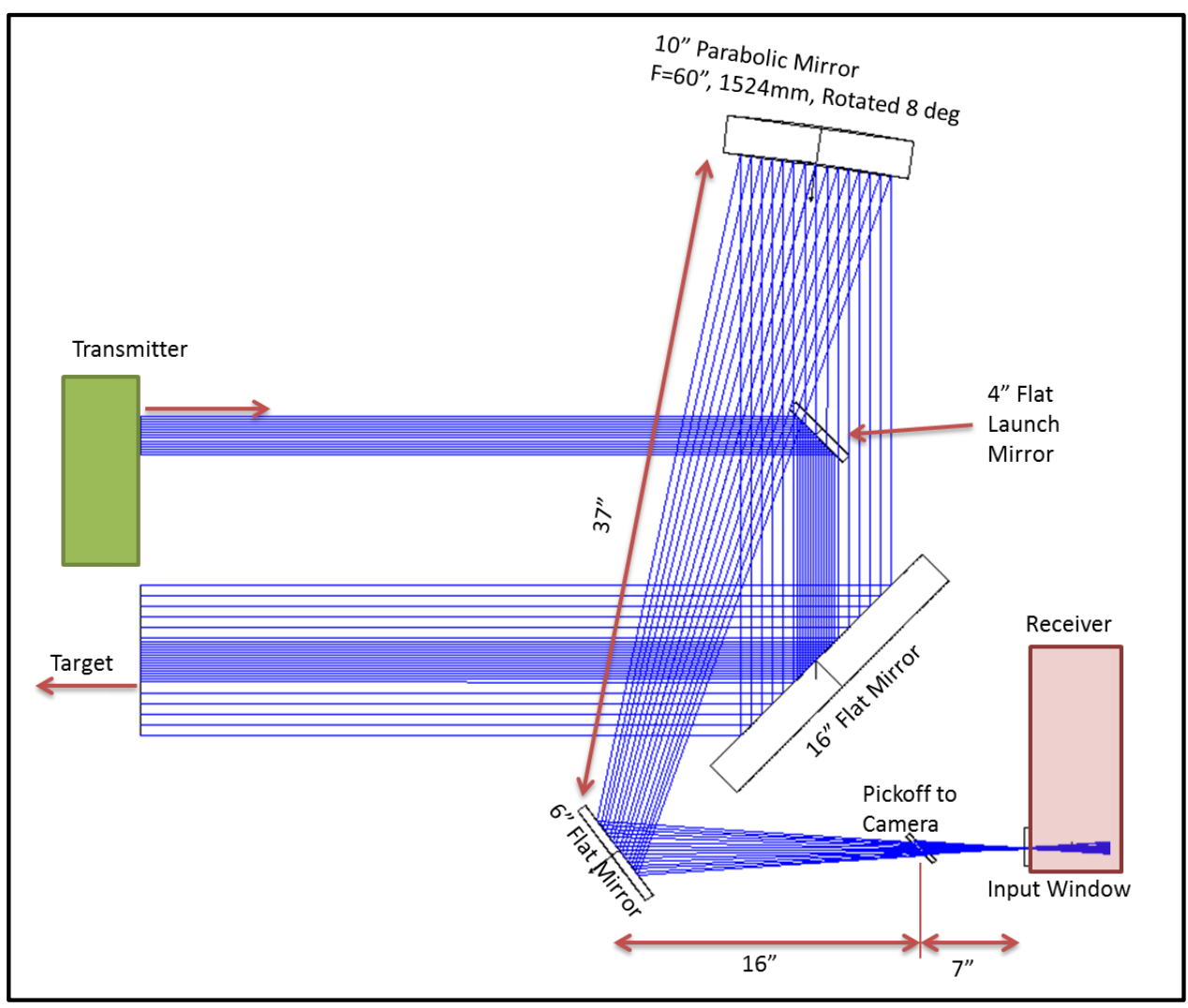

Figure 48: The Zemax model of the launch and receive optics.

With both the transmitter and receiver aligned to the collimator, the next step was to try to detect the transmitter light on the receiver. This was done by seeding the transmitter with only one source and adjusting the trigger delay of the receiver.

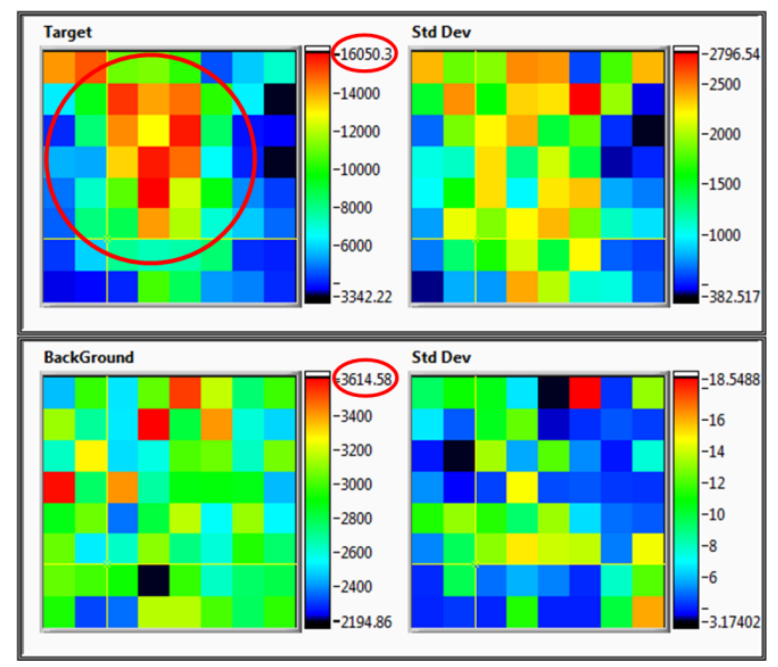

Figure 49: The first image of the transmitted beam collected by the receiver. 


\section{Control System:}

The system was controlled by a single computer running 64-bit version of LabVIEW 2014. The software is an event-based architecture, meaning the software waits for the user to provide the event. The computer used three serial ports; one for the waveplate in the transmitter to control pump power, one to control the picomotor controller for the pointing \& centering mirror mounts, and one to communicate to the four pump diode power supplies. There were three USB CCD cameras from ThorLabs; two in the transmitter for pointing \& centering, and one that was co-aligned with the receiver in order to give us a visual on the target. A four-slot National Instrument Compact DAQ (Data Acquisition) chassis (cDAQ-9174) with two analog 16-channel output modules, a 16-channel analog input module, and a 4-channel bidirectional digital input/output module, was used to monitor and/or control different subsystems. The digital input/output module was used for synchronization of the analog input and output modules with the master clock. The head mirror was controlled by a second LabVIEW program running on the same computer that communicated with the chassis through an Ethernet port.

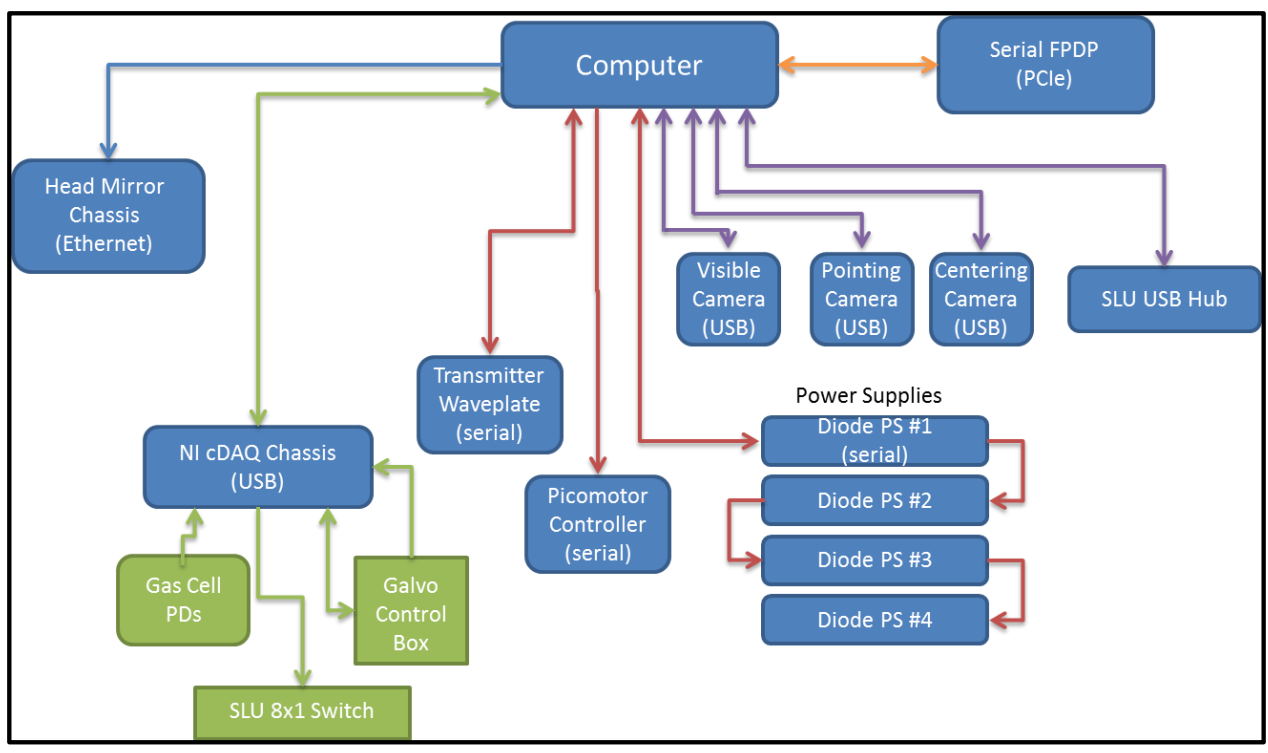

Figure 50: Block diagram of the control points.

\section{Operations \& Results:}

Once the system is up and ready to start collecting data, the seed wavelengths need to be set, along with how many pulses at each wavelength and the order of the wavelength. This information is set in the Line Sequencing Table (LST) and the Line Sequencing Description (LSD), which will be talked about later. Determining what wavelengths to use depends on the target material and the environment in which the test is occurring. In this first example, the target material is butane gas in a laboratory setting 
where atmospheric absorption is not a concern. This graph shows the absorption plot of butane at 1 part per million-meter along with the test locations that were sampled.

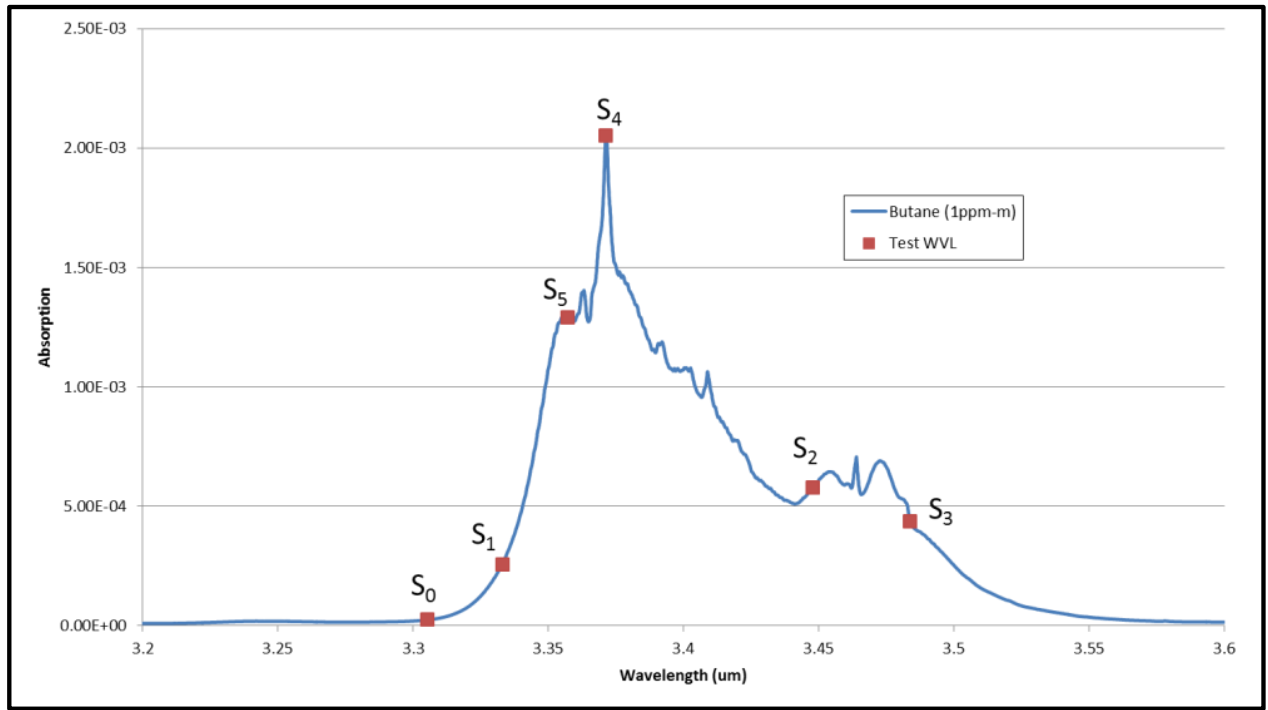

Figure 51: The absorption spectrum of butane with the test locations.

Once the wavelength of the seeds (test locations) is set, the LST and LSD need to be produced. The Line Sequencing Description (LSD) contains information that is important when analyzing the data; information like the MOPA wavelength, the source wavelengths, the number of sources used, the total number of pulses, the order of the sources, and a flag (which will be discussed later). This information is part of the header of each data collect file. That way there is no discrepancy with what settings were used. The Line Sequencing Table is a table that contains the voltage settings for the high-speed switch and the three galvo settings for each pulse. To create the LST and LSD, a LabVIEW program was written that, with the given MOPA and Source wavelengths, produces the required information for the LST and LSD. This program allows the user to select a number of different options, like the number of pulses per source and the number of galvo positions. When finished, the program creates the LSD for the data collect file and the LST. The LST is used by the automated control system during the data collect to set the position of the switch and galvos.
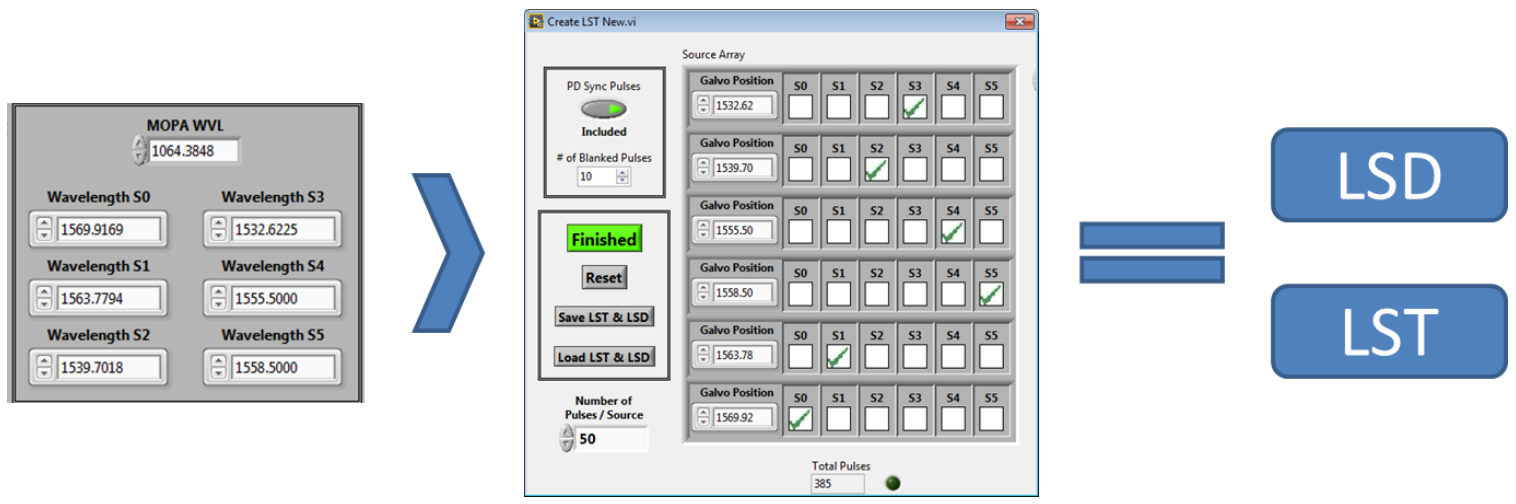

Figure 52: The program used to create the Line Sequencing Description and Table. 
For the example shown above, each source has a separate galvo setting and will stay on that source for 50 pulses. There are 10 pulses that are "blanked" between each of the galvo settings. These blanked pulses are flagged in the LSD as being "no good" because these are the pulses where the galvos are moving and, therefore, the output might be unstable. The analysis software will discard the pulses that are flagged. The "PD Sync Pulses" included mean that there are 25 pulses added to the beginning of the LSD that are not properly seeded. Since these pulses are not seeded properly, the power will be significantly lower than the seeded pulses, and will be used as a tool in the analysis software to synchronize two different data files.

Now that the LST and LSD are created, data collection can be started. Once the "Start Collection" button has been activated, the computer creates two unique files. The first file contains the data collected from the receiver while the second file is the voltage measured from the reference photodiode from the calibration gas cell. Once the files are created, the computer then prompts the user if they want to open the laser shutter. When the answer is received, the LST is loaded into the sequencing system. For every trigger pulse, this system reads a single row of the LST and sets the appropriate voltage. When the sequencing system reaches the end of the LST it starts over from the beginning and will repeat until the user stops the data collect. When it is stopped, the computer closes the shutter and closes the data files. For the example above, the transmitter and receiver were aligned to the SORL collimator approximately two meters from the output of the cart. This data collect lasted around 45 seconds, in which butane was released (from a lighter) twice, for approximately 2 seconds. The gas was released at the location of the pinhole of the collimator.

Now that the data has been collected, it is time for the analysis. Depending on the length of the data collect, the file could be several gigabits. The first step in the analysis is to separate the good pulses from the transition pulses. This is done by using the flags in the LSD and correlating with the pulses in the data file and photodiode file. The plot below shows the photodiode voltage over two full LSD. The plot also displays the source order and the flag. For the flag, a 1 indicates a transition, or sync, pulse which is not used in the analysis, while a 0 signifies a good pulse. 


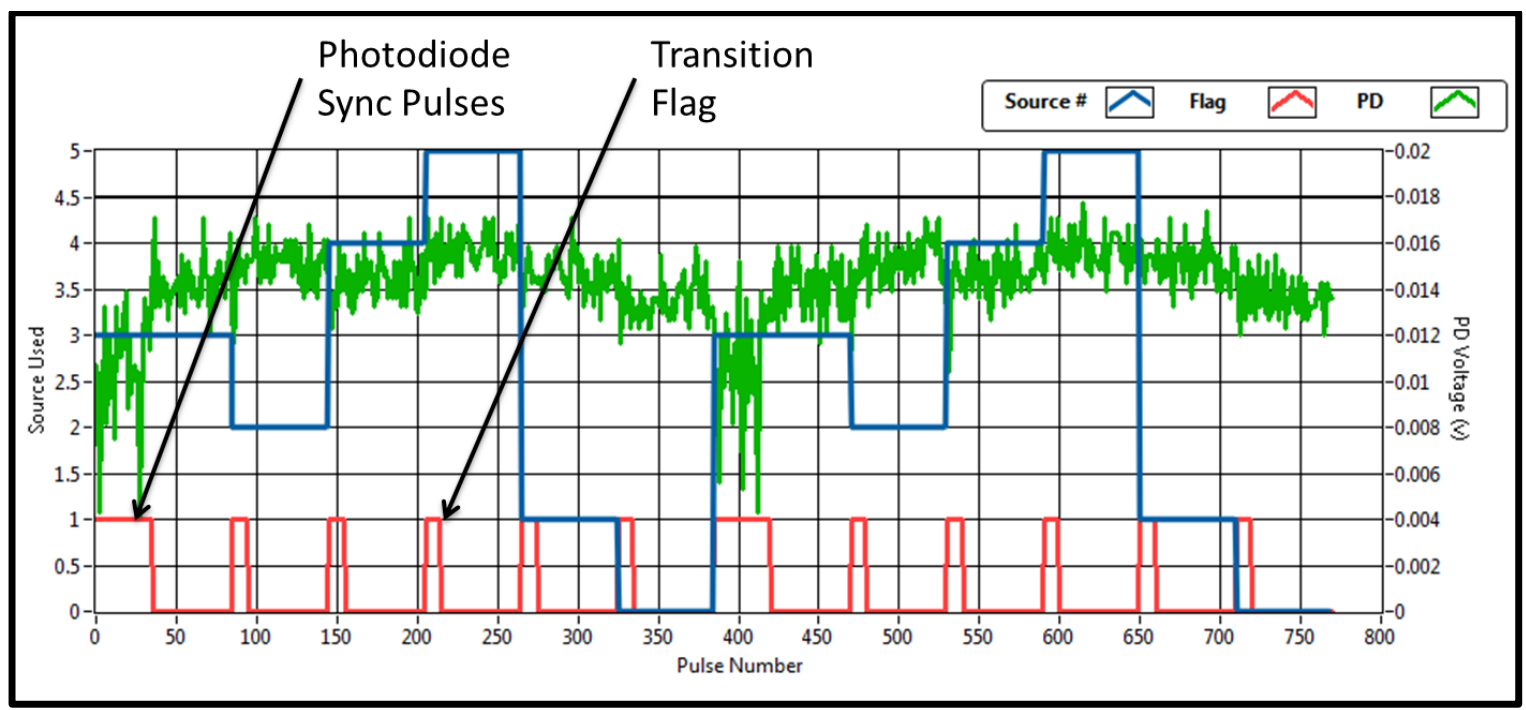

Figure 53: Plot showing two complete LSDs of the photodiode which indicate the flagged pulses and the source that is used for each pulse.

The next step in the process is to subtract the background frame from the signal frame collected from the receiver. As stated in the receiver section, the receiver returns two frames for every trigger pulse one background (no laser pulse), and one signal (with laser pulse). This subtraction is pixel to pixel for every signal frame. The next step is to average the returns from each source in a single LSD. In the example above, there were 50 pulses for each source per LSD. With each pulse, the receiver collects one signal frame that consists of 64 points ( $8 \times 8$ array), those points are then averaged together over the fifty pulses which then results in six 64-point arrays per every LSD. The graphic below displays the average returns for each source over one LSD (with no butane gas present).

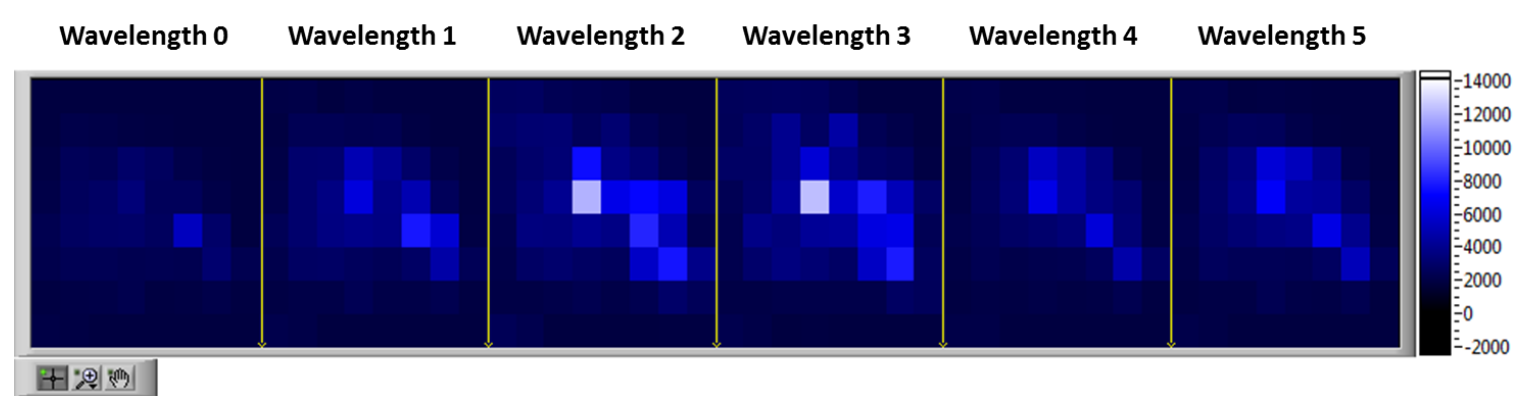

Figure 54: The average returns for each source over an entire LSD.

The final step in the analysis process is to calculate the concentration path-length of the target gas (butane). To perform this calculation, the equations from the differential absorption LIDAR (DIAL) technique are used.

$$
\begin{gathered}
\vec{s}_{i}=\frac{1}{2} \ln \left(\frac{\rho_{i+1}}{\rho_{i}}\right) \\
K_{i, l}=\alpha_{i}^{l}-\alpha_{i+1}^{l}
\end{gathered}
$$




$$
C L=\frac{K^{T} \vec{s}}{K^{T} \mathrm{~K}}
$$

The absorption at the sampled wavelengths $\alpha_{i}^{l}$ is given from the graph in Figure 51 and is shown in the table below.

\begin{tabular}{|c|c|}
\hline Test WVL & Absorption \\
\hline 3.30542 & $2.3887 \mathrm{E}-05$ \\
\hline 3.33296 & 0.00025711 \\
\hline 3.44788 & 0.00057915 \\
\hline 3.48391 & 0.00043612 \\
3.37121 & 0.002053 \\
3.35720 & 0.0012886 \\
\hline
\end{tabular}

Table 2: The absorption of butane at the location of the test wavelengths.

To determine the LIDAR return $\rho_{i}$ for each of the sampled wavelengths, the pixel amplitude was spatially averaged over the full or partial $8 \times 8$ array. In this case, the entire $8 \times 8$ array was used, except for the four corners (total of 60 pixels), and it was also temporally averaged over a half second. Since there were 385 pulses per LSD at a rep-rate of $10 \mathrm{kHz}$, half a second equals 13 LSDs that the amplitude was averaged over. The plot of the amplitudes for each wavelength that are both spatial averaged over the 60 pixels and temporal averaged of approximately a half second is shown below for the full collection.

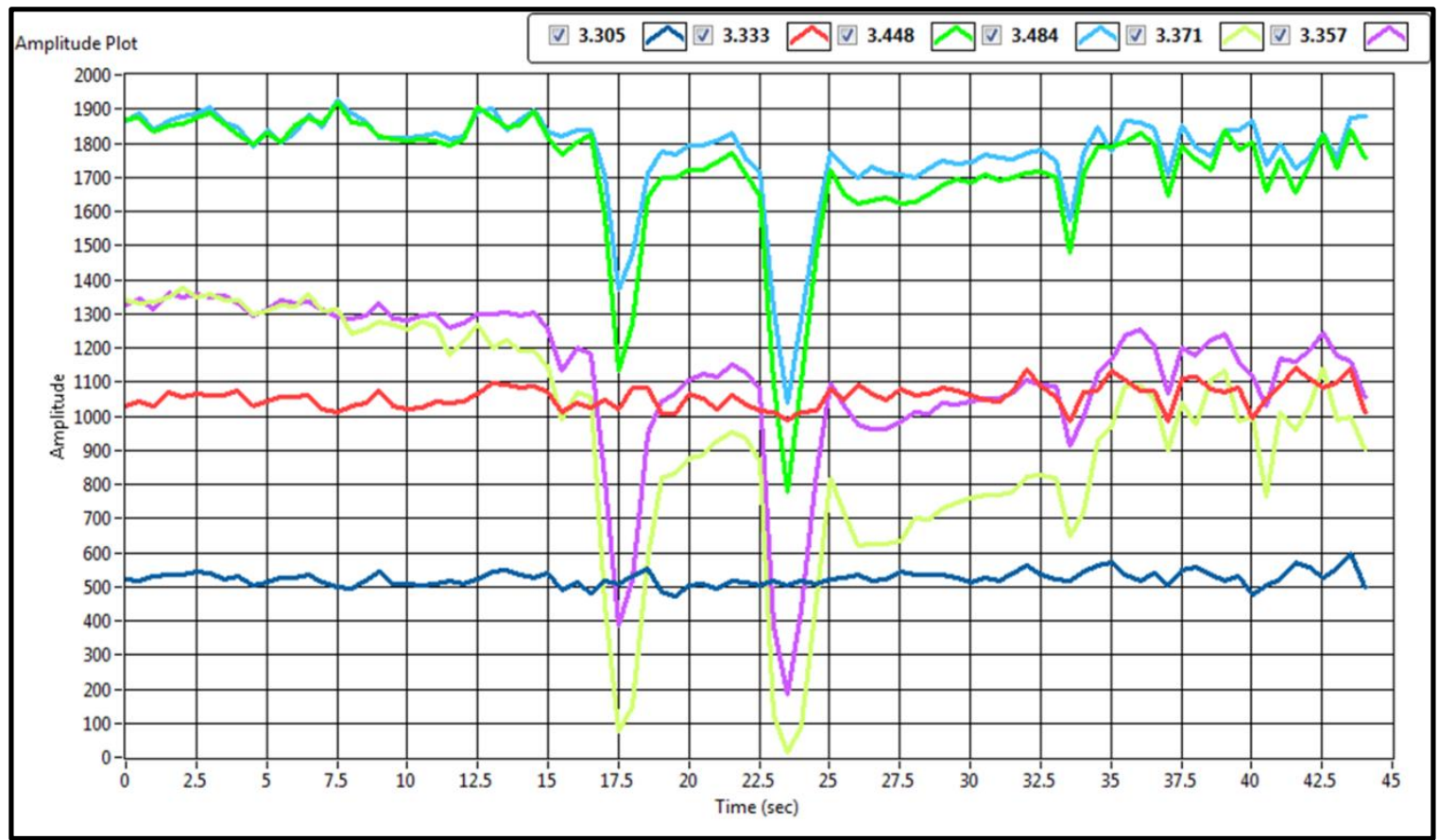

Figure 55: The spatial and temporal averaged amplitude for each of the test wavelengths. 
It is obvious when the butane gas was turned on ( $17.5 \& 24$ seconds). The graphic below shows the return of each wavelength averaged over the LSD with the butane gas present. Notice the drop in intensity for both wavelength 4 and wavelength 5 when compared to the intensity in Figure 54 .

$\begin{array}{llllll}\text { Wavelength } 0 & \text { Wavelength } 1 & \text { Wavelength } 2 & \text { Wavelength } 3 & \text { Wavelength } 4 & \text { Wavelength } 5\end{array}$

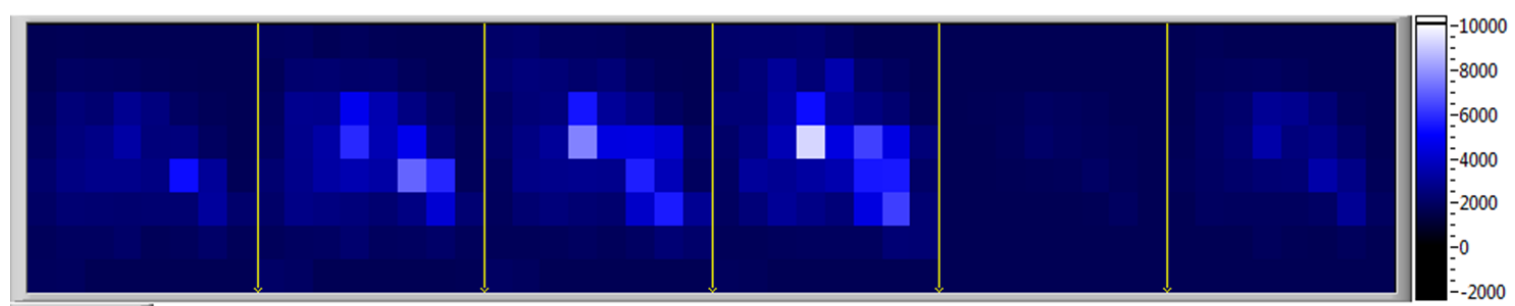

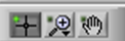

Figure 56: The averaged return for each source when butane gas is present.

The graph below shows the calculated concentration path-length of butane gas over the full data collection. Again this collection was done under ideal conditions.

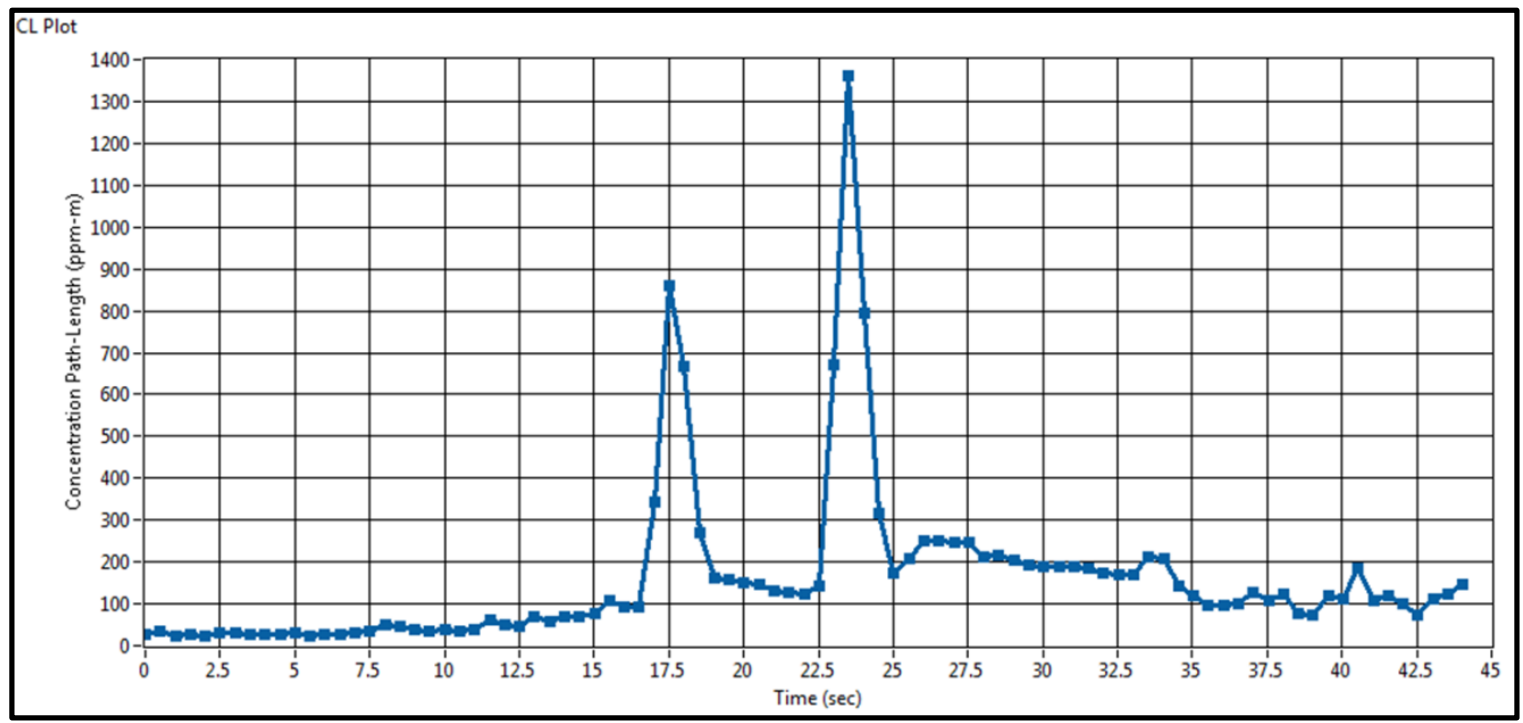

Figure 57: The concentration path-length calculation averaged both spatially and temporally.

In real world conditions, factors like atmospheric absorption must be considered when selecting the sampling wavelengths. As shown in the graph below, the location of the absorption peak of butane falls in an area where the atmospheric transmission is very poor. As a result, the test wavelength locations in the example above were moved to areas where the atmospheric transmission was better. 


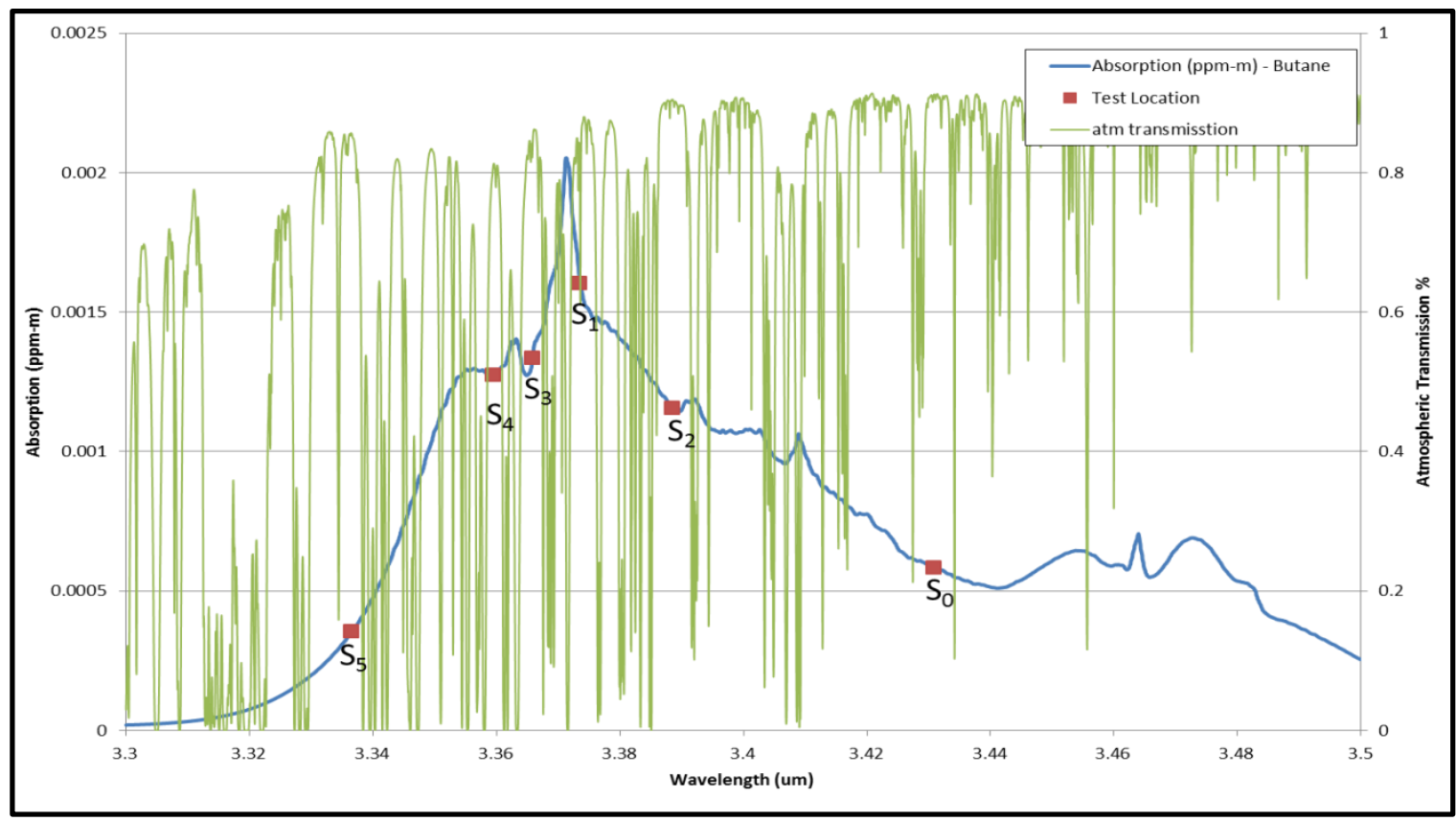

Figure 58: A plot showing the absorption of butane along with the atmospheric transmission with the test locations for an outside data collect.

For this data collect, the target was approximately 200 meters from the transmitter/receiver. The collection lasted about six minutes, during which the head mirror moved between two positions, "Off" target and "On" target as shown in the diagram below. The collection started in the "Off position then moved to the "On" position. This was repeated 4 times during the collection and ended in the "Off" position. The first and last "On" sequence the butane gas was not released, while the second and third "On" sequence the butane was released.

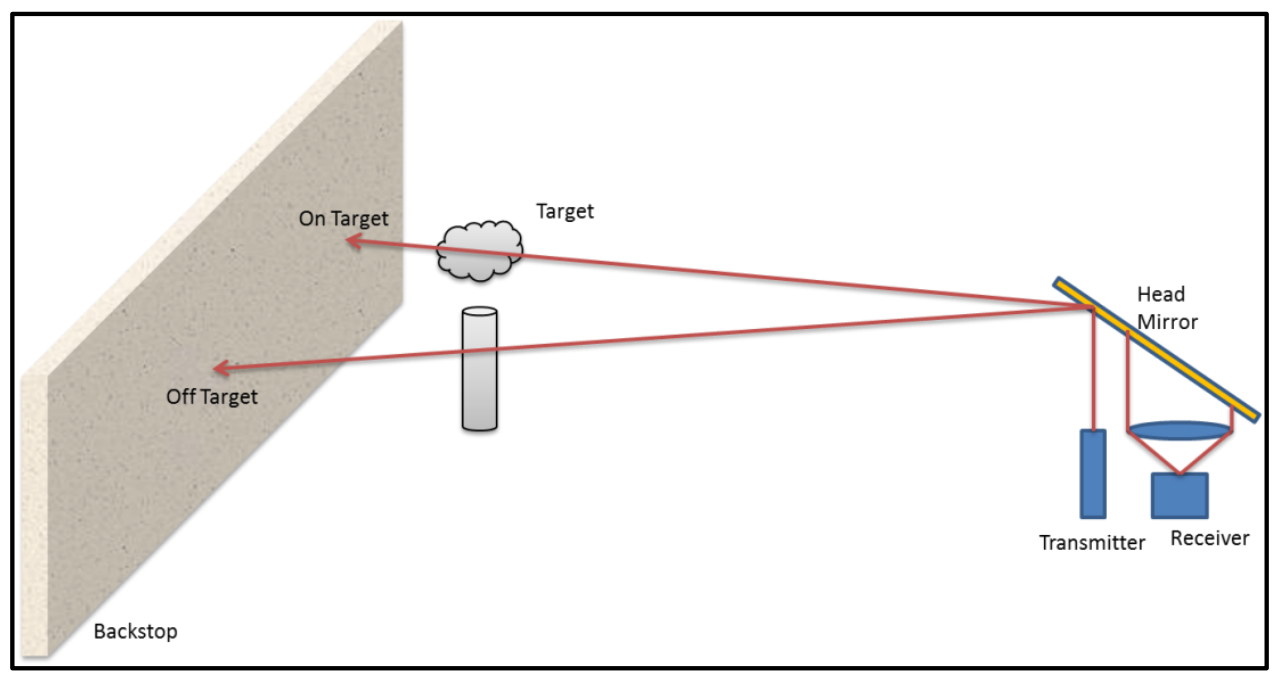

Figure 59: Diagram of the outdoor data collect.

The LSD for this collection was very similar to the first example; each source had 50 pulses with 10 transition pulses between sources, and 25 sync pulses, which make for a total of 385 pulses in the LSD. 
For the analysis, the return was spatially averaged for the full 8x8 array, except for the corners, but was temporally averaged over two seconds which is 52 LSDs. The plot below shows the averaged amplitudes for each sampled wavelength in the "ON" and "OFF" target positions.

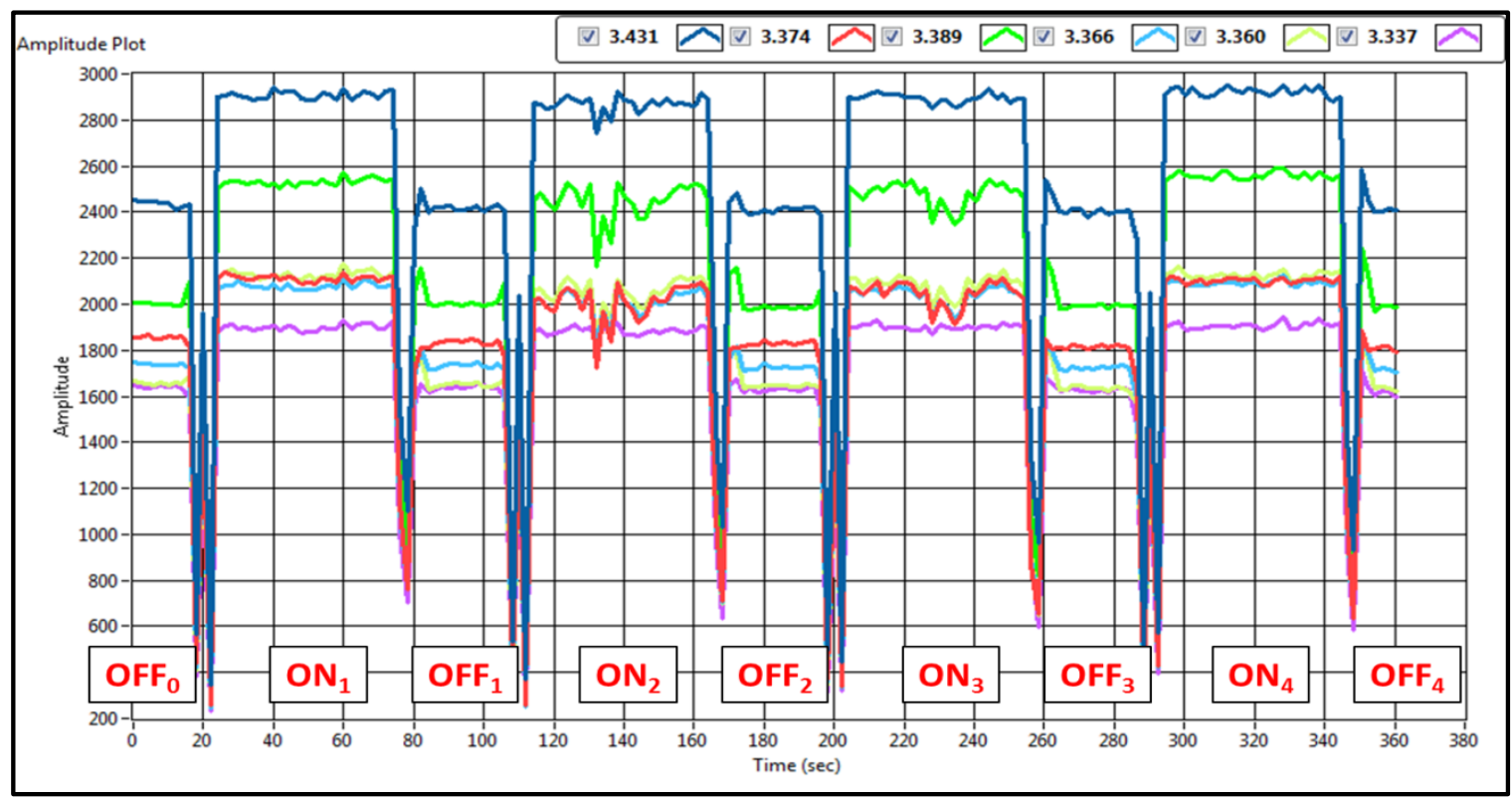

Figure 60: The spatial and temporal averaged amplitude for each of the sampled wavelengths.

Again, using the amplitude data to calculate the concentration path-length, it is clearly evident that there is gas present during the second and third On/Off sequence, and no gas during the first and fourth sequences.

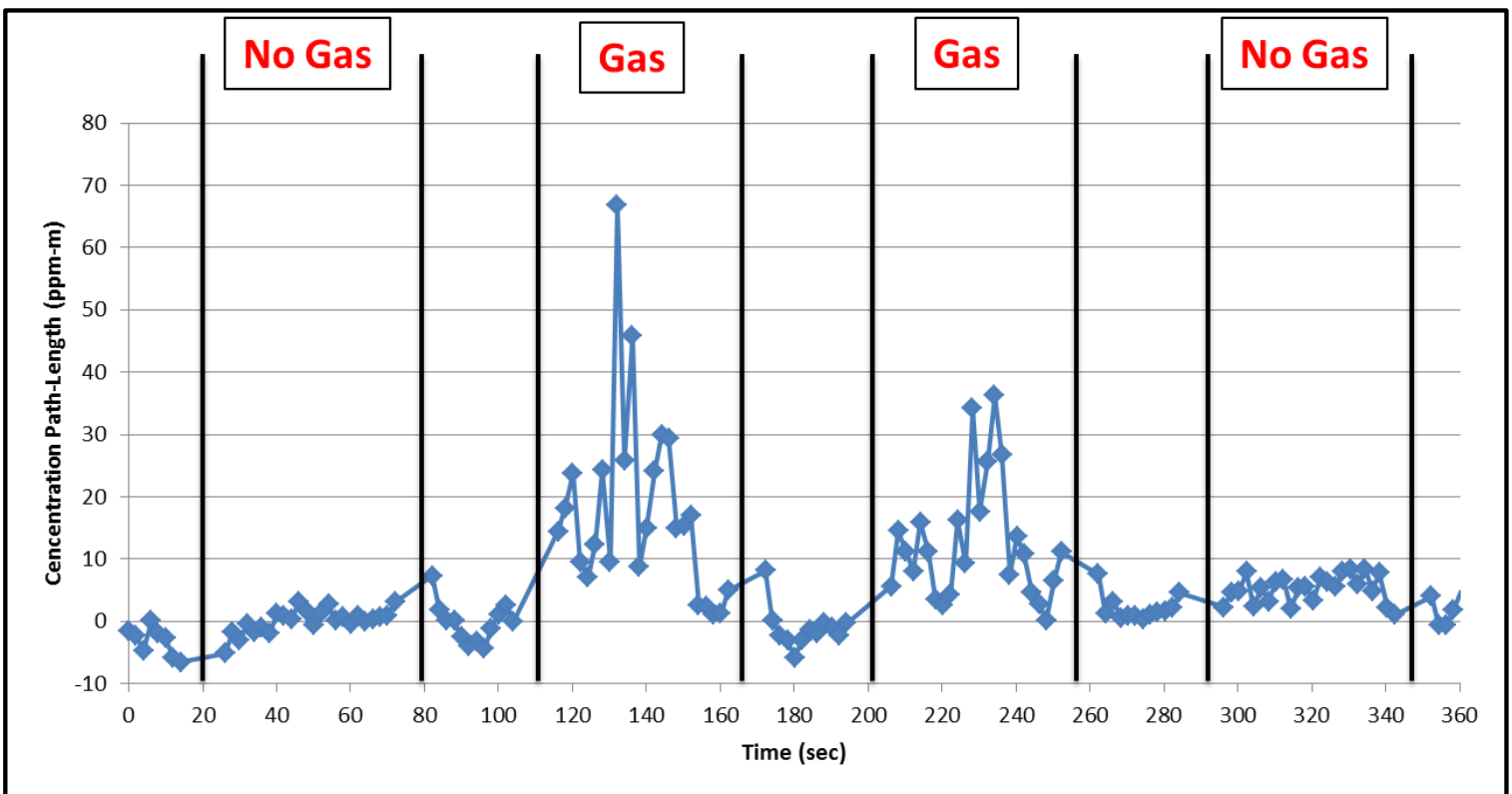

Figure 61: The concentration path-length calculation of butane for the second example. 


\section{Conclusion:}

The purpose of this project was to build a ground based remote sensing system that can detect, identify, and quantify a specific gaseous species in the 3.2-3.5 micron range using the DIAL technique. This thesis describes the approach used to develop and field an active remote sensing system. A brief description was given of what remote sensing is, and the technique used to analyze the data collected for a specific known material. The main components of the system were then discussed along with how the subsystems were characterized and controlled in order to produce the required wavelength from the transmitter. And finally, some results were discussed from collection in both a laboratory setting and a real-world setting. 


\section{Acknowledgement:}

I would like to thank my colleagues at Lawrence Livermore National Laboratory: Dr. Brent Stuart, Dr. June Yu, Dr. Christopher Ebbers, Dr. William Neuman, Mr. Vic Karpenko, Mr. Scott Mitchell, Mr. Rich Shuttlesworth, and Mr. Noel Peterson for their help throughout the project and with guidance on this thesis. 


\section{References:}

1. 1: R. E. Warren, "Detection and discrimination using multiple-wavelength differential absorption lidar," Applied Optics, Vol 24, p3541 (1985)

2. S.W. Sharpe, R.L. Sams, T.J. Johnson, P.M. Chu, G.C. Rhoderick and F.R. Guenther, "Creation of $0.10 \mathrm{~cm}-1$ Resolution, Quantitative, Infrared Spectral Libraries for Gas Samples", in Vibrational Spectroscopy-based Sensor Systems, Steven D. Christesen, Arthur J. Sedlacek III, Editors, SPIE Proceedings, 4577, 12-24, (2002).

3. T. J. Johnson, R. L. Sams and S. W. Sharpe, "The PNNL Quantitative Infrared Database for GasPhase Sensing: A Spectral Library for Environmental, Hazmat and Public Safety Standoff Detection," in Chemical and Biological Point Sensors for Homeland Defense, Arthur J. Sedlacek III, Steven D. Christesen, Richard Colton, Tuan Vo-Dinh, Editors, SPIE Proceedings, 5269, 159-167 (2004).

4. L. M. Little, "Pirelli Tuning Algorithm”, Unpublished Memo, (2008)

5. R. Paschotta, article on 'master oscillator power amplifier' in the Encyclopedia of Laser Physics and Technology, <https://www.rp-photonics.com/master_oscillator_power_amplifier.html>, accessed on 2016-04-29

6. D.H. Jundt, "Temperature-dependent Sellmeier equation for the index of refraction, ne, in congruent lithium niobate", Optics Letters, Vol. 22, No. 20, p 1553 (1997)

7. L. Goldberg and W. K. Burns, "Wide acceptance bandwidth difference frequency generation in quasi-phase-matched $\mathrm{LiNbO}_{3}{ }^{\prime \prime}$, Applied Physics Letters, 67, 2910 (1995)

8. A.V. Smith, SNLO nonlinear optics code avalilable from, AS-Photonics, Albuquerque, NM

9. F. Träger: Springer Handbook of Lasers and Optics (Springer, Berlin Heidelberg 2012) 